MULTISCALE PHASE CHANGE, HEAT AND MASS TRANSFER

IN DIRECT CONTACT MEMBRANE DISTILLATION

A Dissertation
Presented to
The Faculty of the Graduate School
at the University of Missouri-Columbia
In Partial Fulfilment
of the Requirement for the Degree
Doctor of Philosophy
BLNAZ NOROUZI
Dr. Chanwoo Park, Dissertation Supervisor

May 2020 
(C) Copyright by Elnaz Norouzi 2020

All Rights Reserved 
The undersigned, appointed by the dean of the Graduate School, have examined the dissertation entitled

\section{MULTISCALE PHASE CHANGE, HEAT AND MASS TRANSFER IN DIRECT CONTACT MEMBRANE DISTILLATION}

presented by Elnaz Norouzi, a candidate for the degree of Doctor of Philosophy in Mechanical Engineering and hereby certify that, in their opinion, it is worthy of acceptance.

Professor Chanwoo Park

Professor Jacob McFarland

Professor Gary L. Solbrekken

Professor Robert Tzou

Professor Yuwen Zhang

Professor Stephen Montgomery-Smith 
I dedicate my dissertation work to my family. A special feeling of gratitude to my loving parents, Nader and Touran whose words of encouragement and push for tenacity ring in my ears. My brothers Amir and Alireza have never left my side and are very special. I would not be the person I am today without you. 


\section{ACKNOWLEDGEMENT}

I would like to express my gratitude and appreciation for Professor Chanwoo Park. whose guidance, support and encouragement has been invaluable throughout this study. I also wish to thank all the members of my committee who have been a great source of support and guidance all these years. 


\section{TABLE OF CONTENTS}

ACKNOWLEDGEMENT ....................................................................................... ii

LIST OF FIGURES ...........................................................................................

LIST OF TABLES....................................................................................................... xii

ABSTRACT ....................................................................................................................................... xiii

NOMENCLATURE .............................................................................................................. $\mathrm{xV}$

CHAPTER 1: INTRODUCTION_............................................................................................ 1

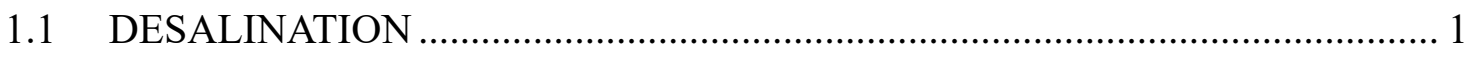

1.2 MULTISCALE HEAT AND MASS TRANSFER IN PHASE CHANGE

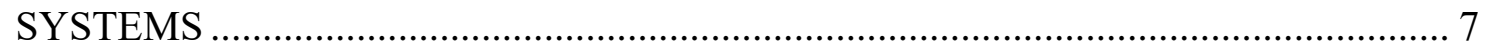

CHAPTER 2: DIRECT CONTACT MEMBRANE DISTILLATION USING DIESEL ENGINE WASTE HEAT ........................................................................................................ 12

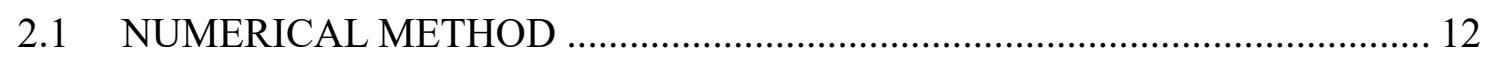

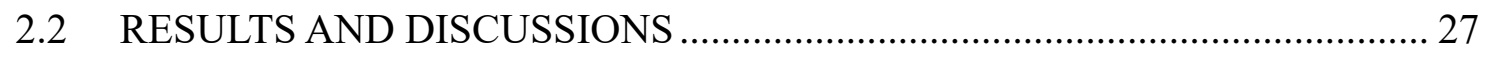

2.2.1 Effect of Membrane Length ................................................................. 28

2.2.2 Effect of Salt Concentration...................................................................... 32

2.2.3 Effect of Feed and Permeate Water Flow Rate ……………………............. 34

2.2.4 Effect of Feed and Permeate Inlet Temperature.......................................... 37

2.2.5 Parallel Arrangement of Multiple Direct Contact Membrane Distillation

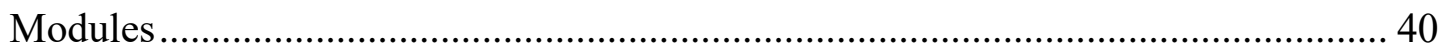

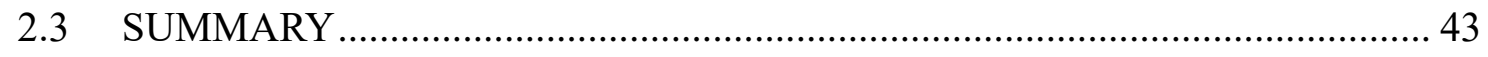




\section{CHAPTER 3: HEAT AND MASS TRANSFER IN DIRECT CONTACT}

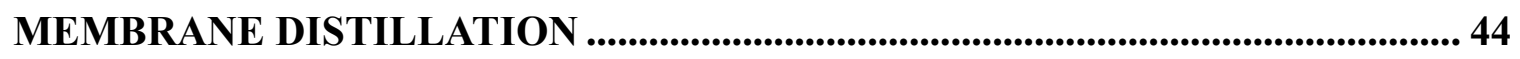

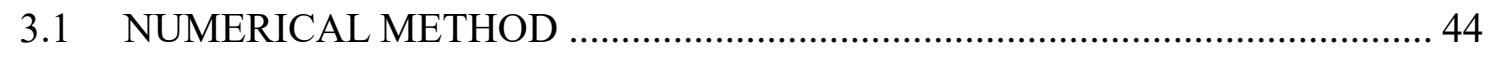

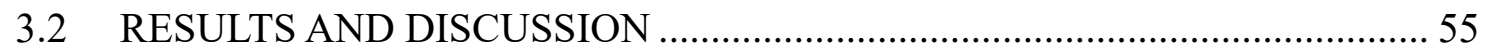

3.2.1 Baseline Results of Flow, Heat and Mass Transfers .................................... 56

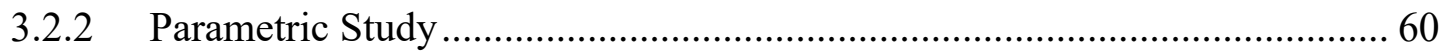

3.2.3 Effect of Mesh Screen Filament Spacing....................................................... 69

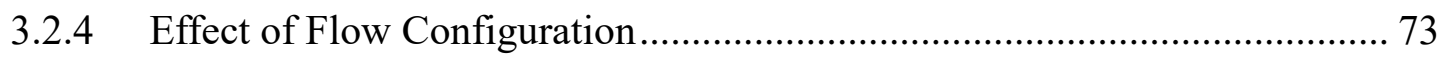

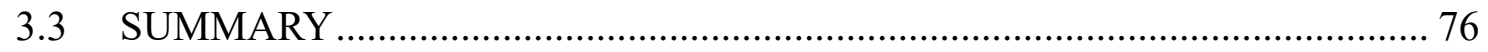

CHAPTER 4: SCALING EFFECT ON PERFORMANCE OF MEMBRANE DISTILLATION CRYSTALLIZATION FOR HYPERSALINE BRINES................ 77

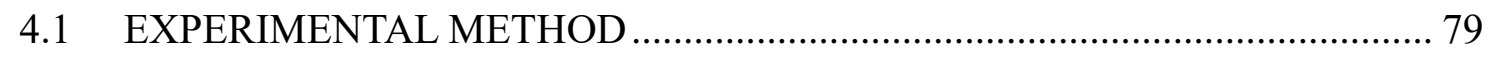

4.2 NUMERICAL AND ANALYTICAL ANALYSIS …………………………..... 81

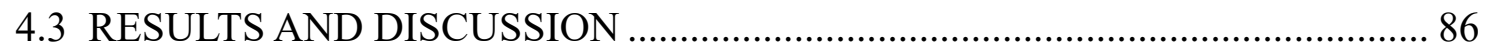

4.3.1 Effect of Salt Concentration..................................................................... 86

4.3.2 Effect of Inlet Feed and Permeate Temperatures ........................................ 87

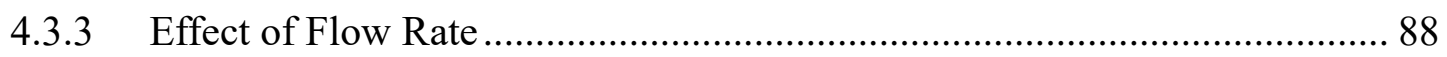

4.3.4 Membrane Distillation Scaling ................................................................ 89

4.3.5 Effects of Cooling Rates in Crystallization Process ……………................. 91

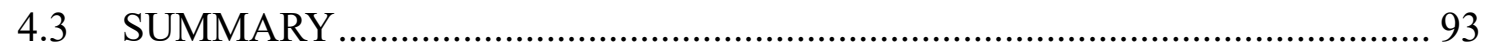

CHAPTER 5: CARBON NANOTUBE DIRECT CONTACT MEMBRANE DISTILLATION .................................................................................................................................... 95

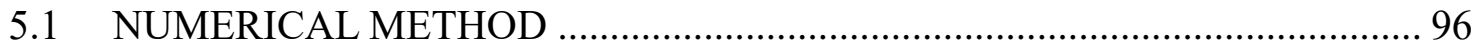




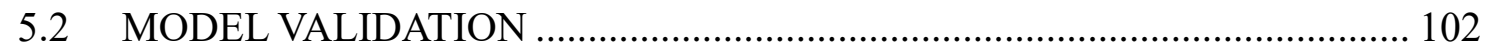

5.3 RESULTS AND DISCUSSION .......................................................... 105

5.3.1 Onset of Liquid Flooding and Contact Angle ....................................... 105

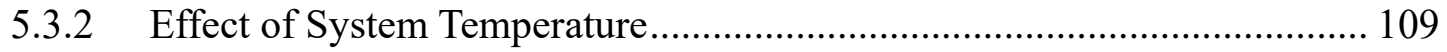

5.3.3 Effect of Reservoir Temperature Difference........................................... 111

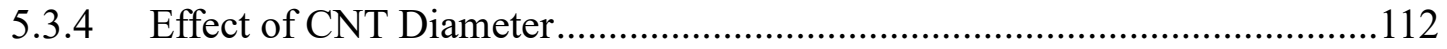

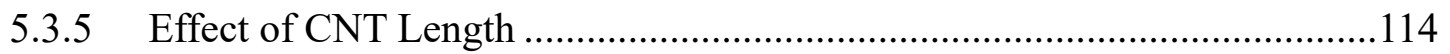

5.3.6 Effect of Interatomic Force Between CNT and Water...............................116

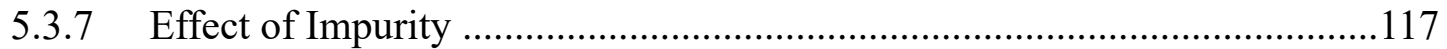

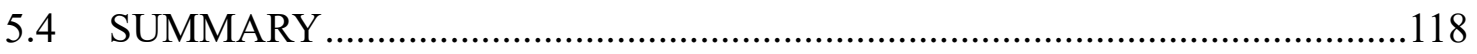

CHAPTER 6: CONCLUSIONS ..................................................................................... 120

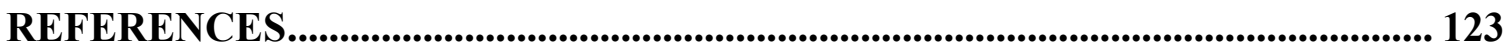

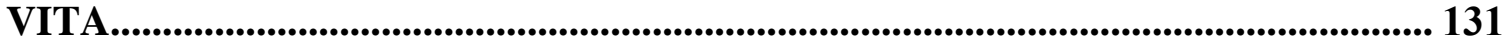




\section{LIST OF FIGURES}

Figure 1.1 Distribution of water in the world ......................................................... 1

Figure 1.2 Different types of water desalination technologies .................................... 3

Figure 1.3 Schematic representations of (a) DCMD, (b) AGMD, (c) SGMD, and (d)VMD

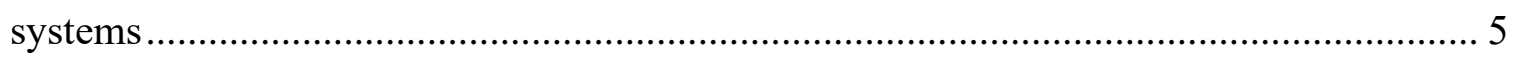

Figure 1.4 Schematic representations of Knudsen and molecular diffusions .................. 10

Figure 1.5 Schematic representation of crystal deposition ........................................11

Figure 2.1 (a). Schematic of a single-module DCMD system used for thermal-hydraulic network modeling. (b) Schematic of a multiple-module DCMD system connected in a

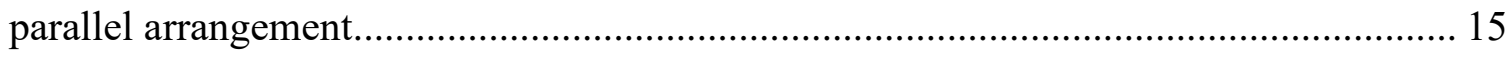

Figure 2.2 Mechanism of mass transport of the membrane distillation with pore size

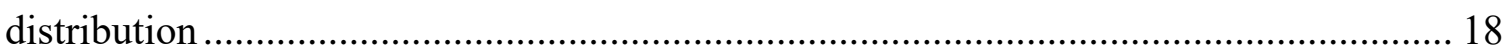

Figure 2.3 Variations of feed and permeate fluid temperatures in a single-module DCMD system and the vapor pressure difference across the membrane with different membrane length under the baseline conditions

Figure 2.4 (a) Variation of mass flux of water distillate production and (b) conduction and phase change heat fluxes in a single-module DCMD system with different membrane length under the baseline conditions 30

Figure 2.5 Effects of membrane module length on (a) heat transfer rates and thermal efficiency and (b) total water distillate production rate in a single-module DCMD system

Figure 2.6 (a) Variation of mass flux of water production with different inlet feed concentration and the profile of $\mathrm{NaCl}$ concentration along the $\mathrm{MD}$ channel under the 
baseline conditions. (b) Effect of inlet feed concentration on thermal efficiency and total water production rate in a single-module DCMD system 33

Figure 2.7 (a) Effect of feed and permeate mass flow rates on the temperature profiles and (b) the mass flux of the water distillate production of a single-module DCMD system .. 35 Figure 2.8 Effects of feed and permeate mass flow rates on (a) the heat transfer rates and thermal efficiency and (b) total water distillate production of a single-module DCMD system 37

Figure 2.9 Effect of feed inlet temperature on the mass flux of the water distillate production of a single-module DCMD system 38

Figure 2.10 Variations of total water distillate production and thermal efficiency of a singlemodule DCMD system with varying inlet temperature difference of feed and permeate streams 40

Figure 2.11 (a) Variations of heat transfer area of the heat source heat exchanger (HX1), number of membrane modules and total membrane area of a multiple-module DCMD system with varying membrane module length. (b) Variations of total water distillate production, mass flux, and thermal efficiency and effectiveness of the heat source heat exchanger (HX1) of a multiple-module DCMD system with varying membrane module length 42

Figure 3.1 (a) Computational domain, (b) geometries and dimensions of the filaments of the mesh screen spacer and the spacing between the filaments of the DCMD system and (c) boundary conditions used for CFD analysis

Figure 3.2 Thermal circuit diagram for heat transfers in the DCMD system 54 
Figure 3.3 (a) velocity contour in the feed and permeate channels, (b) temperature contour in the feed and permeate channels, (c) variations of fluid temperatures of the feed and permeate channels and conduction and phase change heat fluxes, and (d) variations of the vapor pressures of the feed and permeate channels and vapor pressure difference between the feed and permeate channels and membrane mass flux 59

Figure 3.4 Effect of feed temperature on (a) water production rate and outlet fluid temperature and (b) conduction and phase change heat transfer rates and thermal efficiency of the DCMD system 62

Figure 3.5 Effect of permeate temperature on (a) water production rate and outlet fluid temperature and (b) conduction and phase change heat transfer rates and thermal efficiency of the DCMD system 64

Figure 3.6 Effect of mass flow rates of the feed and permeate channels on (a) water production rate and outlet fluid temperature and (b) heat transfer rates and thermal efficiency of a DCMD system 66 Figure 3.7 (a) Effect of $\mathrm{NaCl}$ concentration in the feed channel on water production rate. (b) Variation of $\mathrm{NaCl}$ concentration on the membrane surface and saturation pressure in the feed channel and (c) contour of the $\mathrm{NaCl}$ concentration in the feed channel for a salt concentration of $C_{f, i}=0.0457 \mathrm{~kg}-\mathrm{NaCl} / \mathrm{kg}$ 68 Figure 3.8 Effect of non-spacer and spacer-filled channels with mesh screen filament spacings of $10 \mathrm{~mm}$ and $5 \mathrm{~mm}$ on (a) thermal resistances including convection thermal resistance in the feed (red lines) and permeate (blue lines) streams, combination of conduction and phase change thermal resistances (green lines) and total thermal resistance (black lines) (b) membrane surface temperatures in the feed (red lines) and permeate (blue viii 
lines) channels and conduction (green lines) and phase change (black lines) heat fluxes and (c) mass fluxes and differences of saturation pressure between the feed and permeate channels 72

Figure 3.9 Variations of temperature and concentration polarization coefficients for nonspacer and spacer-filled channels with mesh screen filament spacings of $10 \mathrm{~mm}$ and $5 \mathrm{~mm}$. The concentration polarization coefficients are based on a salt concentration of $C_{f, i}=0.0457$

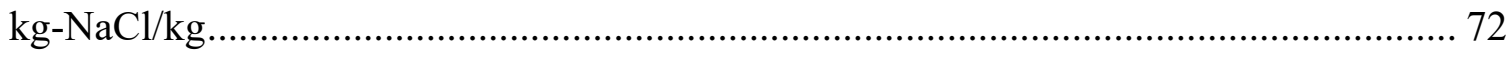

Figure 3.10 Variations of (a) thermal resistances for counter and parallel flows including convection thermal resistance in the feed (red lines) and permeate (blue lines) streams, combination of conduction and phase change thermal resistances (green lines) and total thermal resistance (black lines) (b) membrane surface temperatures in the feed (red solid line) and permeate (blue solid line) channels, temperature difference between feed and permeate streams (black solid line) and conduction (green solid line) and phase change (black dashed line) heat fluxes and (c) vapor pressures (red and blue solid lines), differences of vapor pressure between the feed and permeate channels (black dashed line) and mass flux of the membrane module (black solid line) for parallel flow arrangement and

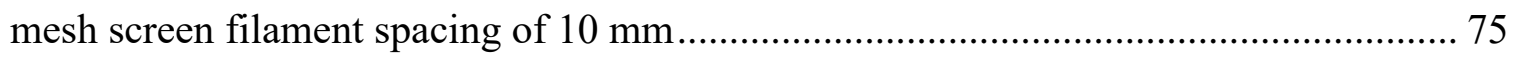

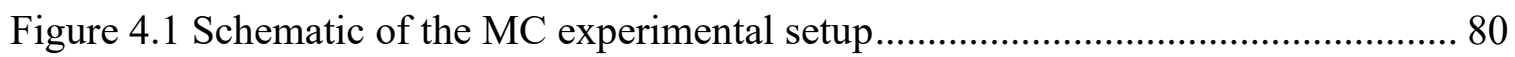

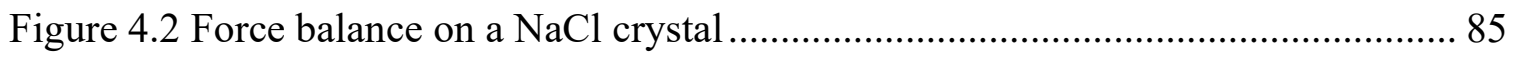

Figure 4.3 Effect of feed water concentration on water flux, outlet feed, and permeate water temperatures. 87

Figure 4.4 Effect of inlet feed temperature on water flux, outlet feed, and permeate temperatures 88 
Figure 4.5 Effect of inlet permeate temperature on water flux, outlet feed and permeate temperatures 88

Figure 4.6 Effects of feed and permeate flow rates on water flux, outlet feed and permeate temperatures. 89

Figure 4.7 Water flux and saturation ratio versus time in MD operation 91

Figure 4.8 Brine temperature profiles in the crystallization process 92 Figure 4.9 Microscopic images of $\mathrm{NaCl}$ crystals for natural and forced cooling methods 93

Figure 4.10 Distribution of salt crystal sizes for forced and natural cooling. 93

Figure 5.1 CNT membrane (a) before and (b) after flooding condition, and (c) CNT DCMD system showing water vapor flow between the hot and cold reserviors 100 Figure 5.2 (a) Pressure profile across the CNT membrane (not scaled). (b) Comparison of the mass diffusivities from the MD simulation and experimental measurement [64] ... 104 Figure 5.3 Effect of different incremental movement on showing water meniscus rupture 107

Figure 5.4 (a) Isochoric profile and curve fitting of $5.0 \mathrm{~nm} \mathrm{CNT} \mathrm{diameter.} \mathrm{(b)} \mathrm{Variation} \mathrm{of}$ contact angle with respect to CNT diameter. 108

Figure 5.5 Variation of contact angle with respect to CNT diameter 108 Figure 5.6 Variations of water vapor flux, diffusivity, and TMAC with respect to system temperature 111

Figure 5.7 Effect of reservoir temperature difference on water vapor flux, diffusivity, and TMAC .112 
Figure 5.8 Variations of water vapor flux, diffusivity, TMAC, and collision residence time

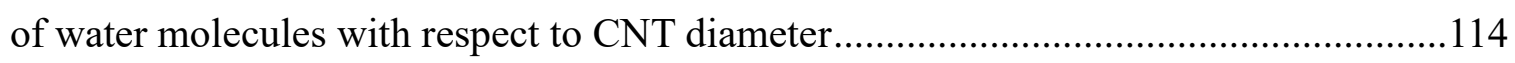

Figure 5.9 Effect of CNT length on water vapor flux, mass diffusivities, and TMAC ...115 Figure 5.10 Variations of water vapor flux, diffusivity, TMAC and residence time with respect to energy constant of LJ potential between water molecules and carbon atoms .117 Figure 5.11 Effect of Sodium Chloride concentration on water vapor flux, diffusivity and

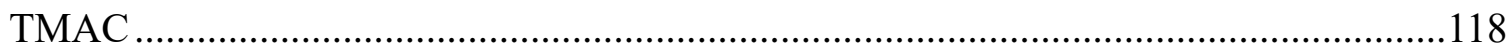




\section{LIST OF TABLES}

Table 2.1 Dimensional and thermophysical properties of membrane and baseline operating conditions and dimension of Direct Contact Membrane Distillation system .................. 22

Table 2.2 Dimensions and thermophysical property of the flat-plate heat exchanger (HX1)

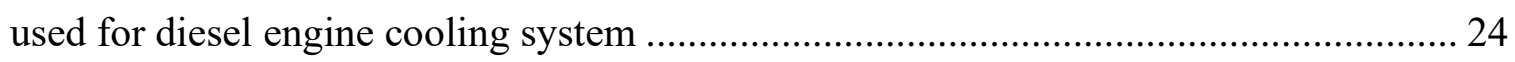

Table 3.1 Dimensional and thermophysical properties of the membrane used in the DCMD

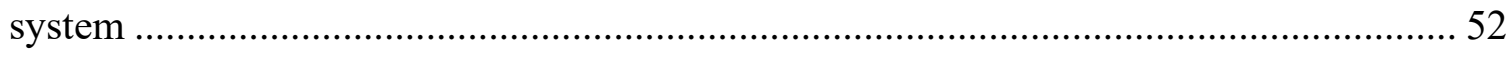

Table 4.1 Dimensional and thermophysical properties of the membrane used in the DCMD

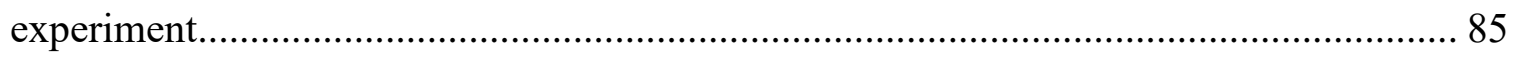

Table 4.2 Constant parameters used in Eq. (4.18) ........................................................ 85

Table 5.1 The Lennard-Jones parameters and charges of the simulated atoms ................ 98

Table 5.2 NEMD simulation conditions and dimensions ............................................. 109 


\begin{abstract}
The global water shortage has become a serious threat for the world and the most promising solution for the water issue is the desalination of seawater or brackish water. In this work, direct contact membrane distillation (DCMD) as one of thermal desalination technologies was numerically and exprimentally analyzed to study its performance.

A large DCMD system with multiple membrane modules in a parallel arrangement running on the waste heat from a diesel power generator was numerically analyzed using a thermo-fluid network model to study the technical feasibility of the use of the low-grade engine waste heat and simulate the distillation performance of the DCMD system. Next, a small DCMD experimental apparatus was fabricated to test for the distillation performance for various operating conditions (inlet temperatures, flow rates of feed and permeate streams and $\mathrm{NaCl}$ concentration) and design variables (filament spacing of a screen spacer in the flow channels and flow configuration).
\end{abstract}

In the DCMD, two different regimes were observed in the water flux behavior regarding the salinity of feed water. In the first regime, from low $\mathrm{NaCl}$ concentration to $90 \%$ saturated $\mathrm{NaCl}$ concentration, there was a gradual decrease in the water flux due to the suppression of vapor pressure at the feed water which is simulated by a CFD model. In the second regime, at higher $90 \%$ saturated $\mathrm{NaCl}$ concentration, there was a sharp drop in the water flux due to the deposition of $\mathrm{NaCl}$ crystals on the membrane surface which is simulated by an analytical model using the adjusting parameter from the experiment.

Finally, a nanoscale DCMD using Carbon Nanotube (CNT) membrane was numerically analyzed using non-equilibrium molecular dynamics (NEMD) simulation for different diameters and lengths of the CNT and operating conditions such as system xiii 
temperature, temperature difference between the feed (hot) and permeate (cold) reservoirs, and sodium chloride $(\mathrm{NaCl})$ concentration in the feed reservoir. The distillation performance of the DCMD systems is enhanced by increasing system temperature, temperature difference between feed and permeate streams, and decreasing the $\mathrm{NaCl}$ concentration. The permeability of the CNT membrane $\left(1.8 \times 10^{-5} \mathrm{liter} / \mathrm{m}^{2}-\mathrm{s}-\mathrm{Pa}\right)$ was found two orders-of-magnitudes higher than a Polytetrafluoroethylene (PTFE) membrane $(1.7 \times$ $10^{-7} \mathrm{liter} / \mathrm{m}^{2}$-s-Pa ) used in our experimental work. 


\section{NOMENCLATURE}

\begin{tabular}{|c|c|}
\hline$A$ & area $\left[\mathrm{m}^{2}\right]$ \\
\hline$a$ & thermal accommodation coefficient, open surface $\left[\mathrm{m}^{2}\right]$ \\
\hline$a_{p}$ & channel height of heat exchanger $[\mathrm{m}]$ \\
\hline$a_{w}$ & water activity [-] \\
\hline$C$ & heat capacity $C=\dot{m} c\left[\mathrm{~W} \mathrm{~K}^{-1}\right]$, concentration $\left[\mathrm{gr} \mathrm{L}^{-1}\right]$ \\
\hline$C_{A}$ & system dependent constant [N.m] \\
\hline$C_{L}$ & lubrication constant [Pa.s] \\
\hline$C_{m}$ & coefficient of deposited mass [-] \\
\hline$C_{T}$ & stress coefficient of tangential force $[\mathrm{Pa}]$ \\
\hline$C_{1}$ & integration constant $[1 / \mathrm{Pa}]$ \\
\hline$C_{2}$ & integration constant $\left[\mathrm{m}^{2}\right]$ \\
\hline$c$ & specific heat capacity $\left[\mathrm{J} \mathrm{kg}^{-1} \mathrm{~K}^{-1}\right]$ \\
\hline$c_{v}$ & specific heat capacity at constant volume $\left[\mathrm{J}\right.$ mole $\left.{ }^{-1} \mathrm{~K}^{-1}\right]$ \\
\hline$D$ & diffusivity $\left[\mathrm{m}^{2} \mathrm{~s}^{-1}\right]$ \\
\hline$D_{h}$ & hydraulic diameter $[\mathrm{m}]$ \\
\hline$D_{v}$ & turbulent destruction $\left[\mathrm{kg} \mathrm{m}^{-1} \mathrm{~s}^{-2}\right]$ \\
\hline$D_{K n}$ & Knudsen diffusion coefficient $\left[\mathrm{m}^{2} \mathrm{~s}^{-1}\right]$ \\
\hline$D_{w v-a}$ & diffusivity of water vapor-air mixture $\left[\mathrm{m}^{2} \mathrm{~s}^{-1}\right]$ \\
\hline$d$ & diameter $[\mathrm{m}]$ \\
\hline$e$ & roughness $[\mathrm{m}]$ \\
\hline $\mathbf{F}$ & interaction force $[\mathrm{N}]$ \\
\hline
\end{tabular}


$f \quad$ tangential momentum accommodation coefficient

$f_{v 1} \quad$ viscous damping function

$G_{v} \quad$ turbulent production $\left[\mathrm{kg} \mathrm{m}^{-1} \mathrm{~s}^{-2}\right]$

$H \quad$ height $[\mathrm{m}]$

$h \quad$ heat transfer coefficient $\left[\mathrm{W} \mathrm{m}{ }^{-2} \mathrm{~K}^{-1}\right]$

$h_{f g} \quad$ latent heat $\left[\mathrm{J} \mathrm{kg}^{-1}\right]$

$J \quad$ mass flux $\left[\mathrm{kg} \mathrm{m}^{-2} \mathrm{~s}^{-1}\right]$, water vapor flux $\left[\mathrm{nm}^{-2} \mathrm{~ns}^{-1}\right]$

$k \quad$ thermal conductivity $\left[\mathrm{W} \mathrm{m}^{-1} \mathrm{~K}^{-1}\right]$

$k_{\mathrm{B}} \quad$ Boltzmann constant $\left[\mathrm{J} \mathrm{K}^{-1}\right]$

$L \quad$ length [m or $\AA$ ]

$M \quad$ molecular weight $\left[\mathrm{kg} \mathrm{mol}^{-1}\right.$ or $\left.\mathrm{g} \mathrm{mol}^{-1}\right]$, mole fraction [-]

$m \quad$ mass $[\mathrm{kg}]$

$\dot{m} \quad$ mass flow rate $\left[\mathrm{kg} \mathrm{s}^{-1}\right]$

$N$ number of flow channels of heat exchanger, number of membrane module, number of atoms

$N_{\mathrm{A}} \quad$ Avogadro number

$\mathrm{Nu} \quad$ Nusselt number

NTU number of transfer unit

$n \quad$ number density [atoms $\mathrm{nm}^{-3}$ ]

$\dot{n} \quad$ number flow rate [atoms $\mathrm{ns}^{-1}$ ]

$p \quad$ pressure $[\mathrm{Pa}]$

Pr Prandtl number 


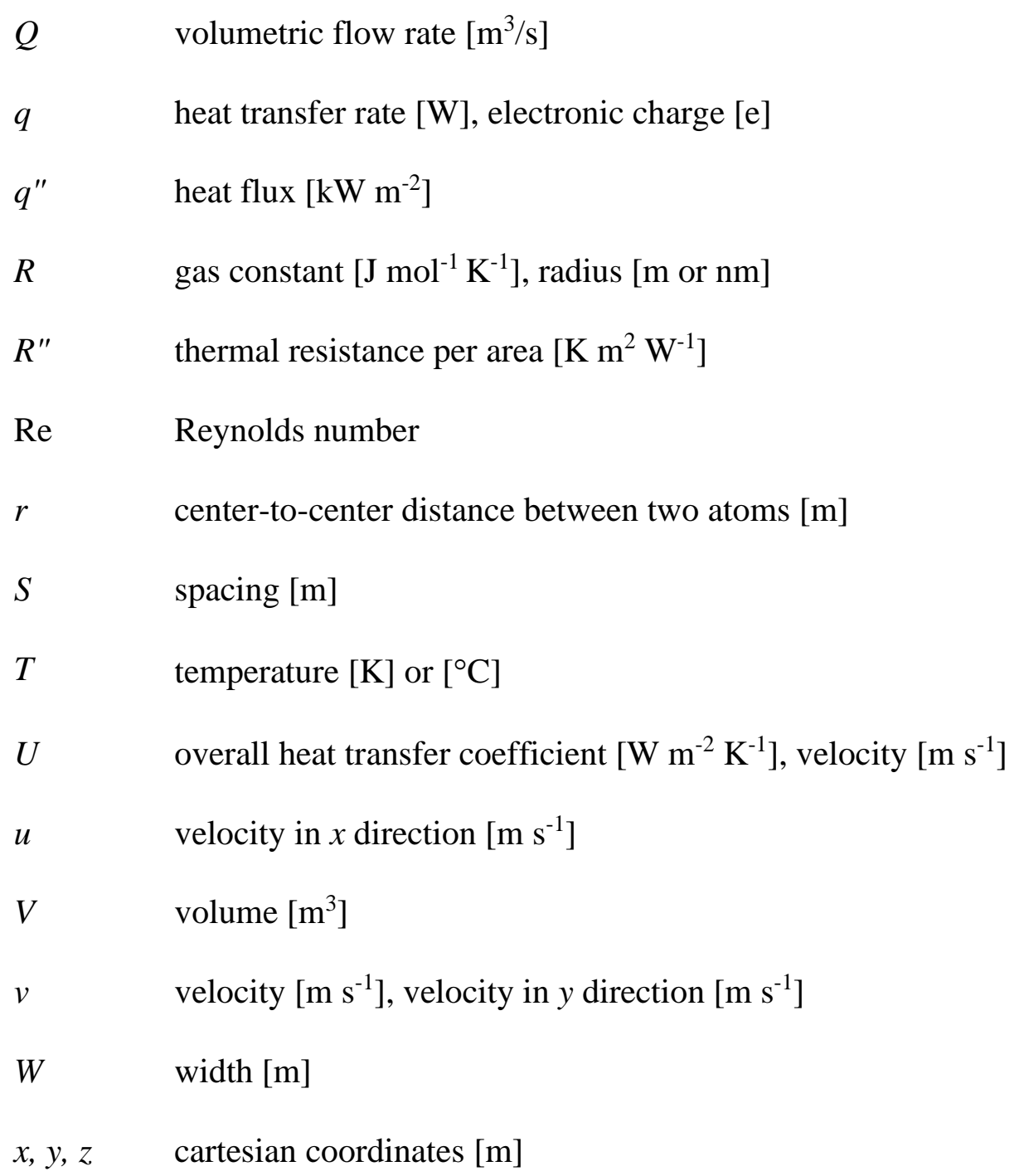

\section{Greek letters}

$\alpha \quad$ ratio of water molecular weight to air molecular weight

$\gamma \quad$ surface tension $[\mathrm{N} / \mathrm{m}]$

$\delta \quad$ thickness [m]

$\varepsilon \quad$ porosity, effectiveness, Lennard-Jones potential energy constant [ $\left.\mathrm{kcal} \mathrm{mole}^{-1}\right]$

$\eta \quad$ efficiency, friction coefficient 


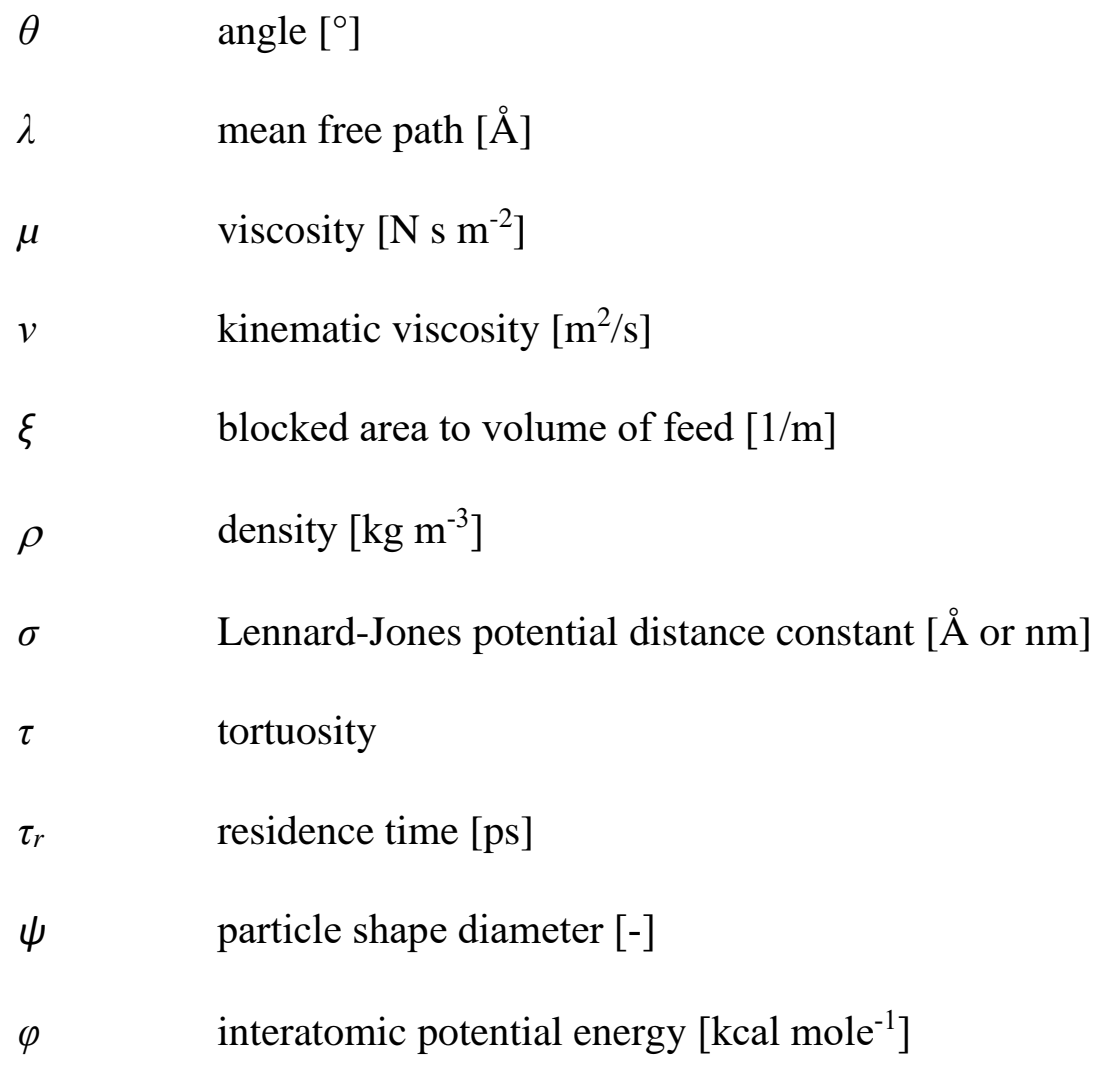

\section{Subscripts}

$\begin{array}{ll}\text { A } & \text { Avogadro } \\ a & \text { air } \\ \text { ad } & \text { adsorbed } \\ \text { amb } & \text { ambient } \\ \text { Ar } & \text { Argon } \\ \text { ave } & \text { average } \\ \text { B } & \text { Boltzmann } \\ b & \text { brine } \\ \text { C } & \text { carbon }\end{array}$




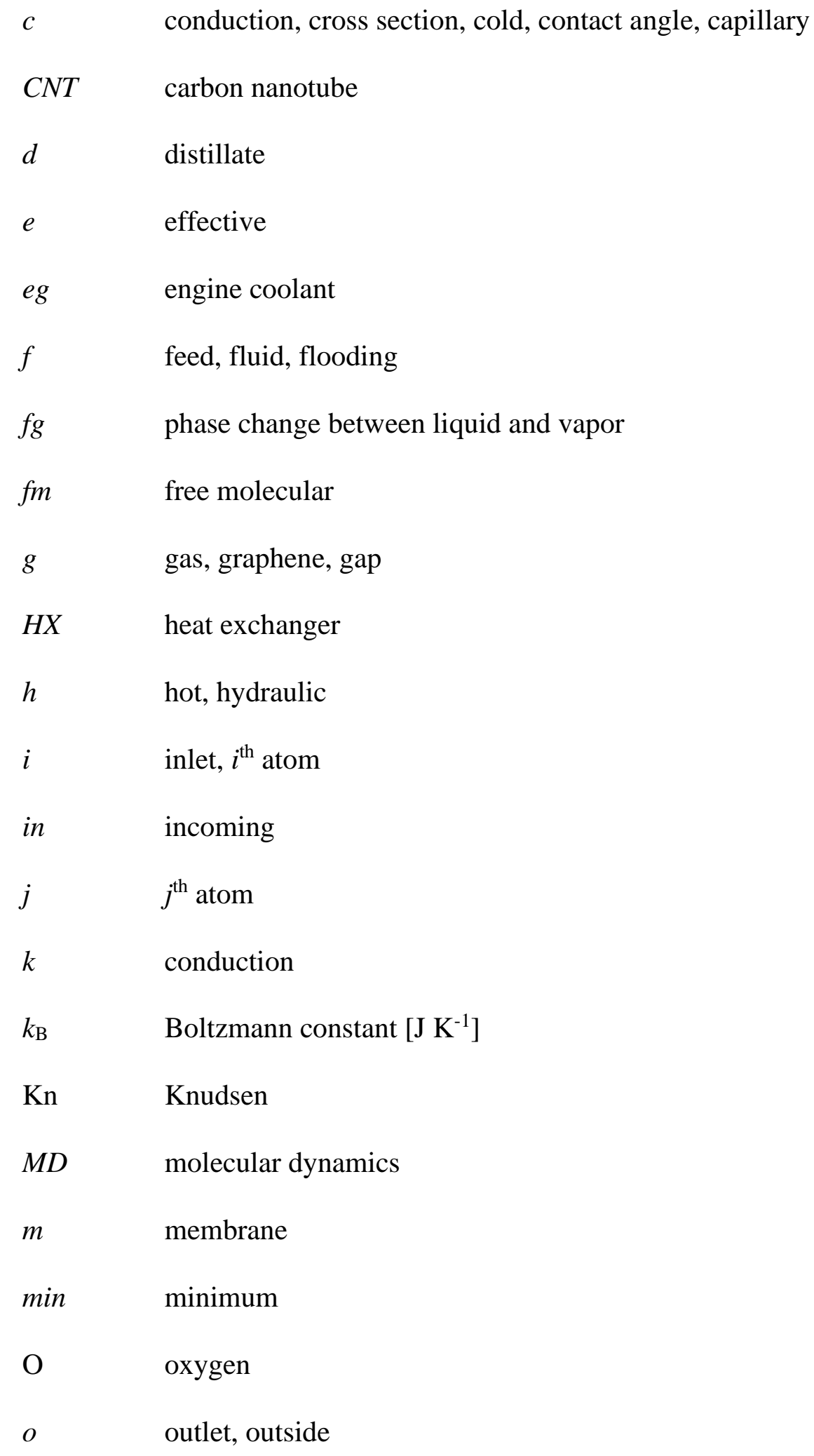




$\begin{array}{ll}\text { out } & \text { outgoing } \\ p & \text { permeate, pore, plate, piston, post } \\ \mathrm{Pt} & \text { platinum } \\ r & \text { ratio } \\ \text { Sm } & \text { Smoluchowski } \\ s & \text { solid, salt } \\ s a t & \text { saturation } \\ T & \text { total } \\ t & \text { thermal, transport, tangential, total, turbulent } \\ v & \text { vapor, volumetric } \\ w & \text { water } \\ w v & \text { water vapor } \\ x, y, z & \text { cartesian coordinate variables }\end{array}$




\section{CHAPTER 1: INTRODUCTION}

\subsection{DESALINATION}

Each day humans must consume a certain amount of water to survive. While nearly $70 \%$ of the world is covered by water, only $2.5 \%$ is fresh and suitable for drinking. On the other hand, much of this freshwater is trapped in glaciers and snowfields, and less than $1 \%$ is readily accessible for direct human use (Figure 1.1). During the last century, water usage has increased at twice the rate of population increase. The United Nation estimates that in less than 25 years, if present water consumption trends continue, 5 billion people will be living in areas where it will be impossible or difficult to meet basic water needs for sanitation, cooking and drinking. With consideration of no alternate resource for water, a solution for the water shortage crisis is desalination water from the ocean and sea. Nowadays, this solution is the easiest way to provide water demand for human beings.

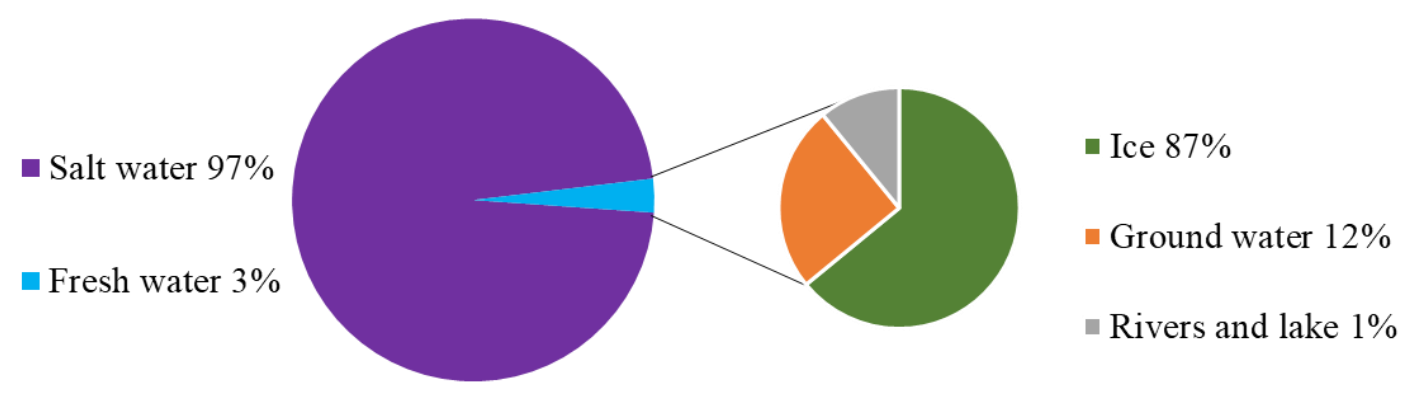

\section{Figure 1.1 Distribution of water in the world}

Desalination technologies are classified into two different types, thermal process technologies and mechanical or electrical technologies as shown in Fig 1.2. In the first type, thermal process technologies, the heated feed water is allowed to evaporate in the 
evaporator to produce water vapor which is condensed in a condenser to produce distillate. In the second type of desalination, fresh water is produced from saline water by allowing water molecules (reverse osmosis) or ions (electro-dialysis) to pass through membranes by applying s high pressure above osmotic pressure or an electric potential. The most common thermal desalination systems are humidification and dehumidification $(\mathrm{HDH})$, multi-stage flash (MSF), multi-effect distillation (MED), vapor compressor desalination (VCD). The main concept of $\mathrm{HDH}$ is that a higher temperature of the air has a higher capacity of the moisture. For the HDH system, a large number of stages is needed to achieve a large water production and therefore, results in a large capital cost. The efficiency of the HDH can be improved by recapturing the enthalpy of vaporization, separating the evaporation and condensation processes, and incorporating regenerative heating of the feed water stream in the condenser [1]. In the MSF system, the feed saline water is heated above the saturation temperature and is made flash in the vessel where low pressure is maintained. MED consists of vessels maintained successively at low pressure where saline water is sprayed in. The heat required to cause evaporation in the first vessel can be supplied by solar energy and the latent heat of the produced vapors in the previous vessels are successfully utilized for the next vessel in MED. In VCD, hot saline water is flashed into low-pressure vessels and the vapors are compressed with a compressor to raise condensation pressure and temperature. Another way to separate salt from water is to solidify salt in the saline water in freeze desalination systems. The saline water is cooled below its freezing point, and the ice crystals of pure water are formed on the surface. 


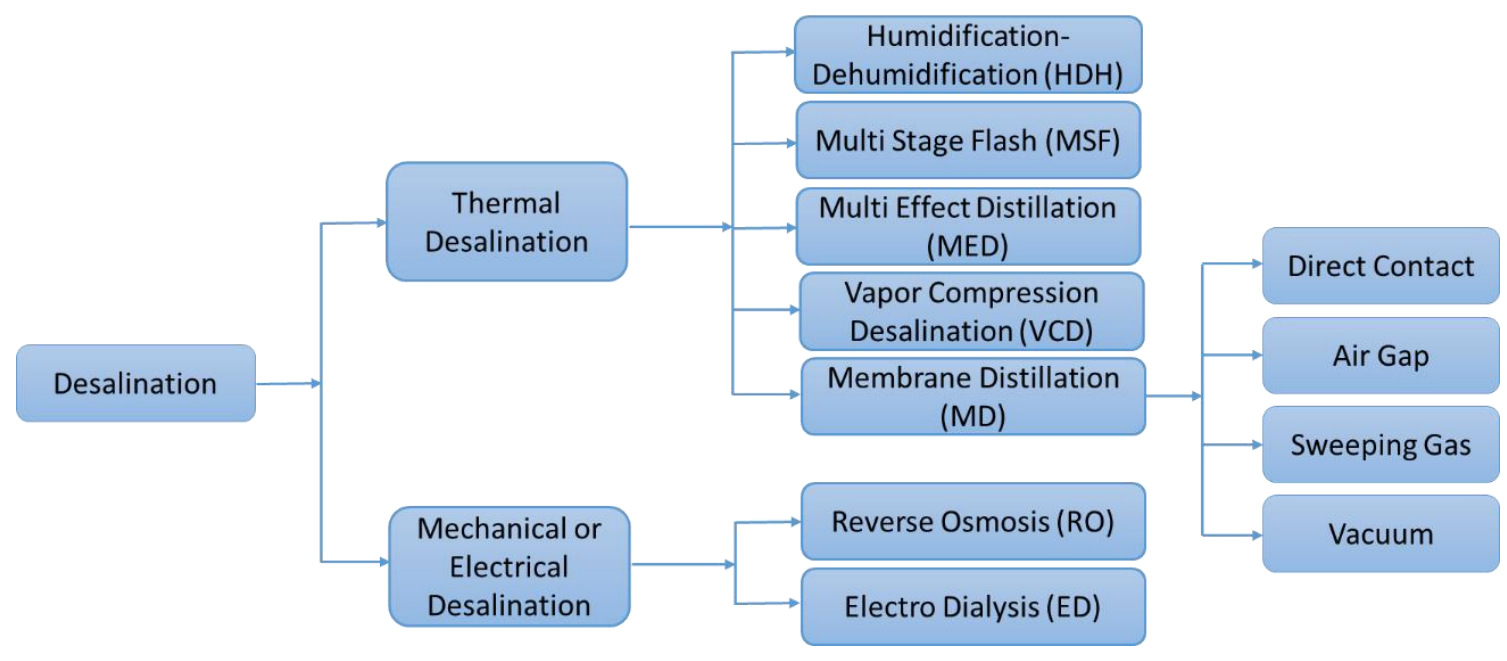

Figure 1.2 Different types of water desalination technologies

In water distillation technologies, membrane-based technologies have been growing in the last decades. Most of the membrane transport processes are working on an osmotic pressure, an electric potential or a chemical potential at a uniform temperature such as reverse osmosis membrane and electro dialysis. However, membrane distillation is running on the partial pressure gradient due to non-isothermal process through a membrane which is porous. In fact, membrane distillation (MD) is a water distillation technology using liquid/vapor phase changes (evaporation and condensation) in which only water vapor molecules are transported through a micro/nano-porous hydrophobic membrane driven by the vapor pressure difference created between hot (feed) and cold (permeate) fluid streams. The pore sizes are ranging from several nanometers to few micrometers. Currently, micro/nano-porous membrane distillation is made of different polymers as polypropylene (PP), polyvinylidene fluoride (PVDF), polytetrafluoroethylene (PTFE) and polyethylene (PE). To improve the productivity of the membrane distillation (MD), the design of the membrane distillation can be modified with micro- and 
nanostructures morphologies [2, 3]. The MD represents one of the most recent developments in thermal distillation processes despite the fact that the technology has been around since the late 60's. Recently, interest in MD has been aroused due to the environmental concerns and rising energy prices. Nowadays, MD has become costcompetitive with conventional distillation technologies.

There are four different membrane distillation configurations including direct contact, air gap, sweeping air gap, and vacuum membrane. In Direct Contact Membrane Distillation (DCMD), feed hot water is in direct contact with membrane, and therefore, evaporation occurs at the entrance of the membrane pores [Fig. 1.3(a)]. The generated vapor is driven to the other side of membrane by saturation vapor difference, since permeate stream with a lower temperature exists at the other side of membrane. Due to the high hydrophobicity of the membrane (contact angle $120^{\circ}$ ), liquid water cannot penetrate to the membrane pores, only water vapor transport from feed water to the permeate side. Due to the simplicity of the DCMD system, it is used extensively. The drawback of this system is a high heat loss through the membrane by conduction heat transfer. In Air Gap Membrane Distillation (AGMD), feed water has direct contact with the membrane, while there is stagnant air between the permeate side of the membrane and the condensation surface for the permeate vapor [Fig. 1.3(b)]. The stagnant air in the system conveys both advantage and disadvantage for the distillation performance. The main advantage is the reduction of heat loss by conduction heat transfer since air thermal conductivity is lower than water. The disadvantage of AGMD is that there is an additional mass resistance for water vapor transport due to the air gap. In sweeping gas membrane distillation (SGMD), non-condensable gas such as air is used to sweep the vapor on the permeate membrane side 
to condense outside the membrane module [Fig. 1.3(c)]. The gas flow, like in AGMD, reduces the conduction heat loss across the membrane and enhances the mass diffusion of water vapor. The main disadvantage of this configuration is that a small volume of permeate diffuses in a large sweep gas volume, requiring a large condenser because of a low partial pressure for water vapor. In the vacuum membrane distillation (VMD) configuration, a vacuum pump is used to create a vacuum on the permeate membrane side [Fig. 1.3(d)] and condensation takes place outside the membrane module $[4,5]$. DCMD remains a good choice because of its lower mass resistance and simple design, save for its disadvantage of a higher heat conduction loss.

(a)

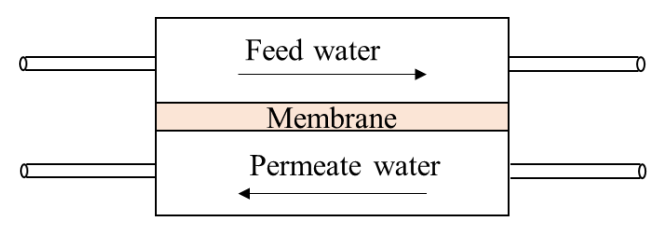

(c)

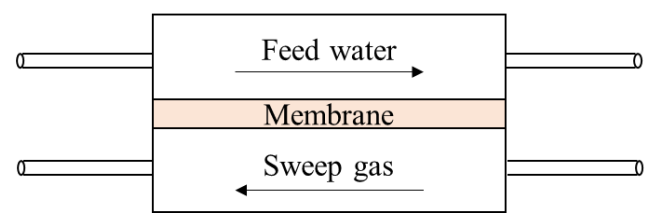

(b)

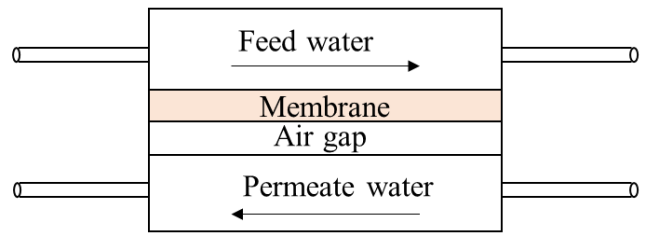

(d)

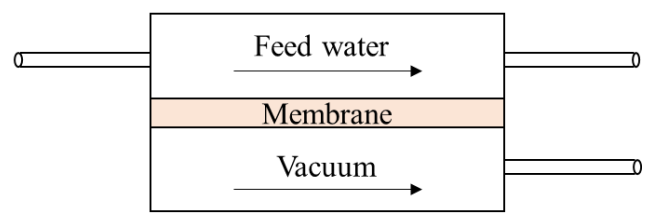

Figure 1.3 Schematic representations of (a) DCMD, (b) AGMD, (c) SGMD, and (d)VMD systems

Powering MD systems using renewable and waste heat has shown much promise, demonstrated by the work of several researchers [6-11]. Saffarini et al. performed an economic evaluation of solar-powered DCMD systems and found that the most economic configuration is a DCMD system coupled with a heat exchanger compared to AGMD and VMD [8]. Suarez et al. performed experiments on a DCMD system driven by a Salinity 
Gradient Solar Pond system to measure its water production rates and associated energy consumption [10]. Sarbatly and Chiam evaluated the combination of geothermal energy with VMD, and showed that geothermal energy could reduce the total energy consumption by approximately $95 \%$ and cost by at least $\$ 0.72 / \mathrm{m}^{3}[9]$.

The MD suffers from low water flux and researchers are searching to find a suitable material for the better water flux performance. Carbon Nanotubes (CNTs) with the unique nature of the one-dimensional structure, exceptional electrical, mechanical and thermal characteristics have inspired many researchers to use in the membrane distillation. Recently, Gethard et al. [12] showed that for a membrane distillation, adding CNT to polyvinylidene fluoride (PVDF) is effective to enhance water flux by $85 \%$. They postulated that the increase in the water flux might be attributed to the high capacity of diffusion transport of water vapor via the smooth surface [13] and the hydrophobic nature of CNT [14]. Most recently, An et al. illustrated that adding CNT to polyvinylidene fluoridehexafluoropropylene (PVDF-HFP) membrane results in more than $100 \%$ improvement in water flux of membrane distillation [15]. This improvement was explained by CNT surface diffusion. Furthermore, buckypaper membrane is a CNT-based membrane, and was implemented in a direct contact membrane distillation with $99 \%$ salt rejection $[16,17]$. 


\subsection{MULTISCALE HEAT AND MASS TRANSFER IN PHASE CHANGE SYSTEMS}

In many engineering systems, the discontinuity of materials is not considered since the scale of the material is large enough to obtain the bulk material properties from the average behavior of its nano/microstructure. The continuous behavior of the material is defined by mass $m$, which is expressed as a continuous function of volume by density. In other words, the nano/microscopic discontinuous distribution of mass is reestablished by density which is a continuous mass distribution in space. Based on the continuum hypothesis, governing equations for large-scale engineering systems are derived from the combination of constitutive law and conservation principles of mass, momentum and energy which work perfectly for the macroscale. These governing equations are usually introduced by a group of partial differential equations and are coupled with boundary or initial conditions to solve either a boundary value system or initial boundary value system. To solve a specific boundary or initial value system showing the continuous behavior, various analytical and numerical methods are available such as finite difference method (FDM), finite volume method (FVM), and finite element method (FEM).

In macroscale engineering systems, the mean free path of molecules is very small compared with the characteristic length of the system, and therefore, many engineering systems are properly defined by a hypothetical continuum model. However, there are some engineering systems which do not appropriately fit into the continuum assumption. For instance, to describe a reservoir is being filled by different particles, the shapes, dimensions, mass, and the interaction between particles and wall should be individually considered. For each particle, the interaction law is coupled with momentum balance to 
provide governing equations describing that particle. Therefore, in a discontinuum system, a set governing equations of all particles are solved to determine the final state of each particle, while in a continuum system, the number of governing equations does not depend on the size of the system [18].

In the discontinuum (nano/microscale) system, the number of equations is determined by the number of particles. Discontinuum systems have a rarely analytical solution. There are several numerical methods such as Monte Carlo, discrete element method, discontinuous deformation analysis, and molecular dynamics method that are able to find an approximation of the solution of the discontinuum systems. In Monte Carlo molecular modeling, statistical mechanics is adopted to find the equilibration condition of the system, without considering the time required for the equilibrium. Discrete element methods and discontinuous deformation analysis use grain-scale particles with friction interactions . In molecular dynamics method, the atom interactions are calculated using the second law of Newton, where the atoms are assumed to exist in a force field determining the forces between different atoms. This method was first developed by Fermi, Pasta, Ulam and Tsingou in the mid-1950s after the effectiveness of Monte Carlo simulation was approved. By advancement of computing performance and parallel programming, LAMMPS ( Large-Scale Atomic/Molecular Massively Parallel Simulator) was developed for the molecular dynamics program in 2001 by Sandia National Laboratories [19].

Currently, multi-scale phase change systems are used in different applications such as water purification, water harvesting, and thermal management. In water purification technology, one of the multi-scale phase change systems is membrane distillation. This membrane is running on the partial pressure gradient due to non-isothermal process 
through a nanoporous membrane. In the membrane distillation, water flows inside mili channels with truly continuum behavior, but water is evaporated at the interface of the channel and membrane and conducted through nanoporous membrane which does not follow continuum behavior. The pore size of the membrane ranges from several nanometers to a few micrometers depending on liquid entry pressure and permeability. The liquid entry pressure, the minimum hydrostatic pressure required for feed solution to enter the pore, should be as high as possible requiring small pore size. However, the pore size of the membrane should be big to deliver high water production. Therefore, appropriate pore size and distribution should be chosen to compromise between high liquid entry pressure and permeability [20].

The membrane channel diameter (pore size) is critical in the transportation of the molecules through the membrane as shown in Fig. 1.4. There are two different mechanisms to describe the transport of the molecules in membrane distillation including Knudsen and Molecular diffusions. If the mean free path of the transported molecules is higher than the membrane channel diameter, the collisions between the molecules and the flow channel wall is dominant compared to the collisions between the molecules, and the mass transport is governed by Knudsen diffusion. If the mean free path of the transported molecules is smaller than the membrane channel diameter, the mass transport is described by molecular diffusion. 


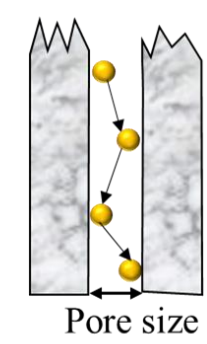

Knudsen diffusion

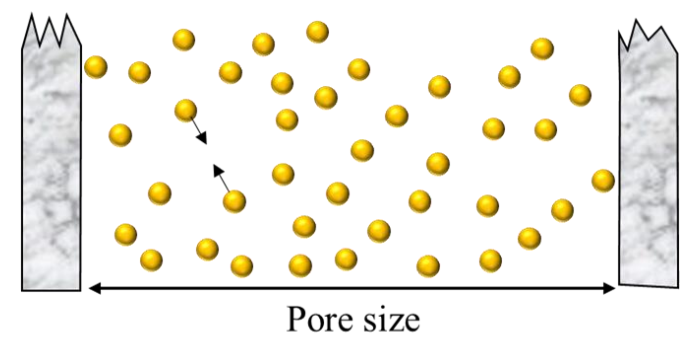

Molecular diffusion

\section{Figure 1.4 Schematic representations of Knudsen and molecular diffusions}

Scaling is one of the important technical issues to address in membrane desalination technologies where the high brine concentrations close to its saturation limit are used. In a membrane distillation using highly concentrated solutions, salt crystals are formed and deposited on the membrane surface in the feed channel due to oversaturation, resulting in the blockage of the opening area of the membrane as illustrated in Fig. 1.5. This blockage causes a rapid reduction in the distilled water production. The scaling is another example of the multiscale systems in which the nuclei of salt crystals are created in a nanoscale size. If the nuclei are stable, they grow to microscale, until the resultant force from the solution and membrane leads to the deposition of the crystals on the membrane surface. The modeling of the salt scaling is challenging and requires taking all forces exerted on salt crystals from the solution and membrane into account. 


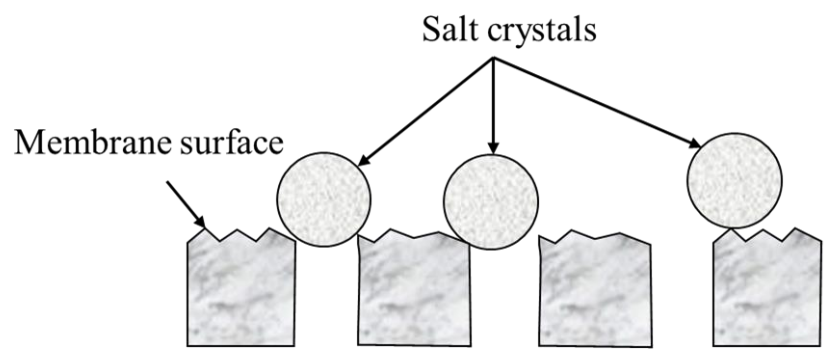

Figure 1.5 Schematic representation of crystal deposition 


\section{CHAPTER 2: DIRECT CONTACT MEMBRANE DISTILLATION USING DIESEL ENGINE WASTE HEAT}

Critical off-grid locations often use diesel power generators as a means of producing portable and reliable electricity. Diesel generators are compact, quickly deployable, and energy-efficient, and generate immense amounts of waste heat. Harvesting the waste heat from the diesel generators can provide a reliable and consistent source of heat. In fact, over 50\% of the energy from diesel fuel is wasted in the form of low and high-grade heat by the engine cooling system and exhaust, respectively. Therefore, because of the combined demand for potable water and electrical power in disaster-stricken areas, it is reasonable to employ the MD system running on the waste heat of diesel generators. Here, a numerical analysis using a thermal-hydraulic network model was performed for the DCMD systems running on the waste heat of the diesel engine cooling system to investigate the effects of various operating and design variables of the DCMD systems on water distillation performance and thermal efficiency.

\subsection{NUMERICAL METHOD}

The schematic of the DCMD system considered for this study is depicted in Fig. 2.1(a). The DCMD system consists of a feed loop and a permeate loop connected by a nanoscale porous, hydrophobic membrane. Saline water is considered as feed water for the DCMD system. Each loop consists of a heat exchanger and an external connection: a heat exchanger (HX1) and brine influx $\left(\dot{m}_{b}\right)$ in the feed loop, and a heat exchanger (HX2) and distillate output $\left(\dot{m}_{d}\right)$ in the permeate loop. The brine influx and distillate output are at an equal flow rate for the mass balance of the DCMD system. The heat exchanger (HX1) 
receives the heat $\left(Q_{i n}\right)$ from the diesel engine coolant (heat source) for the feed loop, while the heat exchanger (HX2) dissipates the same amount of heat $\left(Q_{\text {out }}\right)$ to ambient (heat sink) for the permeate loop. Therefore, it satisfies the energy balance of the DCMD system.

For the DCMD membrane in Fig. 2.1(a), phase change occurs in the hydrophobic pores of the membrane surfaces in direct contact with the feed and permeate streams at its respective vapor pressure: evaporation $\left(q_{f g}\right)$ on the feed side of the membrane and condensation $\left(q_{f g}\right)$ on the permeate side. The difference between the vapor pressures set by the respective concentration of the feed stream and saturation temperatures of the feed and permeate streams is the driving force of the water vapor flow through the membrane. A parasitic conduction heat transfer, $q_{k}$ (heat loss) across the solid phase of the membrane is unavoidable with any temperature difference between the fluid streams. The sensible heat carried by the feed and permeate streams flowing into the membrane is used for the latent heat required for the phase change for water distillation as well as the conduction loss. Figure 2.1(b) shows the schematic of a DCMD system consisting of $N_{m}$ membrane modules of the same length connected in parallel which was used in analysis with the results presented in Fig. 2.11. The main flows of the feed and permeate streams are equally divided into each membrane module flowing in a counterflow configuration.

The following assumptions were used in the numerical analysis of the DCMD system using a thermal-hydraulic network model.

(i) There are no polarization effects for velocities, temperatures and concentrations of feed and permeate streams in the membrane channels which are filled with a mesh spacer. That is, the lateral variations of velocity, temperature and concentration are negligible and vary along only $x$-direction (flow directions). Therefore, the local fluid 
velocity, temperature and concentration on the membrane equals the bulk fluid velocity, temperature and concentration in the membrane channels, respectively. But the water vapor flow through the membrane perpendicular to the fluid flow direction in the membrane channels is counted in this one-dimensional analysis.

The one-dimensional approach is justifiable by the fact that a mesh spacer installed in the feed and permeate channels to physically support the fragile membrane, creates strong "turbulent mixing" as the fluid flows through tortuous paths in the mesh-filled channels and therefore greatly enhances the heat and mass transfer in the channels. Phattaranawik [21] reported an experimental measurement result of the temperature polarization coefficient of spacer-filled channels to be in the range of 0.9-0.97 which means the channel flow are well mixed in a lateral direction (along the channel height), and thus, the temperature gradient in the channels are negligible in the lateral direction. Furthermore, several researchers [22, 23] reported the concentration polarization coefficient (the ratio of the wall concentration to the bulk concentration) is in the range of 1.04-1.1 which suggests a negligible concentration polarization effect. Such an analogy between momentum, heat and mass transfer are well established by a boundary layer theory [24].

(ii) Liquid flow-through across the membrane does not occur due to a high capillary pressure head in the nanopores in the hydrophobic membrane made of PTFE.

(iii)A complete rejection of salt takes place in the DCMD and thus no trace of salt is found in the permeate water. 


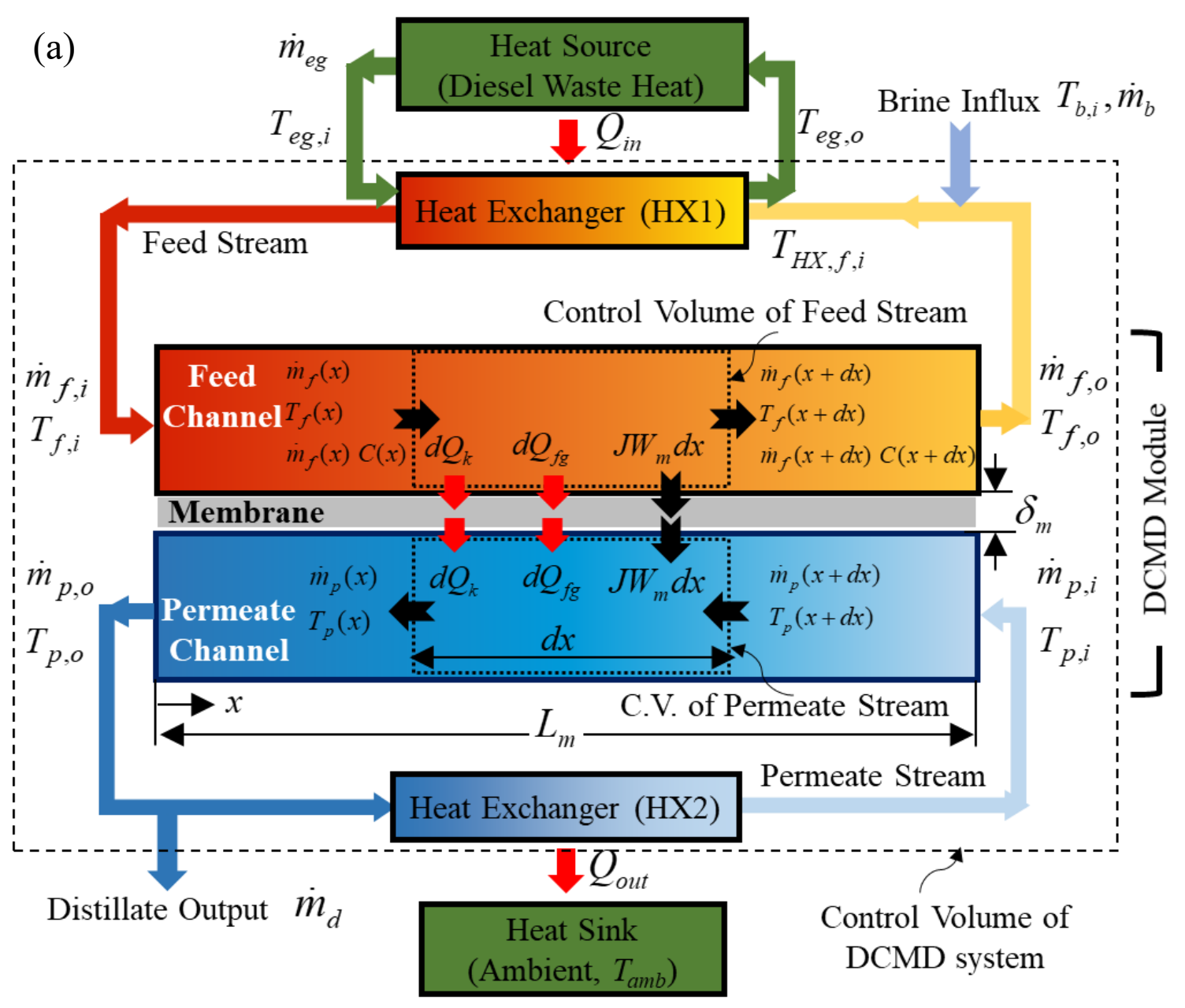

(b)

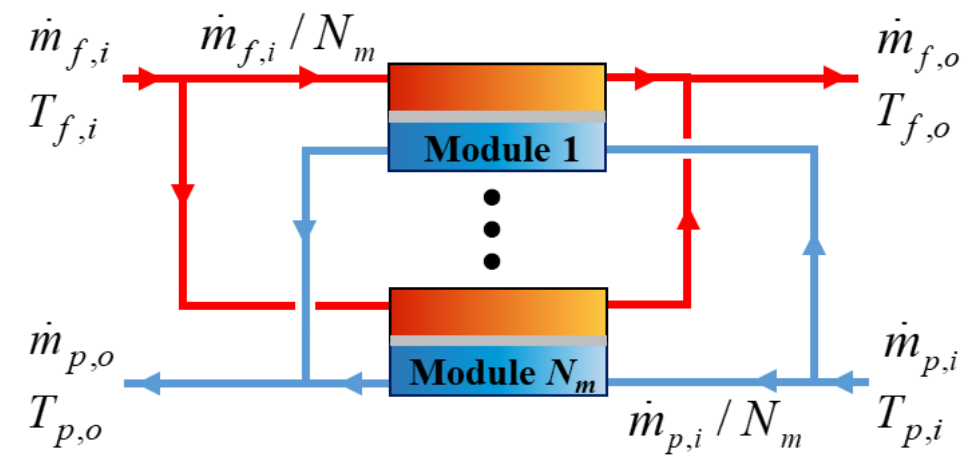

Figure 2.1 (a). Schematic of a single-module DCMD system used for thermalhydraulic network modeling. (b) Schematic of a multiple-module DCMD system connected in a parallel arrangement 
A one-dimensional formulation was used for the numerical analysis based on the aforementioned assumptions. The governing equations for mass, energy and concentration conservations can be expressed as a function of only the $x$ variable which is aligned with the feed flow direction but opposite the permeate flow. The mass conservation equations of the feed and permeate streams in an infinitesimal control volume [dotted boxes in Fig. 2.1(a)] are given by

$$
\begin{aligned}
& \frac{\partial \dot{m}_{f}}{\partial x}=-J W_{m}, \\
& \frac{\partial \dot{m}_{p}}{\partial x}=-J W_{m},
\end{aligned}
$$

where $W_{m}$ is the widths of the membrane channels set to $1 \mathrm{~m}$ for simplicity. The height of the channels is not required to model the one-dimensional flow network.

The energy conservation equations for the feed and permeate streams in the membrane channels are given by

$$
\begin{aligned}
& \dot{m}_{f} c_{w} \frac{\partial T_{f}}{\partial x}=c_{w} J W_{m} T_{f}-J W_{m} h_{f g}-k_{m} W_{m} \frac{T_{f}-T_{p}}{\delta_{m}} \\
& \dot{m}_{p} c_{w} \frac{\partial T_{p}}{\partial x}=c_{w} J W_{m} T_{p}-J W_{m} h_{f g}-k_{m} W_{m} \frac{T_{f}-T_{p}}{\delta_{m}}
\end{aligned}
$$

where $J$ is the water mass flux, $h_{f g}$ is the latent heat of evaporation or condensation, and $\delta_{m}$ is the thickness of the membrane. $k_{m}$ is the effective thermal conductivity of the membrane and calculated by

$$
k_{m, e}=\left(1-\varepsilon_{m}\right) k_{m}+\varepsilon_{m} k_{g},
$$


where $\varepsilon_{m}, k_{m}$, and $k_{g}$ are the porosity and bulk thermal conductivity of the membrane material, and thermal conductivity of water vapor, respectively.

The salt concentration in the feed channel is assumed to vary along only the flow $(x)$ direction because of a negligible concentration polarization effect due to a strong flow mixing by a mesh spacer and can be determined based on a one-dimensional salt conservation equation and is given by

$$
\frac{\partial C}{\partial x}=\frac{J W_{m}}{\dot{m}_{f}} C
$$

where $C$ is the salt concentration in the feed channel of the MD system. To determine $J$ in Eq . (2.6), the pore size of the membrane and mean free path of the water vapor or air are critical. The main variable determining the actual mass transport mechanism in a certain membrane pore is Knudsen number $(\mathrm{Kn})$ expressed as the ratio of the mean free path of the transported molecules to the pore size of the membrane.

$$
\mathrm{Kn}=\frac{\lambda}{d_{p}}
$$

For a binary mixture of water vapor and air. The mean free path of water vapor in the air $\left(\lambda_{w v-a}\right)$ is determined at the average membrane temperature

$$
\lambda_{w v-a}=\frac{k_{B} T_{m}}{\pi\left[\left(\sigma_{w v}+\sigma_{a}\right) / 2\right]^{2} p_{T}} \frac{1}{\sqrt{1+\left(M_{w} / M_{a}\right)}},
$$

where $k_{B}$ is the Boltzmann constant, $p_{T}$ the total pressure $\left(1.013 \times 10^{5} \mathrm{~Pa}\right.$ for DCMD), and $\sigma_{w v}$ and $\sigma_{a}$ is the collision diameter of the water vapor $\left(2.641 \times 10^{-10} \mathrm{~m}\right)$ and air $\left(3.711 \times 10^{-}\right.$ $\left.{ }^{10} \mathrm{~m}\right)$, and $M_{w}$ and $M_{a}$ the molecular weights of water and air. 


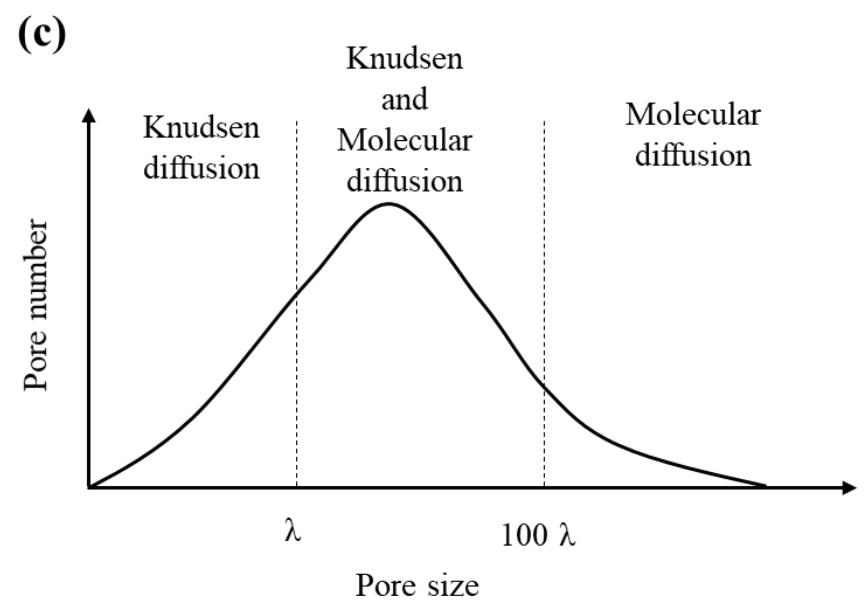

Figure 2.2 Mechanism of mass transport of the membrane distillation with pore size distribution

If Knudsen is greater than one $\left(\mathrm{Kn}>1\right.$, or $\left.d_{p}<\lambda\right)$. It means that the mean free path of the transported molecules is large in comparison with the membrane pore size, the collisions of the molecule-pore overcomes the collisions of molecule-molecule, thus, the molecules are transported by Knudsen transport. If Knudsen is smaller than 0.01 $(\mathrm{Kn}<0.01)$, the mass transport through the membrane pore is manipulated by molecular diffusion of trapped air inside the membrane pore because of low solubility of air in the water. In the transition region, $0.01,0.01<\mathrm{Kn}<1$, both molecular diffusion and Knudsen diffusion have a contribution to the mass transport inside the pore. The combination of molecular and Knudsen diffusion is used to define the permeability of each membrane pore. Phattaranawik et. al. [25] considered the effect of pore size distribution for the commercial membranes as shown in Fig. 2.2. They used lognormal distribution for the pore sizes as 


$$
n\left(d_{p}\right)=\frac{1}{S D_{\log } d_{p} \sqrt{2 \pi}} \exp \left[-\left(\frac{\ln \left(d_{p} / \bar{d}_{m}\right)}{\sqrt{2} S D_{\log }}\right)^{2}\right],
$$

where $n\left(d_{p}\right)$ is the number of pores with diameter of $d_{p}, d_{m}$ the mean pore diameter, and $S D_{\text {log }}$ the standard deviation of lognormal function. Regarding the practical mean free path in the MD, the area of lognormal distribution for pure ordinary diffusion is less than $1 \%$. Thus, the effect of purely molecular diffusion can be ignored. To obtain the total fluxes, the Knudsen and transition contribution are integrated in each region over the pore size distribution.

$$
\bar{J}_{T}=\frac{\int_{d_{p}=0}^{d_{p}=\lambda} J_{\mathrm{Kn}} \frac{\pi d_{p}^{2}}{4} n\left(d_{p}\right) \mathrm{d} d_{p}+\int_{d_{p}=\lambda}^{d_{p}=\infty} J_{\mathrm{Tr}} \frac{\pi d_{p}^{2}}{4} n\left(d_{p}\right) \mathrm{d} d_{p}}{\int_{d_{p}=0}^{d_{p}=\infty} \frac{\pi d_{p}^{2}}{4} n\left(d_{p}\right) \mathrm{d} d_{p}},
$$

The average pore sizes are calculated and the molar flux for each region are obtained at the location of $50 \%$ of the number of pores in each region. By substituting the average pore size and its molar flux, the total molar flux can be rewritten as

$$
\bar{J}_{T}=\frac{\bar{J}_{\mathrm{Kn}} \int_{d_{p}=0}^{d_{p}=\lambda} \frac{\pi d_{p}^{2}}{4} n\left(d_{p}\right) \mathrm{d} d_{p}+\bar{J}_{\operatorname{Tr}} \int_{d_{p}=\lambda}^{d_{p}=\infty} \frac{\pi d_{p}^{2}}{4} n\left(d_{p}\right) \mathrm{d} d_{p}}{\int_{d_{p}=0}^{d_{p}=\infty} \frac{\pi d_{p}^{2}}{4} n\left(d_{p}\right) \mathrm{d} d_{p}} .
$$

Equation (2.11) is not simple enough. For more simplification, the fractions of the membrane area are assumed to be the fraction of the number of pores in each region 
multiplied by the square of the mean pore diameter. Therefore, the fractions of the pore number are obtained by the cumulative distribution function of lognormal distribution $(\Phi)$.

$$
\begin{aligned}
& \int_{d_{p}=0}^{\frac{d_{p}=0}{d_{p}=\infty} \frac{\pi d_{p}^{2}}{4} n\left(d_{p}\right) \mathrm{d} d_{p}} \approx\left(\frac{\bar{d}_{\mathrm{Kn}}}{\bar{d}_{m}}\right)^{2} \int_{d_{p}=0}^{\left.\int_{d_{p}=0}^{d_{p}=\lambda} n\left(d_{p}\right) \mathrm{d} d_{p}\right) \mathrm{d} d_{p}}=\left(\frac{\bar{d}_{\mathrm{Kn}}}{\bar{d}_{p}}\right)^{2} \frac{n_{\mathrm{Kn}}}{n_{\mathrm{T}}}=\left(\frac{\bar{d}_{\mathrm{Kn}}}{\bar{d}_{p}}\right)^{2} \Phi\left(\frac{\ln \left(\lambda / \bar{d}_{p}\right)}{\mathrm{SD}_{\log }}\right),
\end{aligned}
$$

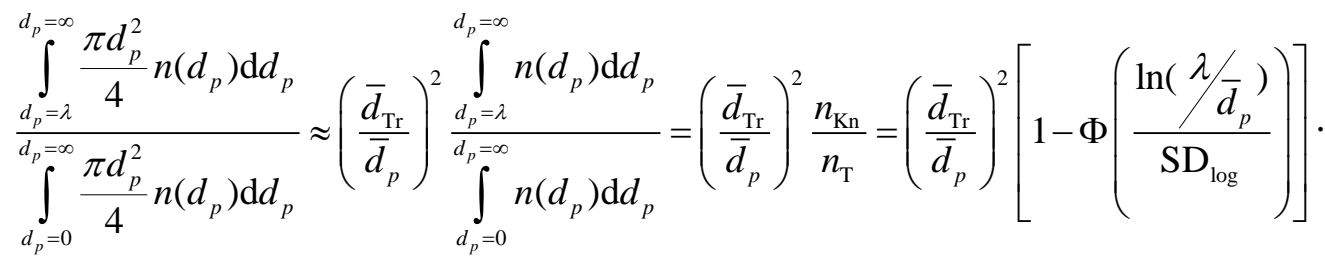

By substituting Eq. (2.12) and (2.13) into Eq. (2.11), the correlation of mass transfer obtained from the idea of the pore size distribution

$$
\bar{J}_{T}=\left(\frac{\bar{d}_{\mathrm{Kn}}}{\bar{d}_{\mathrm{Tr}}}\right)^{2} \Phi\left(\frac{\ln \left(\lambda / \bar{d}_{p}\right)}{\mathrm{SD}_{\log }}\right) \bar{J}_{\mathrm{Kn}}+\left(\frac{\bar{d}_{T r}}{\bar{d}_{m}}\right)^{2}\left[1-\Phi\left(\frac{\ln \left(\lambda / \bar{d}_{p}\right)}{\mathrm{SD}_{\log }}\right)\right] \bar{J}_{T r} .
$$

The cumulative distribution function in Eq. (2.14) is the weight fractions representing the significance of each region. It is noteworthy that other distribution functions can also be used. Fick's law is applied to derive the molar mass of membrane with the existence of the air as follows

$$
J_{m o l}=\frac{\varepsilon D_{w v-a}}{\tau R T} \nabla p+\frac{p_{w v}}{p_{a}}\left(J_{m o l}+J_{a}\right) .
$$

The effect of the air flux is in the form of counter-molecular diffusion and the relationship between $J_{m o l}$ and $J_{a}$ can be obtained by Graham's law 


$$
\frac{J_{a}}{J_{m o l}}=-\sqrt{\frac{M_{w}}{M_{a}}} .
$$

The correlation of molecular diffusion by considering air flux is determined by combination of Eqs. (2.13) and (2.14).

$$
J_{m o l}=\frac{\left(\varepsilon_{m} / \tau_{m}\right) D_{w v-a}}{R T_{m}\left[1-\frac{p_{w v}}{p_{T}}(1-\alpha)\right]} \nabla p
$$

The series combination of Knudsen and molecular diffusion are given by

$$
\frac{\nabla p}{\bar{J}_{T r}}=\frac{\nabla p}{J_{m o l}}+\frac{\nabla p}{J_{\mathrm{Kn}}} .
$$

The Knudsen diffusion can be written by:

$$
J_{\mathrm{Kn}}=\frac{4}{3} \frac{\varepsilon_{m} d_{p}}{R T_{m} \tau_{m}} \sqrt{\frac{R T_{m}}{2 \pi M_{w}}} \nabla p,
$$

where $\tau_{m}$ is pore tortuosity. The diffusive flux of water vapor in membrane distillation is determined by combination of Eqs. (2.17), (1.18) and (1.19) as follows

$$
J_{T r}=-\frac{1}{R T}\left(\frac{p_{T}-(1-\alpha) p_{w v}}{\left(\varepsilon_{m} / \tau_{m}\right) p_{T} D_{w v-a}}+\frac{3}{4} \frac{\tau_{m}}{\varepsilon \bar{d}_{p}}\right)^{-1} \nabla p
$$

By integrating Eq. (2.20), the steady state flux of water vapor in transition condition is calculated by

$$
J=\frac{\left(\varepsilon_{m} / \tau_{m}\right) p_{T} D_{w v-a} M_{w}}{(1-\alpha) \delta_{m} R T_{m}} \ln \left\{\frac{D_{K n}\left[p_{T}-(1-\alpha) p_{v, p}\right]+\left(\varepsilon_{m} / \tau_{m}\right) p_{T} D_{w v-a}}{D_{K n}\left[p_{T}-(1-\alpha) p_{v, f}\right]+\left(\varepsilon_{m} / \tau_{m}\right) p_{T} D_{w v-a}}\right\},
$$




$$
\begin{aligned}
& D_{K n}=\frac{4 \varepsilon_{m}}{3 \tau_{m}} d_{p} \sqrt{\frac{R T_{m}}{2 \pi M_{w}}}, \\
& p_{T} D_{w v-a}=1.895 \times 10^{-5} T_{m}^{2.072}, \\
& \alpha=\frac{M_{w}}{M_{a}}
\end{aligned}
$$

where $\varepsilon_{m}, \tau_{m}, \delta_{m}$ and $d_{p}$ are porosity, tortuosity, thickness, and pore diameter of the membrane, respectively and their values are listed in Table 2.1. $D_{K n}$ is the diffusivity for Knudsen diffusion. $p_{T} D_{w v-a}$ is a product of the total pressure $\left(p_{T}\right)$ and water-vapor diffusivity $\left(D_{w v-a}\right)$ in the air and has the unit of $\left[\mathrm{Pa} \mathrm{m}^{2} \mathrm{~s}^{-1}\right] . T_{m}$ is the membrane temperature in Kevin and determined by the average of the local feed and permeate temperatures $\left(T_{m, f}\right.$, $\left.T_{m, p}\right)$ on the membrane surfaces. $M_{w}$ and $M_{a}$ are the molecular weights of water and air, respectively.

Table 2.1 Dimensional and thermophysical properties of membrane and baseline operating conditions and dimension of Direct Contact Membrane Distillation system

Dimensional and thermophysical properties of membrane

\begin{tabular}{cccccc}
\hline $\begin{array}{c}\text { Membrane } \\
\text { type }\end{array}$ & $\delta_{m}(\mathrm{~m})$ & $d_{p}(\mathrm{~m})$ & $\varepsilon_{m}$ & $\tau_{m}$ & $k_{m}\left(\mathrm{Wm}^{-1} \mathrm{~K}^{-1}\right)$ \\
\hline QM022 & $67 \times 10^{-6}$ & $3.6 \times 10^{-7}$ & 0.8 & 1.79 & 0.23 \\
\hline \multicolumn{6}{l}{ Baseline operating conditions and dimensions of DCMD module } \\
\hline$T_{f, i}\left({ }^{\circ} \mathrm{C}\right)$ & $T_{p, i}\left({ }^{\circ} \mathrm{C}\right)$ & $\begin{array}{c}\dot{m}_{f}=\dot{m}_{p} \\
\left(\mathrm{~kg} \mathrm{~s}^{-1}\right)\end{array}$ & $\begin{array}{c}L_{m} \\
(\mathrm{~m})\end{array}$ & $\begin{array}{c}W_{m} \\
(\mathrm{~m})\end{array}$ & $C_{f, i}\left(\mathrm{gr} \mathrm{L}^{-1}\right)$ \\
\hline 70 & 40 & 1 & 1 & 1 & 35 \\
\hline
\end{tabular}


In Eq. (2.21), the vapor pressures $\left(p_{v, f}, p_{v, p}\right)$ of the feed and permeate streams are evaluated at their local fluid temperatures. For non-ideal binary mixtures with the nonvolatile solute $(\mathrm{NaCl})$, the partial pressure can be determined by

$$
p_{v}=a_{w}(1-M) p_{s a t}
$$

where the water activity in $\mathrm{NaCl}$ solutions, $a_{w}$, is a function of the composition and determined by [26]

$$
a_{w}=1-0.5 M-10 M^{2}
$$

where $M$ is the mole fraction of $\mathrm{NaCl}$ solutions. The pure water saturation pressure $\left(p_{\text {sat }}\right)$ in the unit of Pascal can be determined by the Antoine equation and is given by [27]

$$
p_{\text {sat }}=\exp \left(23.1964-\frac{3816.44}{T-46.13}\right)
$$

where $T$ is the fluid temperature in the unit of Kelvin. The presence of salt in the feed water decreases the water vapor pressure in proportion to the salt concentration, as shown in Eq. (2.11). For seawater with a mole fraction of $0.01(C=35 \mathrm{gr} / \mathrm{L})$, the vapor pressure is decreased by $1.6 \%$ at $60^{\circ} \mathrm{C}$.

The thermal efficiency of the DCMD system can be defined by the ratio of the phase change heat transfer to the total heat transfer across the membrane which is used to measure the efficiency of the thermal energy utilization of the membrane distillation and is given by

$$
\eta_{t}=\frac{q_{f g}}{q_{f g}+q_{k}}
$$

where, 


$$
\begin{aligned}
& q_{f g}=W_{m} \int_{0}^{L_{m}} q_{f g}^{\prime \prime} d x=W_{m} \int_{0}^{L_{m}} J h_{f g} d x \\
& q_{k}=W_{m} \int_{0}^{L_{m}} q_{k}^{\prime \prime} d x=W_{m} \int_{0}^{L_{m}} \frac{k_{m}}{\delta_{m}}\left(T_{f}-T_{p}\right) d x
\end{aligned}
$$

The diesel engine loses a large portion of the combustion heat through engine cooling (about $20 \%$ of the fuel energy) and exhaust gas (about $30 \%$ of the fuel energy). In this analysis, the engine coolant is considered as the heat source for the DCMD system. A flat plate heat exchanger (HX1) is used to exchange the heat between the engine coolant and the feed stream in a counter-flow arrangement. The dimensions of the flat plate heat exchanger are listed in Table 2.2. The technical specifications [28] of a commercial diesel generator (Cummins, model KTA38-G9) reports that $672 \mathrm{~kW}$ is ejected to the engine cooling system and the temperature and flow rate of the engine coolant available for the heat exchanger are $110^{\circ} \mathrm{C}$ and $6.8 \mathrm{~kg} / \mathrm{s}$, respectively.

Table 2.2 Dimensions and thermophysical property of the flat-plate heat exchanger (HX1) used for diesel engine cooling system

\begin{tabular}{ccccc}
\hline$L_{H X}(\mathrm{~m})$ & $W_{H X}(\mathrm{~m})$ & $a_{p}(\mathrm{~m})$ & $\delta_{p}(\mathrm{~m})$ & $k_{p}\left(\mathrm{Wm}^{-1} \mathrm{~K}^{-1}\right)$ \\
\hline 0.87 & 0.38 & $3 \times 10^{-3}$ & $0.42 \times 10^{-3}$ & 13.2 \\
\hline
\end{tabular}

The energy supply $\left(q_{\text {in }}\right)$ from the heat source heat exchanger (HX1) required for the DCMD system can be determined from an energy balance and is given by

$$
q_{i n}+\dot{m}_{b, i} c_{w} T_{b, i}=\dot{m}_{f, i} c_{w} T_{f, i}-\left(\dot{m}_{f, i}-\dot{m}_{b, i}\right) c_{w} T_{f, o}=N_{m}\left(q_{f g}+q_{k}\right)
$$

where $N_{m}$ is the number of membrane modules in the DCMD system. For the one-module system, $N_{m}=1$. 
From an energy balance, the temperature of the mixed flow of the brine inflow and feed water entering into the heat source heat exchanger (HX1) is determined by

$$
\dot{m}_{f, o} c_{w} T_{f, o}+\dot{m}_{b} c_{w} T_{b, i}=\dot{m}_{f, i} c_{w} T_{H X, f, i}
$$

The energy balance for the heat exchanger (HX1) is described by

$$
q_{i n}=\dot{m}_{f, o} c_{w}\left(T_{f, i}-T_{H X, f, i}\right)=\dot{m}_{e g} c_{e g}\left(T_{e g, i}-T_{e g, o}\right)
$$

The heat transfer area of the heat source heat exchanger (HX1) is determined using $\varepsilon$-NTU method [24]. The heat exchanger effectiveness $\left(\varepsilon_{H X}\right)$ is calculated by

$$
\varepsilon_{H X}=\frac{C_{h}\left(T_{h, i}-T_{h, o}\right)}{C_{\min }\left(T_{h, i}-T_{c, i}\right)}
$$

where the subscript $h$ is for the hot fluid (feed stream) and the subscript $c$ is for cold fluid (permeate stream). For a heat exchanger in a counterflow configuration, the relation for $\varepsilon$ and NTU is given by

$$
\varepsilon_{H X}=\frac{1-\exp \left[-\mathrm{NTU}\left(1-C_{r}\right)\right]}{1-C_{r} \exp \left[-\mathrm{NTU}\left(1-C_{r}\right)\right]}
$$

where NTU is the number of the unit. $C_{r}$ is the ratio between the minimum and maximum heat capacity rates. $U$ is the overall heat transfer coefficient. NTU and $U$ are determined by

$$
\begin{aligned}
& \mathrm{NTU}=\frac{U A}{C_{\min }} \\
& \frac{1}{U}=\frac{1}{h_{h}}+\frac{\delta_{p}}{k_{p}}+\frac{1}{h_{c}}
\end{aligned}
$$


where $C_{\min }$ is the minimum heat capacity. $h_{h}$ and $h_{c}$ are the hot-side and cold-side convective heat transfer coefficients, respectively. $\delta_{p}$ and $k_{p}$ are the thickness and thermal conductivity of the plate walls of the heat exchanger, respectively, and their values are listed in Table 2.2.

The heat source heat exchanger (HX1) was assumed to be a flat plate heat exchanger [29]. The heat transfer correlations of the flat plate heat exchanger used to calculate the convective heat transfer coefficients $\left(h_{h}, h_{c}\right)$ are given by

$$
\begin{aligned}
& \mathrm{Nu}=\frac{h D_{h}}{k_{f}}= \begin{cases}1.67 \operatorname{Re}^{0.44} \operatorname{Pr}^{0.5} & 45<\operatorname{Re}<300 \\
0.405 \operatorname{Re}^{0.7} \operatorname{Pr}^{0.5} & 300<\operatorname{Re}<2000 \\
0.84 \operatorname{Re}^{0.6} \operatorname{Pr}^{0.5} & 2000<\operatorname{Re}<20000\end{cases} \\
& \operatorname{Re}=\frac{\left(\frac{2 \dot{m}}{N_{H X}}\right) D_{h}}{\mu A_{c}}
\end{aligned}
$$

where $\dot{m}$ is the flow rate of the feed stream or engine coolant. $N_{H X}$ is the number of the flow channels, $A_{c}$ is the cross-sectional area of the flow channels, and $D_{h}$ is the hydraulic diameter of the flow channel in the flat plate heat exchanger. $\mu$ is the dynamic viscosity and $k_{f}$ is the thermal conductivity and Pr is Prandtl number of the fluids. Using the dimensions of the flat plate heat exchanger in Table 2.2, $A_{c}$ and $D_{h}$ are determined by

$$
\begin{aligned}
& A_{c}=a_{p} W_{H X} \\
& D_{h}=\frac{4 a_{p} W_{H X}}{2\left(a_{p}+W_{H X}\right)}
\end{aligned}
$$


where $a_{p}$ and $W_{H X}$ are the channel height of the heat exchanger (the gap between the plates) and the width of the plate for the flat plate heat exchanger.

\subsection{RESULTS AND DISCUSSIONS}

A Direct Contact Membrane Distillation (DCMD) system running on diesel waste heat was numerically analyzed using a thermal-hydraulic network model. The DCMD system receives the waste heat from a diesel engine cooling system (heat source) via a heat source heat exchanger (HX1) to the feed loop as shown in Fig. 2.1(a). A second heat exchanger (HX2) between the permeate stream and ambient (heat sink) was used to dissipate the heat from the permeate loop to ambient. In this study, the HX2 was assumed to be efficient and capable of maintaining the inlet temperature of the permeate stream $\left(T_{p, i}\right)$ at a set temperature of $40^{\circ} \mathrm{C}$. A counterflow configuration between the feed stream and engine coolant flow in HX1 was used to achieve an efficient heat exchange. The thermophysical properties of the membrane are listed in Table 2.1.

In the first part of this section, a DCMD system consisting of one membrane module system was analyzed to investigate the effects of the inlet temperatures and flow rates of the feed and permeate streams and the membrane length on membrane distillation performance. The results are presented in Fig. 2.2 to 10. The operating conditions used for the analysis are the baseline conditions listed in Table 2.1 save for the variables subject to change. In the second part, another DCMD system consisting of multiple $\left(N_{m}\right)$ membrane modules connected in a parallel arrangement [Fig. 2.1(b)] was analyzed under a constraint that the multiple module DCMD system received a fixed amount of the engine waste heat transferred via HX1 $\left(q_{i n}=672 \mathrm{~kW}\right)$. The results are presented in Fig. 2.10. 


\subsubsection{Effect of Membrane Length}

Figures 2.3 through 2.5 show the effects of the membrane module length $\left(L_{m}\right)$ on the feed and permeate temperature, mass flux, water production rate, and thermal efficiency of the DCMD system. As shown in Fig. 2.3, as the membrane module length (the convective heat transfer area) increased, the outlet temperature of the feed stream decreased, but the outlet temperature of the permeate stream increased. Note that the $x$ direction is aligned with the feed flow direction but opposite the permeate flow direction (i.e., counterflow configuration). Figure 2.3 also shows the variations in the vapor pressure difference $(\Delta p)$ between the feed and permeate streams which rapidly decreased because of the steep change in the saturation pressure of water, especially in the feed stream at higher temperatures, according to the Antoine correlation in Eq. (2.27). In turn, the vapor pressure difference was much higher for shorter modules where a larger temperature difference was maintained between the feed and permeate streams. 


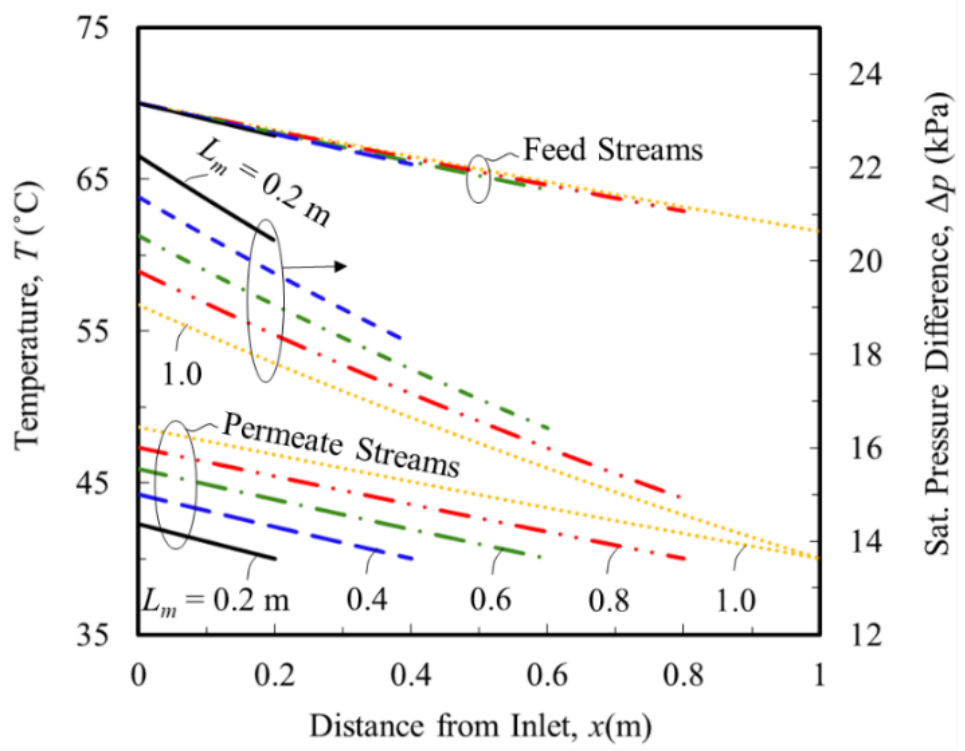

Figure 2.3 Variations of feed and permeate fluid temperatures in a single-module DCMD system and the vapor pressure difference across the membrane with different membrane length under the baseline conditions

Figure 2.4(a) shows that the water mass flux $(J)$ through the membrane was higher for shorter membrane modules and decreased according to the behavior of the vapor pressure difference in Fig. 2.3. The variation of the conduction and phase change heat fluxes along the membrane is shown in Fig. 2.4(b). The conduction heat flux [ $\left.q_{k}^{\prime \prime}=k_{m}\left(T_{m, f}-T_{m, p}\right) / \delta_{m}\right]$ remained constant due to a relatively constant temperature difference between the feed and permeate streams, while the phase change heat flux ( $q_{f g}^{\prime \prime}=J h_{f g}$ ) rapidly decreased as the mass flux does. The heat transfer rates for the conduction and phase change, and thermal efficiency are shown in Fig. 2.5(a). As discussed above, a longer membrane module consumed less heat in the phase change heat transfer, $q_{f g}$, while the conduction heat transfer, $q_{k}$ linearly increased with the membrane length and in turn, the thermal efficiency decreased. Figure 2.5(b) shows that longer membrane modules (larger membrane area) produced more distilled water but at lower thermal 
efficiencies. This result is due to the vapor pressure difference (water mass production) that is reduced, which consumes less heat during the phase change (evaporation and condensation), while the conduction heat loss, which is proportional to the temperature difference across the membrane, remains similar. Such an inefficient use of the heat for the membrane distillation leads to lower thermal efficiency.

(a)

(b)
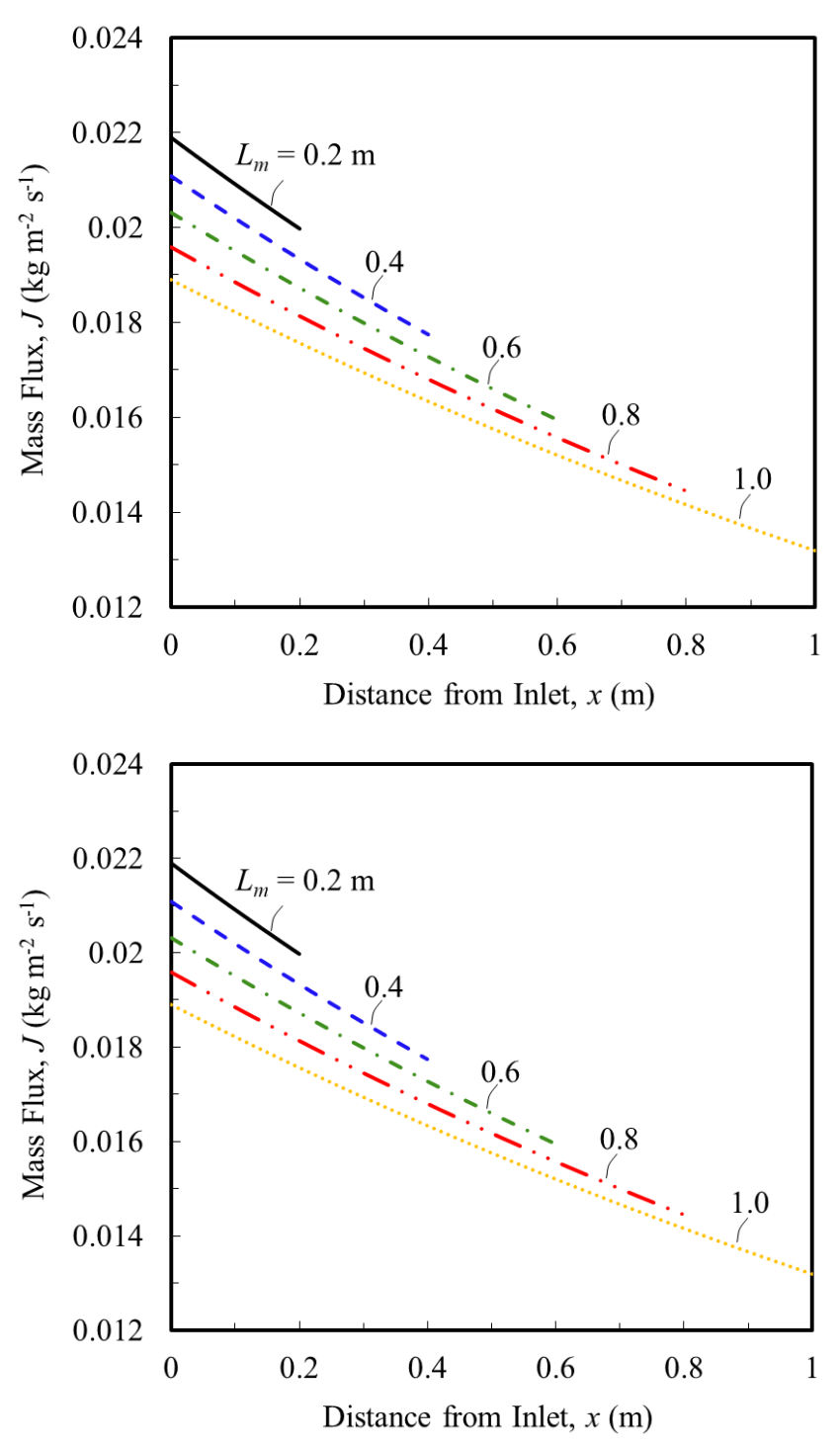

Figure 2.4 (a) Variation of mass flux of water distillate production and (b) conduction and phase change heat fluxes in a single-module DCMD system with different membrane length under the baseline conditions 
(a)

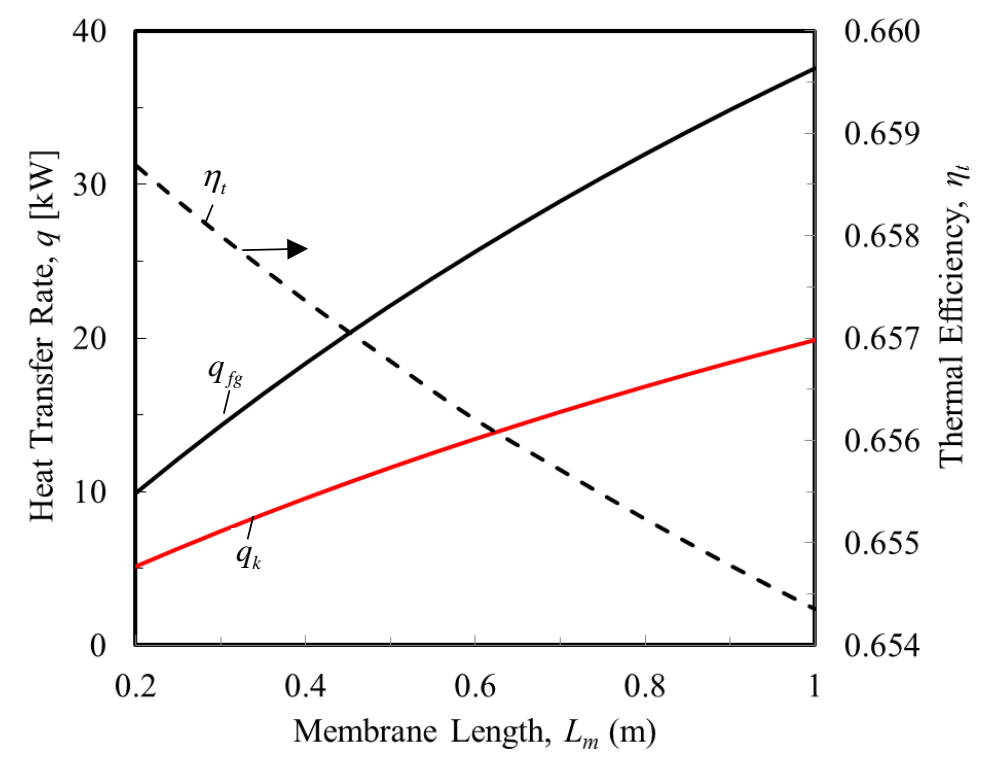

(b)

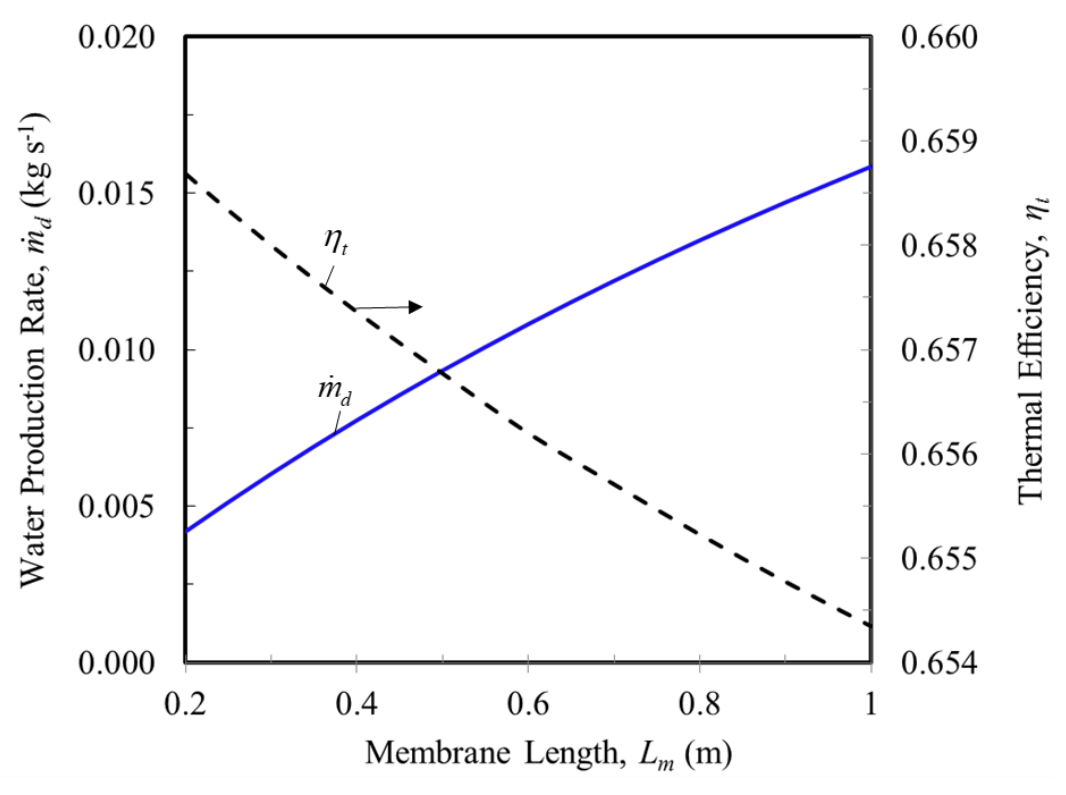

Figure 2.5 Effects of membrane module length on (a) heat transfer rates and thermal efficiency and (b) total water distillate production rate in a single-module DCMD system 


\subsubsection{Effect of Salt Concentration}

Figure 2.6(a) shows the effect of the $\mathrm{NaCl}$ concentration at the inlet of the feed channel on the water mass flux and the concentration variation in the feed channel for the baseline salt concentration. The baseline salt concentration is $35 \mathrm{gr} \mathrm{L}^{-1}$ (mole fraction, $M=$ 0.01) which was chosen based on the salt concentration of seawater. The $\mathrm{NaCl}$ concentration was varied from 0 to $210 \mathrm{gr} \mathrm{L}^{-1}$. The water vapor pressure of the salt solution decreases with the salt concentration according to Eq. (2.25). Therefore, the mass flux decreases with the salt concentration due to the decrease in the vapor pressure of the feed water. Since the reduction in the vapor pressure is compounded as the water is distilled along the feed stream and in turn, the solution concentration gets stronger. Figure 2.6(b) shows that both the water production and thermal efficiency decrease similarly with respect to the salt concentration. 
(a)

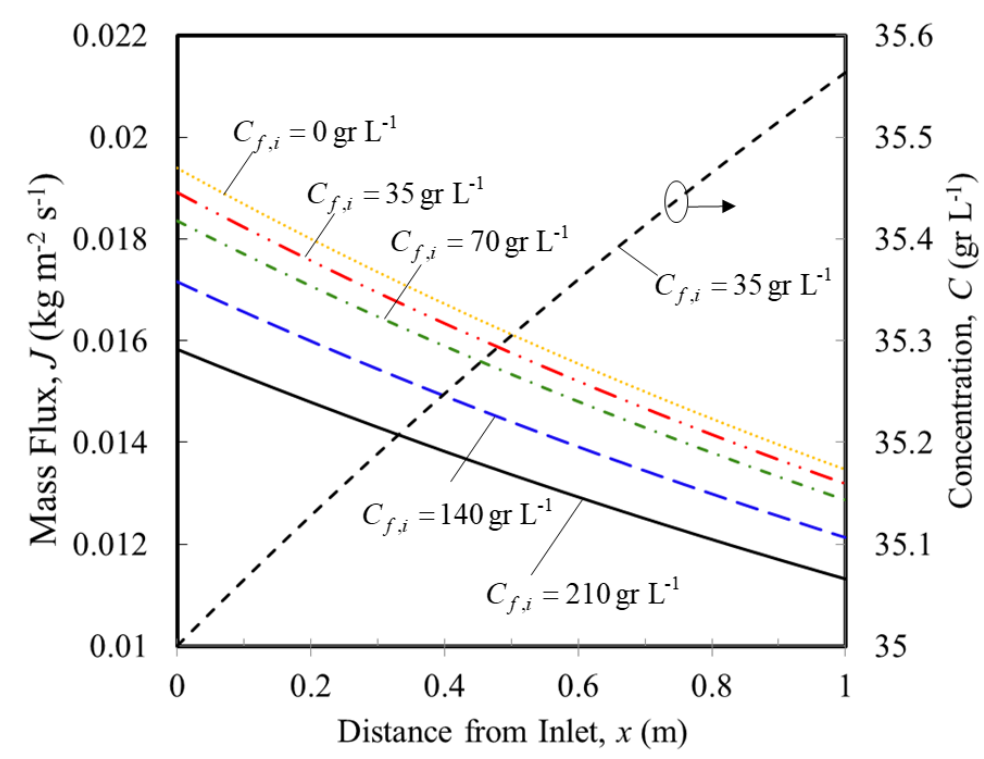

(b)

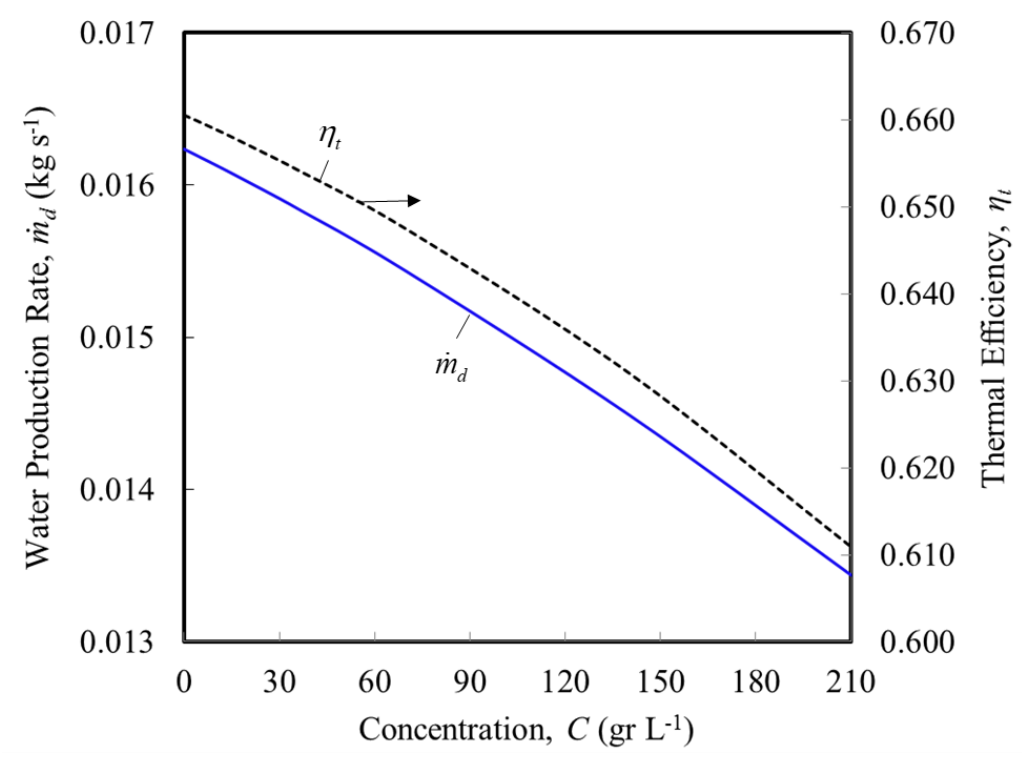

Figure 2.6 (a) Variation of mass flux of water production with different inlet feed concentration and the profile of $\mathrm{NaCl}$ concentration along the $\mathrm{MD}$ channel under the baseline conditions. (b) Effect of inlet feed concentration on thermal efficiency and total water production rate in a single-module DCMD system 


\subsubsection{Effect of Feed and Permeate Water Flow Rate}

The effects of the feed and permeate flow rates were investigated and the results are shown in Figs. 2.7 (a-b) and 2.8 (a-b). For simplicity in the analysis, the feed and permeate flow rates were assumed to be equal. Figure 2.7 shows that as the feed and permeate flow rates (sensible heat) are increased, the temperature variations of the feed and permeate waters will eventually become nearly linear by matching the energy supply and demand for the water production by evaporation and condensation. In Fig. 2.7(b), the mass flux of the water production exhibits a similar trend found in the temperature results of Fig. 2.7(a) because of the mass transfer and vapor pressure relation in a logarithmic function [Eq. (2.21)] and the saturation pressure and temperature relation in an exponential function [Antoine relation Eq. (2.27)]. 
(a)

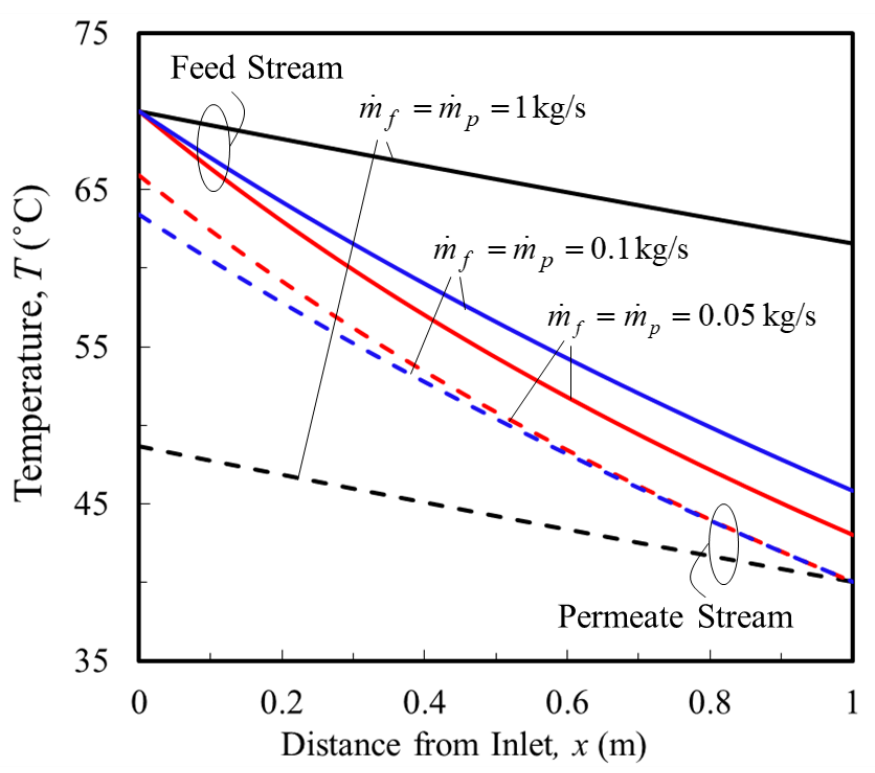

(b)

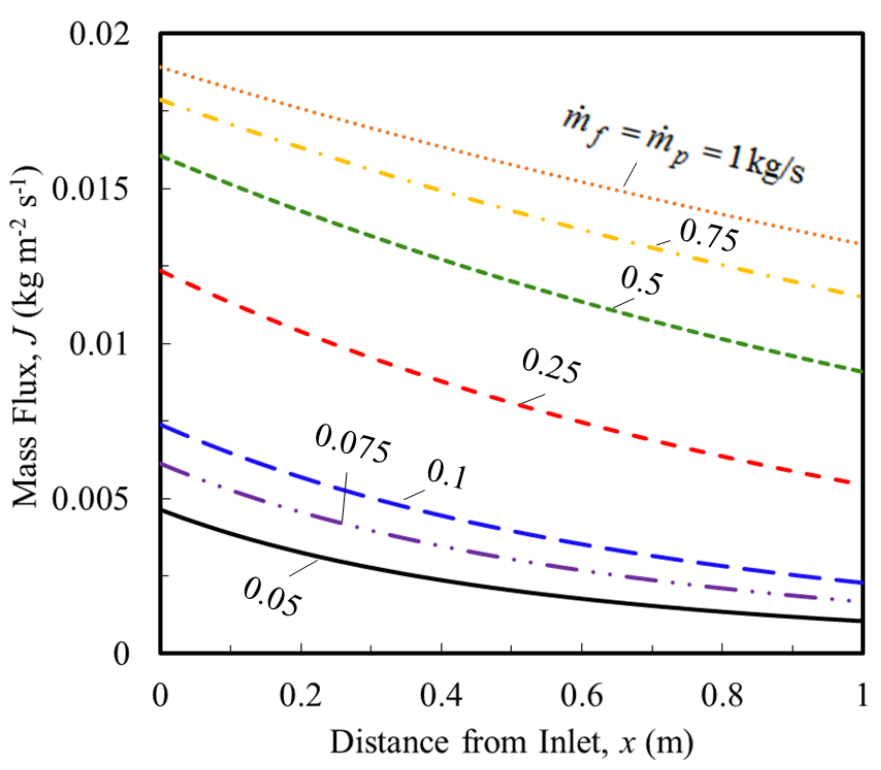

Figure 2.7 (a) Effect of feed and permeate mass flow rates on the temperature profiles and (b) the mass flux of the water distillate production of a single-module DCMD system

The thermal efficiency and heat transfer rates for the conduction and phase change are shown in Fig. 2.8(a). As the feed and permeate flow rates are increased, the phase change heat transfer outpaces the conduction loss and in turn, the thermal efficiency is 
increased. Note that the thermal efficiency increases very rapidly at the low mass flow rates which can be explained by two reasons. First, the low flow rates create a smaller temperature difference between the feed and permeate streams and in turn, the smaller vapor pressure difference across the membrane as shown in Fig. 2.7. Secondly, the higher ratio of the mass flux for water production to the mass flow rate [Eq. (2.6)] rapidly increases the concentration in the feed channel. Figure 2.8(b) shows that the water production increases as the flow feed and permeate mass flow rates increase. Higher flow rates of the feed and permeate streams are always desirable to increase the water production and thermal efficiency. However, there would be a penalty in the increased pumping power due to the increased pressure drop in the DCMD system with the higher feed and permeate flow rates. According to the Darcy-Weisbach correlation, the pumping power increases by the square of the respective mass flow rate of the feed and permeate streams. 
(a)

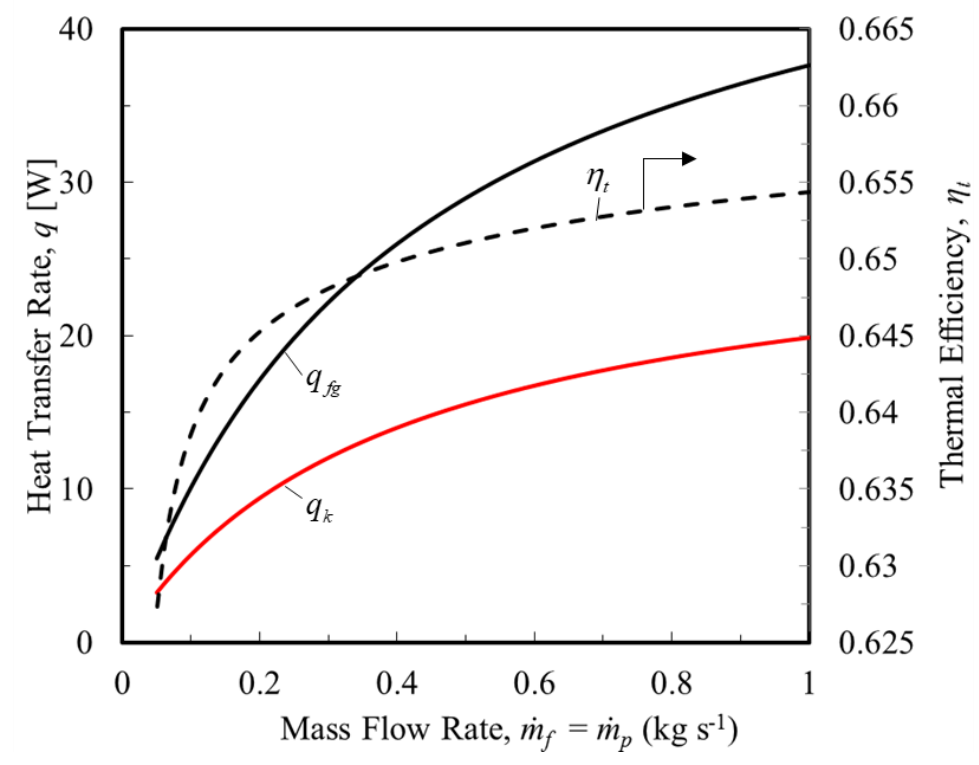

(b)

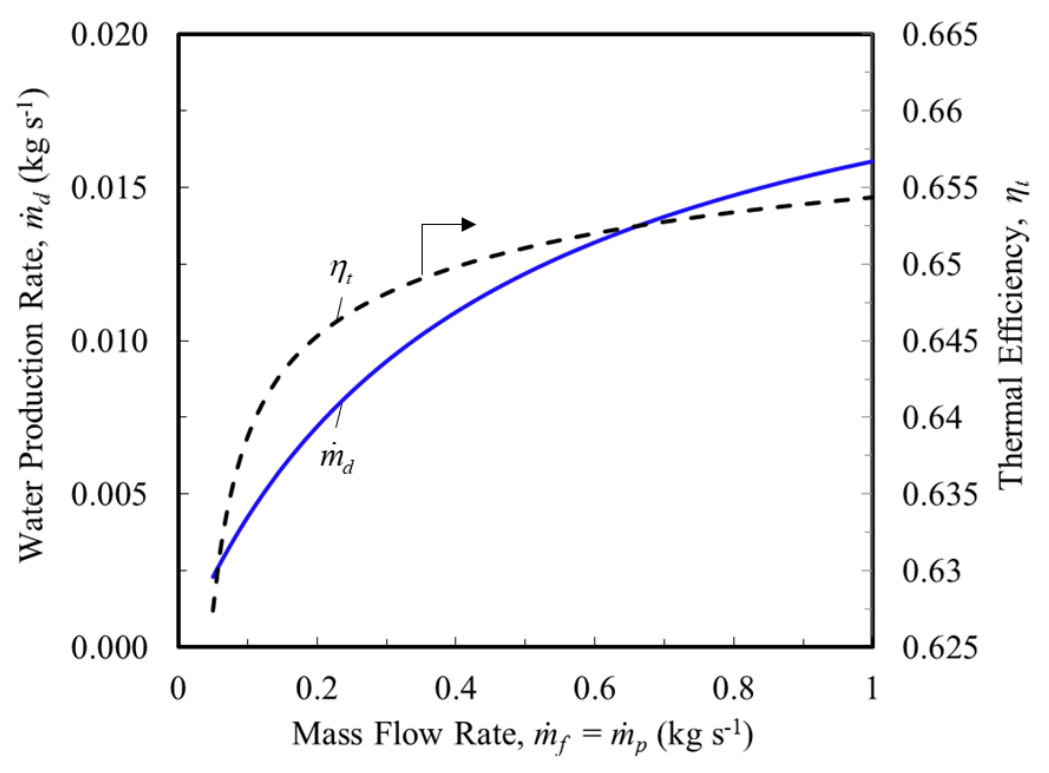

Figure 2.8 Effects of feed and permeate mass flow rates on (a) the heat transfer rates and thermal efficiency and (b) total water distillate production of a single-module DCMD system

\subsubsection{Effect of Feed and Permeate Inlet Temperature}

The effect of the inlet temperature of the feed stream on the water production is shown in Fig. 2.9 The permeate inlet temperature was kept at $40^{\circ} \mathrm{C}$ as the baseline 
condition, while the feed inlet temperature was varied from 45 to $70^{\circ} \mathrm{C}$. Figure 2.9 shows that the mass flux quickly decreased as the feed inlet temperature was decreased. This result is due to the rapid decrease in the vapor pressure at relatively high temperatures.

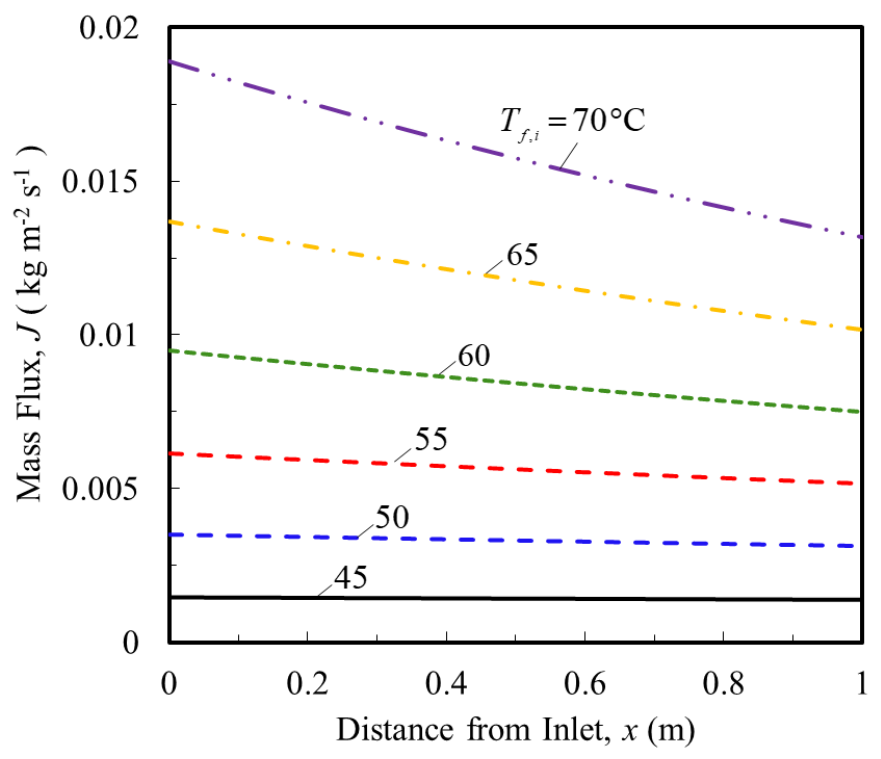

Figure 2.9 Effect of feed inlet temperature on the mass flux of the water distillate production of a single-module DCMD system

The effects of the inlet temperatures difference (ITD, $\Delta T_{i}=T_{f, i}-T_{p, i}$ ) of the feed and permeate streams into the membrane module on the water production and thermal efficiency are illustrated in Fig. 2.10. The inlet temperature difference was changed by varying the inlet temperature of one fluid stream while keeping the other fluid temperature constant. In the first case, the permeate inlet temperature $\left(T_{p, i}\right)$ was varied from 69 to $40^{\circ} \mathrm{C}$ with a fixed inlet temperature of the feed stream $\left(T_{f, i}\right)$ at $70^{\circ} \mathrm{C}$. In the second case, the feed inlet temperature $\left(T_{f, i}\right)$ was increased from 41 to $70^{\circ} \mathrm{C}$ with a fixed inlet temperature of the permeate stream $\left(T_{p, i}\right)$ at $40^{\circ} \mathrm{C}$. It is observed in Fig. 2.10 that the water production is 
increased with increasing ITD and the first case where the feed water temperature is higher, produced more efficiently water than the second case where the feed water temperature is lower. These results are attributed to the fact that conduction heat loss increases linearly with the temperature difference between fluid streams but the vapor pressure changes (phase change heat transfer) exponentially with the fluid temperature, especially at high temperatures. The optimum ITD for a maximum thermal efficiency exists around $6^{\circ} \mathrm{C}$ in the first case where the permeate water temperature and therefore the vapor pressure (phase change heat transfer) are decreased. In the second case, however, the thermal efficiency is increased continuously since the phase change heat transfer always outpaces the conduction heat loss, as the feed temperature increases. The different behaviors of the conduction and phase change heat transfers provide a useful insight into the temperature control of the fluid temperatures. To sum, it is always desirable to keep the ITD (e.g., by decreasing the permeate water temperature as in the first case and increasing the feed water temperature as in the second case) as high as possible for high thermal efficiency and more water production, only if the permeate water temperature is relatively high so that it needs to be decreased. Figure 2.10 also explains how greatly water production would be affected by the thermal performance of the heat sink heat exchanger [HX2 in Fig. 1(a)] in an attempt to keep the permeate temperature low. If the heat sink (e.g., ambient air) temperature is very high that the cooling is not sufficient, the permeate inlet temperature rises even higher resulting in a reduction of the water production. 


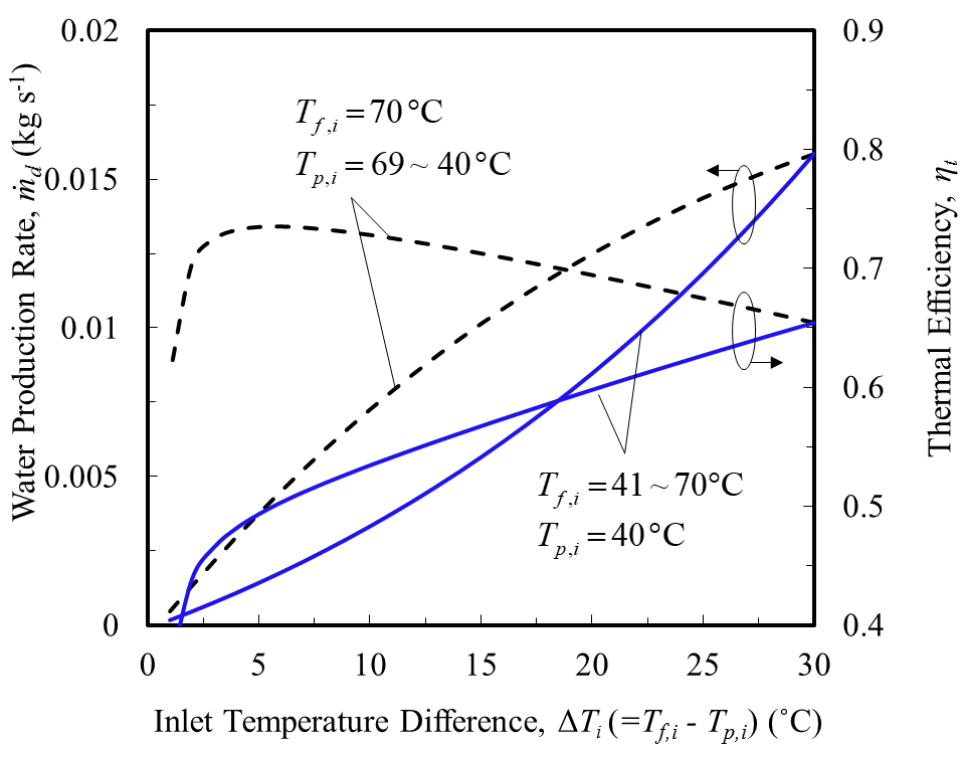

Figure 2.10 Variations of total water distillate production and thermal efficiency of a single-module DCMD system with varying inlet temperature difference of feed and permeate streams

\subsubsection{Parallel Arrangement of Multiple Direct Contact Membrane Distillation Modules}

A second DCMD system consisting of multiple membrane modules in a parallel arrangement [Fig. 2.1(b)] was analyzed under the condition that a fixed amount of energy supply $\left(q_{\text {in }}\right)$ of $672 \mathrm{~kW}$ was utilized from the diesel engine coolant and the results are presented in Fig. 2.11. The baseline operating conditions listed in Table 2.1 were used in the analysis, except the membrane length. Figure 2.11(a) shows the variations in the heat transfer area $\left(A_{H X}\right)$ of $\mathrm{HX} 1$, the total membrane surface area $\left(A_{m}\right)$, and the number of the DCMD modules $\left(N_{m}\right)$ by varying the length $\left(L_{m}\right)$ of the DCMD modules. A shorter module produces more water because it operates at a higher feed water temperature. As a result, the high feed water temperature enters HX1 causing a small temperature difference between the feed water and engine coolant and requiring a large heat transfer area $\left(A_{H X}\right)$ to exchange the fixed amount of energy supply in HX1. Since a shorter DCMD module 
provides a smaller membrane area $\left(A_{m}\right)$, a greater number $\left(N_{m}\right)$ of the short membrane modules are required for water production. Although it is common to increase the heat transfer area $\left(A_{H X}\right)$ by increasing the number $\left(N_{H X}\right)$ of the flow channels in HX1, since the heat transfer coefficient is decreased due to the reduced Reynolds number, it is more desirable to choose a reasonably long membrane (e.g., $L_{m}=0.5 \mathrm{~m}$ ) which would also lower the hardware cost associated with more modules with shorter membrane systems.

Figure 2.11(b) shows the variations in the total water production $\left(\dot{m}_{d}\right)$, mass flux $(J)$ of the DCMD system and the heat exchanger effectiveness $\left(\varepsilon_{H X}\right)$ of HX1 with respect to the length of the DCMD modules. As the membrane module gets longer, the water production (mass flux) slowly decreases, despite the increase in total membrane surface area $\left(A_{m}\right)$ as shown in Fig. 2.11(a). This is due to the heat conduction loss across the membrane which increases linearly with the membrane surface area. Thus, a longer membrane loses more heat resulting in less water production and lower thermal efficiency. The heat exchanger effectiveness of HX1 decreases for the longer DCMD module system because the inlet temperature difference between the feed (cold) and engine coolant (hot) in HX1 increases. Therefore, the higher heat exchanger effectiveness is another reason to design a DCMD system with a greater number of short membrane modules to achieve higher water production, thermal efficiency, and heat exchanger effectiveness. 
(a)

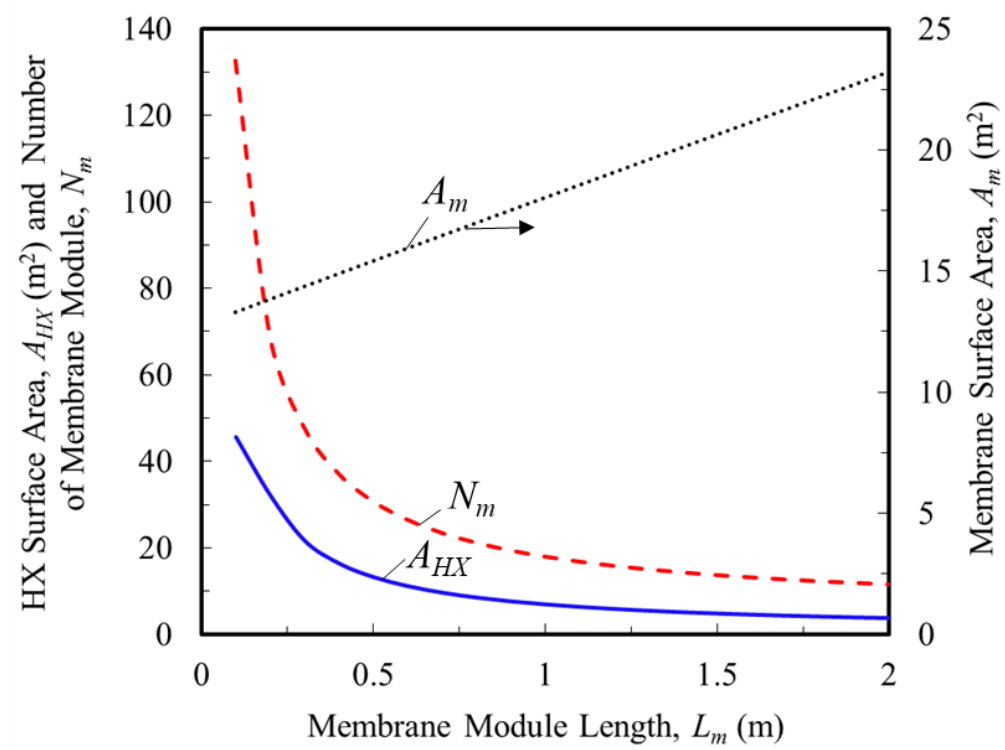

(b)

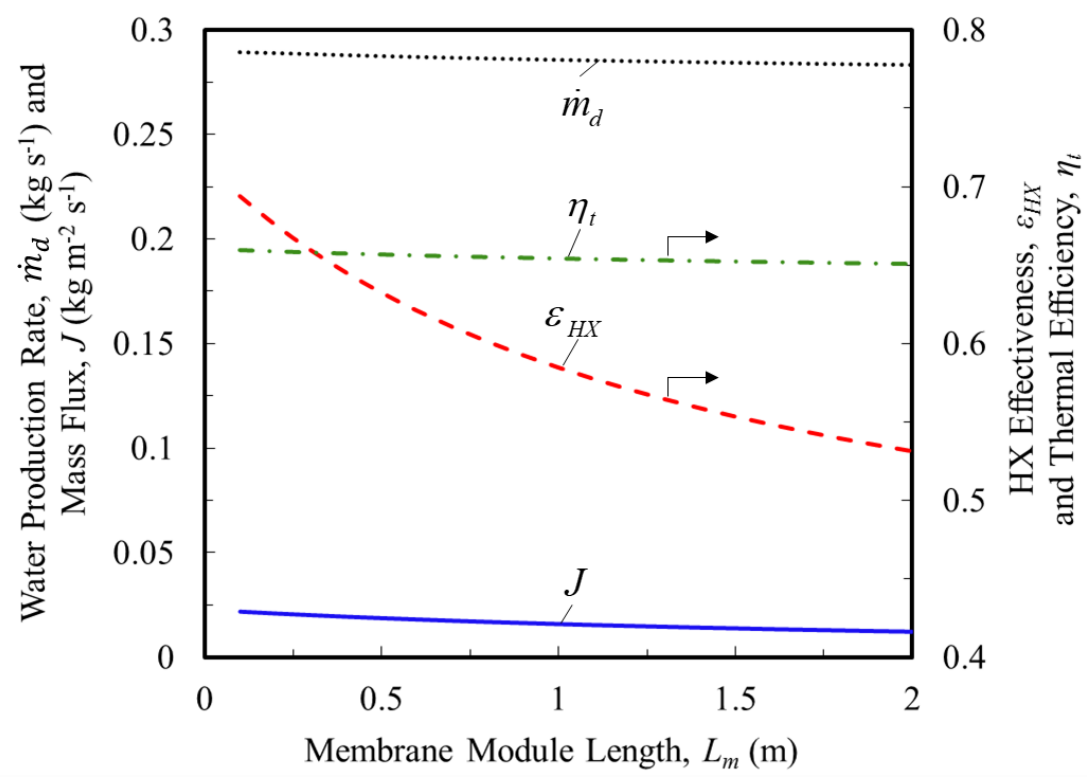

Figure 2.11 (a) Variations of heat transfer area of the heat source heat exchanger (HX1), number of membrane modules and total membrane area of a multiple-module DCMD system with varying membrane module length. (b) Variations of total water distillate production, mass flux, and thermal efficiency and effectiveness of the heat source heat exchanger (HX1) of a multiple-module DCMD system with varying membrane module length. 


\subsection{SUMMARY}

A Direct Contact Membrane Distillation (DCMD) system running on the waste heat from a diesel generator was numerically analyzed using one-dimensional energy and mass analysis. The results show that a greater number of shorter membrane modules connected in parallel improve water production and thermal efficiency of the DCMD system, and effectiveness of the heat source heat exchanger. It was also found that the higher feed water temperature, liquid flow rates, and greater temperature difference between the feed and permeate streams are desirable to increase DCMD water production and thermal efficiency. 


\section{CHAPTER 3: HEAT AND MASS TRANSFER IN DIRECT CONTACT MEMBRANE DISTILLATION}

In this section, a two-dimensional CFD model was developed to study the effects of variable operating conditions such as temperature, flow rate, flow arrangement and salinity on the distilled water production and thermal performance of a DCMD system. The CFD model has taken a transmembrane mass flux and conduction and phase change heat transfer at the permeable membrane walls into consideration. This comprehensive model is accomplished to accurately analyze the velocity, temperature and concentration boundary layer formation (polarization effect) on the membrane surfaces. Experimental data collected from a DCMD system has validated the numerical results.

\subsection{NUMERICAL METHOD}

ANSYS FLUENT, a commercial package, is used to develop a Computational Fluid Dynamics (CFD) model to simulate the heat and mass transfer in the DCMD. The DCMD system consists of two flow channels for feed and permeate streams separated by a nanoporous (permeable) membrane. Figure 3.1(a) illustrates the two-dimensional computational domain in the $x$ - and $y$-directions used in the CFD analysis. The flow in the membrane channels was assumed to be symmetric in the $z$-direction because of a large aspect ratio of the membrane channels $\left(W_{m}>>H_{m}\right)$.

The MD module consists of two acrylic plates that compress and seal the layers of mesh screen spacer and membrane. Each compression plate features a recessed surface to create a flow channel whose boundary is sealed with an O-ring and channel walls formed by the compression plate and the membrane. The screen spacers are made of polypropylene 
and have longitudinal and transverse filaments woven in a rectangular pattern forming a double-layer structure. The longitudinal filaments are oriented along the flow direction and located on the permeable membrane side, whereas the transverse filaments are orthogonal to the flow direction and located on the side of the impermeable channel wall [Figs. 3.1(bc)]. After clamping the compression plates, the screen filaments were deformed slightly. It was found from a direct measurement that the deformed filament had a width of $2.1 \mathrm{~mm}$ and a height of $0.9 \mathrm{~mm}$ and the center-to-center spacing $(S)$ between the screen filaments of $10 \mathrm{~mm}$ as shown in Fig. 3.1(b).

The two-dimensional computational domain as shown in Fig. 3.1(b) was meshed to create finer meshes near solid walls using high skewness using a size function [Fig. 3.1(c)]. The quality of the mesh was checked for a dependency of the results of CFD analysis on different mesh sizes by comparing the results of water production rate and pressure drop in the channel flows. Finer meshes were used for the flow regions adjacent to solid walls such as the membrane and channel walls. Considering the computational time and accuracy, a mesh system consisting of 475,000 elements was used for the CFD analysis as shown in Fig. 3.1(c). The mesh lines in green color are too fine to show visibly and such fine meshes are necessary for the accuracy in the simulation.

The flow, heat and mass transfers within the feed and permeate channels are governed by the conservation laws of mass, momentum, energy, and concentration. The concentration equation was solved for only the feed channel. The governing equations for mass, momentum, energy, and concentration can be found in ANSYS Fluent [30].

The flow in the membrane channels filled with a mesh spacer is turbulent. The SpalartAllmaras (SA) turbulence model used for this CFD simulation takes streamline curvature 
and flow rotation effects into account which are necessary to analyze the complex flow in the MD channels. The results from the SA model [31] were compared to those from another common turbulence model like the Shear Stress Transport (SST) $k$ - $\omega$ turbulence model and found to be in good agreement. The SIMPLE algorithm was used for coupled pressurevelocity equations, and a second-order accuracy was employed for the spatial discretization scheme. Convergence criteria were set to $1 \times 10^{-12}$ for the residuals of continuity, velocity and concentration components and energy equations. The governing equations for mass, momentum, energy, and concentration in tensor form are given by

$$
\begin{aligned}
& \frac{\partial\left(\rho U_{i}\right)}{\partial x_{i}}=0 \quad, i=1,2, \\
& \rho U_{j} \frac{\partial U_{i}}{\partial x_{j}}=-\frac{\partial p}{\partial x_{i}}+\frac{\partial p}{\partial x_{j}}\left(\mu \frac{\partial U_{i}}{\partial x_{j}}\right)+\frac{\partial\left(-\rho \bar{u}_{i}^{\prime} \bar{u}_{j}^{\prime}\right)}{\partial x_{j}}, i, j=1,2 \\
& -\rho \bar{u}_{i}^{\prime} \vec{u}_{j}=\mu_{t}\left(\frac{\partial U_{i}}{\partial x_{j}}+\frac{\partial U_{j}}{\partial x_{i}}\right), i, j=1,2, \\
& \frac{\partial}{\partial t}(\rho \tilde{v})+\frac{\partial}{\partial x_{i}}\left(\rho \tilde{v} U_{i}\right)=G_{v}+\frac{3}{2}\left[\frac{\partial}{\partial x_{j}}\left\{(\mu+\rho \tilde{v}) \frac{\partial \tilde{v}}{\partial x_{j}}\right\}+0.622 \rho\left(\frac{\partial \tilde{v}}{\partial x_{j}}\right)^{2}\right]-D_{v}, i, j=1,2, \\
& \mu_{t}=\rho \tilde{v} f_{v 1}, \\
& \frac{\partial}{\partial t}(\rho E)+\frac{\partial}{\partial x_{i}}\left[U_{i}(\rho E+p)\right]=\frac{\partial}{\partial x_{j}}\left(k \frac{\partial T}{\partial x_{j}}+U_{i} \tau_{i j, e}\right), \\
& \frac{\partial\left(U_{i} C\right)}{\partial x_{i}}=-\frac{\partial}{\partial x_{j}}\left[\left(D+D_{t}\right) \frac{\partial C}{\partial x_{j}}\right], i, j=1,2
\end{aligned}
$$


the prime sign shows the fluctuation component of the variables and all variables in Eqs. (3.1-7) are time-averaged. Velocity is expressed as $u_{i}\left(x_{k}, t\right)=U_{i}\left(x_{k}\right)+u_{i}^{\prime}\left(x_{k}, t\right)$ which the time averaged velocity is defined as $U_{i}\left(x_{k}\right)=\lim _{T \rightarrow \infty} \frac{1}{T} \int_{0}^{T} u\left(x_{k}, t\right) d t . \tilde{v}$ is called the turbulent kinematic viscosity and Eq. (3.4) shows the governing equation for the transported variable $(\tilde{v})$ in the Spalart-Allmaras model. $G_{v}$ and $D_{v}$ are the production and destruction of turbulent viscosity, respectively. Turbulent viscosity, $\mu_{t}$ is calculated based on turbulent kinematic viscosity $(\tilde{v})$ and viscous damping function $\left(f_{v 1}\right)$ regarding Eq. (3.5). Turbulent heat transport is determined by Eq. (3.6) where $k$ is the thermal conductivity, $E$ is the total energy and $\tau_{i j, e}$ is the effective deviatoric stress tensor. $D_{t}$ is the coefficient of diffusion describing the strength of turbulence. Further details about the Spalart-Allmaras model can be found in [31].

A velocity boundary condition was used at the inlets of the feed and permeate channels and a pressure boundary condition was used at the outlets of the channels. The boundary conditions used for the simulation are marked in the simulation domain as illustrated in Fig. 3.1(c) and the equations are listed below.

(i) Feed or permeate channel inlets:

$$
\left\{\begin{array}{l}
u=U_{f, i} \text { or } U_{p, i}, \\
v=0, \\
T=T_{f, i} \text { or } T_{p, i}, \\
C=C_{f, i} \text { or } 0 .
\end{array},\right.
$$

(ii) Feed or permeate channel outlets: 


$$
p=p_{f, o} \text { or } p_{p, o} \text {. }
$$

(iii) Impermeable walls of feed and permeate channels:

$$
\left\{\begin{array}{l}
u=0, \\
v=0, \\
\frac{\partial T}{\partial y}=0, \\
\frac{\partial C}{\partial y}=0 .
\end{array}\right.
$$

(iv) Feed side of permeable membrane:

$$
\left\{\begin{array}{l}
u=0 \\
v=-\frac{J}{\rho} \\
q^{\prime \prime}=-\left(q_{f g}^{\prime \prime}+q_{k}^{\prime \prime}\right), \\
\frac{\partial C}{\partial y}=-\frac{J}{\rho D_{s}} .
\end{array}\right.
$$

(v) Permeate side of permeable membrane:

$$
\left\{\begin{array}{l}
u=0, \\
v=\frac{J}{\rho}, \\
q^{\prime \prime}=q_{f g}^{\prime \prime}+q_{k}^{\prime \prime}, \\
\frac{\partial C}{\partial y}=0 .
\end{array}\right.
$$

Here, $q^{\prime \prime}$ is the total heat flux which is a sum of (i) the phase change heat transfer (evaporation and condensation) through the membrane, $q_{f g}^{\prime \prime}\left(=J h_{f g}\right)$, and (ii) the conduction heat transfer through the membrane, $q_{k}^{\prime \prime}\left[=k_{m, e}\left(T_{f, m}-T_{p, m}\right) / \delta_{m}\right] . J$ is the transmembrane mass flux, and $\delta_{m}$ is the thickness of the membrane. 
(a)

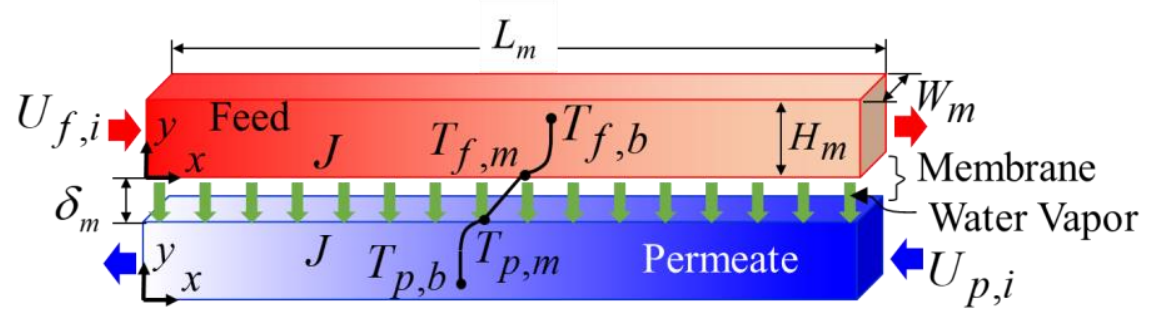

(b)

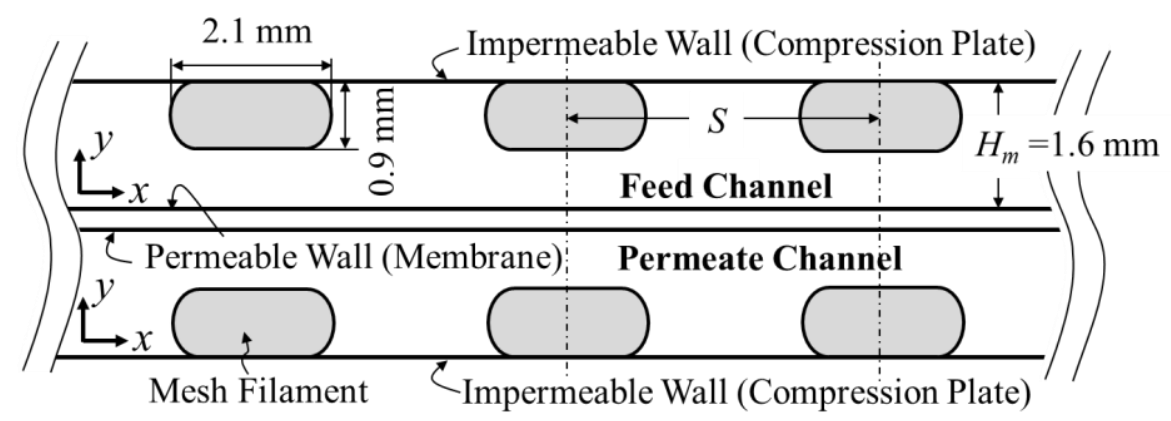

(c)

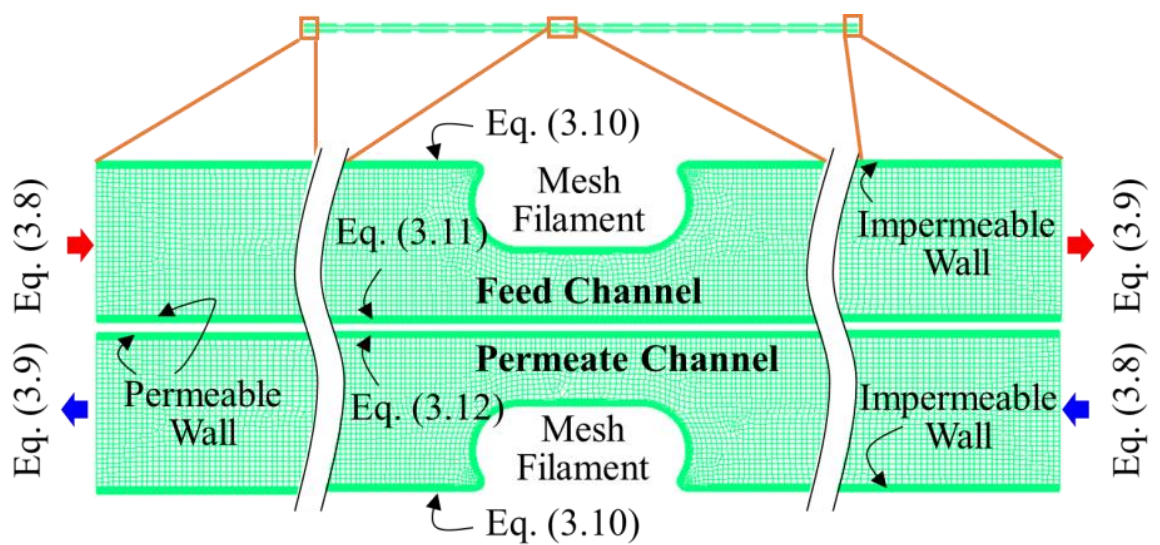

Figure 3.1 (a) Computational domain, (b) geometries and dimensions of the filaments of the mesh screen spacer and the spacing between the filaments of the DCMD system and (c) boundary conditions used for CFD analysis 
The effective thermal conductivity of the membrane, $k_{m, e}$ can be determined by considering the conduction heat transfers through the solid and gas phases of the membrane and is calculated by

$$
k_{m, e}=\left(1-\varepsilon_{m}\right) k_{m}+\varepsilon_{m} k_{g},
$$

where, $\varepsilon_{m}$ and $k_{m}$, are the porosity and the bulk thermal conductivity of the membrane material, respectively. $k_{g}$ is the thermal conductivity of water vapor.

The fluid velocity normal to the membrane walls [ $v$ in Eqs. (3.4) and (3.5)] is determined from the transmembrane mass flux, $J$ [Eq. (3.7)]. The wall heat flux $\left(q^{\prime \prime}\right)$ applied to the membrane surfaces includes a latent heat $\left(q_{f g}^{\prime \prime}\right)$ and a conduction heat transfer $\left(q_{k}^{\prime \prime}\right)$. The transmembrane mass flux, wall heat flux and concentration gradients for the permeable membrane surfaces are calculated using a User Defined Function (UDF) embedded in the FLUENT.

The water vapor transfer through the nanochannel network in the membrane can be simulated as gas transport in porous media known as the Dusty Gas Model (DGM) which is characterized by four available mechanisms: surface diffusion, molecular diffusion, Knudsen diffusion, and viscous flow. In the DCMD system, the surface diffusion and viscous flow are negligible [25]. In the Knudsen diffusion regime, the gas density is so low that a gas molecule can travel without colliding with other gas molecules (i.e., long mean free path) or the nanochannels are so narrow that the number of molecule-wall collisions is much more than molecule-molecule collisions. In contrast, in the molecular-diffusion regime, the number of molecule-molecule collisions dominate molecule-wall collisions. 
Since the mean free path of the water molecules flow is comparable to the pore size of MD membrane materials, the mass transport through the membrane is at a transition regime. It means that the frequency of the collisions between the water molecules and pore wall of the membrane (Knudsen diffusion) is comparable to the collisions between the water molecules (molecular diffusion). Thus, the series combination of the molecular and Knudsen diffusion is used to determine the water mass flux as follows [25]

$$
\begin{aligned}
& J=\frac{\left(\varepsilon_{m} / \tau_{m}\right) p_{T} D_{w v-a} M_{w}}{(1-\alpha) \delta_{m} R T_{m}} \ln \left\{\left\{\frac{D_{k n}\left[p_{T}-(1-\alpha) p_{v . p}\right]+\left(\varepsilon_{m} / \tau_{m}\right) p_{T} D_{w v-a}}{D_{k n}\left[p_{T}-(1-\alpha) p_{v . f}\right]+\left(\varepsilon_{m} / \tau_{m}\right) p_{T} D_{w v-a}}\right\}\right\}, \\
& D_{K n}=\frac{4 \varepsilon_{m}}{3 \tau_{m}} d_{p} \sqrt{\frac{R T_{m}}{2 \pi M_{w}}}, \\
& p_{T} D_{w-a}=1.895 \times 10^{-5} T_{m}^{2.072} \\
& \alpha=\frac{M_{w}}{M_{a}}
\end{aligned}
$$

where $\tau_{m}, \varepsilon_{m}, \delta_{m}$, and $d_{p}$ are the tortuosity, porosity, thickness, and pore diameter of the membrane, respectively, and their values are listed in Table 3.1. $D_{K n}$ is the diffusivity for Knudsen diffusion. $p_{T} D_{w v-a}$ is a product of the total pressure $\left(p_{T}\right)$ and water-vapor diffusivity $\left(D_{w v-a}\right)$ in the air and has the unit of $\left[\mathrm{Pa}-\mathrm{m}^{2} / \mathrm{s}\right] . T_{m}$ is the membrane temperature in Kelvin and determined by the average of the local feed and permeate temperatures $\left(T_{f, m}\right.$, $\left.T_{p, m}\right)$ on the membrane surfaces. $M_{w}$ and $M_{a}$ are the molecular masses of water and air, respectively. 
Table 3.1 Dimensional and thermophysical properties of the membrane used in the DCMD system

\begin{tabular}{ccccccc}
\hline \multirow{2}{*}{ Membrane type } & $\begin{array}{c}\delta_{m} \\
{[\mathrm{~m}]}\end{array}$ & $\begin{array}{c}d_{p} \\
{[\mathrm{~m}]}\end{array}$ & $\begin{array}{c}\varepsilon_{m} \\
{[-]}\end{array}$ & $\begin{array}{c}\tau_{m} \\
{[-]}\end{array}$ & $\begin{array}{c}k_{m} \\
{[\mathrm{~W} / \mathrm{m}-\mathrm{K}]}\end{array}$ & $\begin{array}{c}\theta_{c} \\
{\left[{ }^{\circ}\right]}\end{array}$ \\
\hline QM022 & $84 \times 10^{-6}$ & $0.36 \times 10^{-6}$ & 0.62 & 2.34 & 0.23 & 127 \\
\hline
\end{tabular}

In Eq. (3.7), the vapor pressures $\left(p_{v, f}, p_{v, p}\right)$ of the feed and permeate streams are evaluated at their local fluid temperatures on the membrane surfaces. For non-ideal binary mixtures containing a non-volatile solute $(\mathrm{NaCl})$, the vapor pressure can be determined by

$$
p_{v}=(1-M) p_{s a t},
$$

where the water activity of $\mathrm{NaCl}$ solutions, $a_{w}$, is a function of the salt concentration and determined by [26]

$$
a_{w}=1-0.5 M-10 M^{2}
$$

where $M$ is the mole fraction of $\mathrm{NaCl}$ solutions. The pure water saturation pressure $\left(p_{\text {sat }}\right)$ in the unit of Pascal can be determined by the Antoine equation [27] below

$$
p_{\text {sat }}=\exp \left(23.1964-\frac{3816.44}{T-46.13}\right)
$$

where $T$ is the local fluid temperature in Kelvin.

Finally, the water production rate is given by

$$
\dot{m}_{w}=J A_{m}
$$

where $A_{m}$ is the membrane area $\left(A_{m}=W_{m} L_{m}\right)$.

Figure 3.2 shows a thermal circuit showing the thermal resistances and heat transfers in the membrane module. The heat transfers are (i) convection heat transfer ( 
$\left.q_{f}^{\prime \prime}, q_{p}^{\prime \prime}\right)$ between the feed and permeate streams, and the membrane surfaces; (ii) phase change heat transfer $\left(q_{f g}^{\prime \prime}\right)$ of evaporation on the feed membrane surface and condensation on the permeate membrane surface, which are carried by the water vapor flow through the membrane (advection); and (iv) conduction heat transfer $\left(q_{k}^{\prime \prime}\right)$ across the membrane, a parasitic heat loss. Note that the advection and conduction heat transfers occur in parallel (Fig. 3.2).

The thermal resistances $\left(R^{\prime \prime}\right)$ based on a heat flux are used in the thermal circuit shown in Fig. 3.2 The total thermal resistance of the thermal circuit is given by

$$
R_{T}^{\prime \prime}=R_{f}^{\prime \prime}+\left(R_{k}^{\prime \prime-1}+R_{f g}^{\prime \prime-1}\right)^{-1}+R_{f}^{\prime \prime}
$$

where,

$$
\begin{aligned}
& R_{f}^{\prime \prime}=\frac{\left(T_{f, b}-T_{f, m}\right)}{q_{f}^{\prime \prime}}, \\
& R_{f g}^{\prime \prime}=\frac{\left(T_{f, m}-T_{p, m}\right)}{q_{f g}^{\prime \prime}}, \\
& R_{k}^{\prime \prime}=\frac{\left(T_{f, m}-T_{p . m}\right)}{q_{k}^{\prime \prime}}, \\
& R_{p}^{\prime \prime}=\frac{\left(T_{p, m}-T_{p, b}\right)}{q_{p}^{\prime \prime}},
\end{aligned}
$$

where $R_{f}^{\prime \prime}$ and $R_{p}^{\prime \prime}$ are the convective thermal resistances in the feed and permeate channels, respectively. $R_{k}^{\prime \prime}$ is the conduction thermal resistance across the membrane and is calculated by $R_{k}^{\prime \prime}=\delta_{m} / k_{m, e} . R_{f g}^{\prime \prime}$ is the thermal resistance of the phase change heat transfer which is 
carried by the water vapor flow through the membrane. $R_{k, f_{g}}^{\prime \prime}$ is the sum of the conduction thermal resistance and phase change thermal resistance connected in parallel, i.e., $R_{k, f g}^{\prime \prime}=\left(R_{k}^{\prime \prime-1}+R_{f g}^{\prime \prime-1}\right)^{-1}$

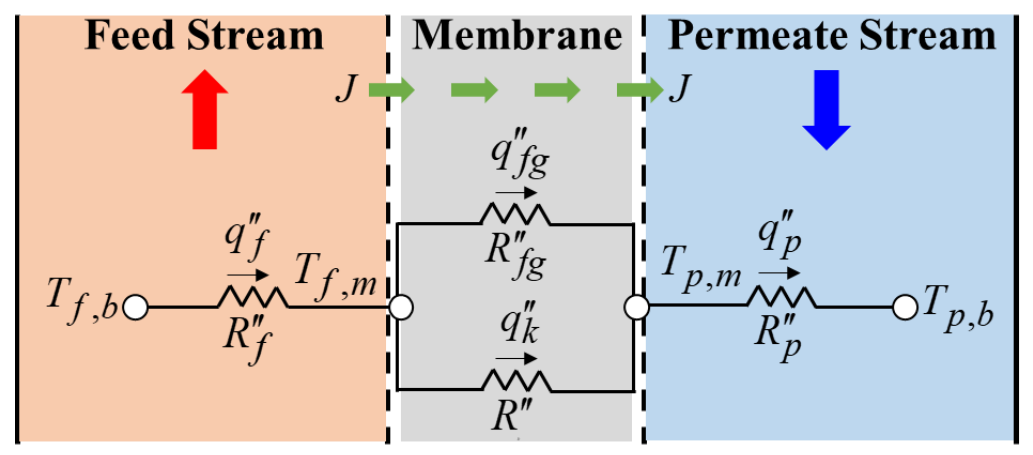

Figure 3.2 Thermal circuit diagram for heat transfers in the DCMD system

The Temperature Polarization Coefficient (TPC) is often used to measure the efficacy of the convective heat transfer in the flow channels and is given by

$$
\mathrm{TPC}=\frac{T_{f, m}-T_{p, m}}{T_{f, b}-T_{p, b}}
$$

where, $T_{f, m}$ and $T_{p, m}$ are the feed and permeate fluid temperatures on the membrane surfaces. $T_{f, b}$ and $T_{p, b}$ are the bulk fluid temperatures of the feed and permeate flows. The TPC can be expressed using thermal resistances (Fig. 3.2) and is given by

$$
\mathrm{TPC}=\frac{R_{k, f g}^{\prime \prime}}{R_{T}^{\prime \prime}}=1-\frac{R_{f}^{\prime \prime}+R_{p}^{\prime \prime}}{R_{T}^{\prime \prime}},
$$

A TPC of unity means that the convective heat transfer of the channel flows is so effective that the convective resistances $\left(R_{f}^{\prime \prime}\right.$ and $\left.R_{p}^{\prime \prime}\right)$ can be considered negligible. That is, $R_{T}^{\prime \prime}=R_{k, f g}^{\prime \prime}$ and therefore, $T_{f, m}=T_{f, b}$ and $T_{p, m}=T_{p, b}$. In this case, the membrane distillation 
is limited by the mass transport via the membrane rather than the convective heat transfer between the liquid (feed and permeate) flows and the membrane surfaces.

The Concentration Polarization Coefficient (CPC) is used to measure a lateral variation of the mass concentration in the feed channel and is given by

$$
\mathrm{CPC}=\frac{C_{f, m}}{C_{f, b}}
$$

where $C_{f, m}$ and $C_{f, b}$ are the concentrations on the membrane surface and bulk fluid.

The thermal efficiency of the DCMD system can be defined by the ratio of the phase change heat transfer to the total heat transfer across the membrane. The thermal efficiency is a barometer of the efficiency of the thermal energy utilization of membrane distillation and is given by

$$
\eta_{t}=\frac{q_{f g}}{q_{f g}+q_{k}}
$$

where,

$$
\begin{aligned}
& q_{f g}=W_{m} \int_{0}^{L_{m}} q_{f g}^{\prime \prime} d x=W_{m} \int_{0}^{L_{m}} J h_{f g} d x, \\
& q_{f g}=W_{m} \int_{0}^{L_{m}} q_{k}^{\prime \prime} d x=W_{m} \int_{0}^{L_{m}} \frac{k_{m}}{\delta_{m}}\left(T_{f}-T_{p}\right) d x .
\end{aligned}
$$

\subsection{RESULTS AND DISCUSSION}

The characteristics of distilled water production with varying inlet feed temperatures paired with the baseline inlet permeate temperature, and varying inlet permeate temperatures paired with the baseline inlet feed temperature were investigated. The parameter ranges used in this study are listed in Table 3.2, with the baseline conditions 
in parentheses. The baseline inlet feed and permeate temperatures were $60^{\circ} \mathrm{C}$ and $25^{\circ} \mathrm{C}$, respectively. For simplicity, the feed and permeate flow rates were set to be equal to each

other $\left(\dot{m}_{f}=\dot{m}_{p}\right)$, with the baseline flow rates of the feed and permeate streams at $9.7 \mathrm{~g} / \mathrm{s}$ (or $581 \mathrm{CCM}$ ). The inlet feed temperature varied from $45^{\circ} \mathrm{C}$ to $75^{\circ} \mathrm{C}$ in increments of $5^{\circ} \mathrm{C}$, while the inlet permeate temperature varied from $10^{\circ} \mathrm{C}$ to $40^{\circ} \mathrm{C}$ in increments of $5^{\circ} \mathrm{C}$.

\section{Table 3.2. Operating conditions and dimensions of the DCMD system}

\begin{tabular}{|c|c|c|c|}
\hline \multicolumn{4}{|c|}{ Operating conditions (baseline conditions) } \\
\hline $\begin{array}{c}T_{f, i} \\
{\left[{ }^{\circ} \mathrm{C}\right]}\end{array}$ & $\begin{array}{l}T_{p, i} \\
{\left[{ }^{\circ} \mathrm{C}\right]}\end{array}$ & $\begin{array}{c}\dot{m}_{f}=\dot{m}_{p} \\
{[\mathrm{~g} / \mathrm{s}]}\end{array}$ & $\begin{array}{c}C_{f, i} \\
{[\mathrm{~kg}-\mathrm{NaCl} / \mathrm{kg}]}\end{array}$ \\
\hline $\begin{array}{c}45 \sim 75 \\
(60) \\
\end{array}$ & $\begin{array}{c}10 \sim 40 \\
(25)\end{array}$ & $\begin{array}{c}4.8 \sim 14.5 \\
(9.7) \\
\end{array}$ & $\begin{array}{c}0 \sim 0.0457 \\
(0)\end{array}$ \\
\hline \multicolumn{4}{|c|}{ Dimensions of the DCMD system } \\
\hline$L_{m}[\mathrm{~mm}]$ & $W_{m}[\mathrm{~mm}]$ & $\begin{array}{c}H_{m} \\
{[\mathrm{~mm}]}\end{array}$ & $\begin{array}{c}A_{m}\left[\mathrm{~m}^{2}\right]\left(=L_{m} \times\right. \\
\left.W_{m}\right)\end{array}$ \\
\hline 150 & 95.3 & 1.6 & 0.0143 \\
\hline
\end{tabular}

\subsubsection{Baseline Results of Flow, Heat and Mass Transfers}

The CFD simulation results using the baseline conditions (Table 3.2) in a counterflow arrangement are shown in Figure 3.3. The velocity and temperature contours in the flow channels at the inlets, midsections, and outlets of the channels are shown in Figs. 3.3(a,b). Figure 3.3(a) shows the velocity contour and streamlines in the feed and permeate channels. In a counterflow arrangement, the feed stream flows from left to right in the top channel while the permeate stream flows in the opposite direction in the bottom channel. It is observed from Fig. 3.3(a) that each stream repeats an acceleration (converging flow) and a deceleration (diverging flow), as it flows through the narrow regions before and after the spacer filaments, respectively. This repetitive flow pattern throughout the 
channels results in the fluctuations in the temperature and pressure as they can be seen in the following figures. In addition, the vortexes formed downstream of the filaments further promote the flow mixing. The figure also shows that the insertion of the mesh spacer shortens the entrance region at the channel inlets due to turbulent mixing of meandering flows. Figure 3.3(b) shows the temperature contours in the flow channels. Note that the dense temperature contours at the narrow channels indicate a thinner thermal boundary layer which leads to a heat transfer enhancement.

Figure 3.3(c) shows the variations of the membrane surface temperatures in the feed and permeate channels. It is observed that the membrane temperatures fluctuate with the converging-diverging flow around the spacer filaments. The peaks of the membrane temperature $\left(T_{f, m}\right)$ on the feed side, valleys of the membrane temperature $\left(T_{p, m}\right)$ on the permeate side, and the peaks of the membrane temperature difference $\left(\Delta T_{m}\right)$ coincide with the locations of the mesh screen filament [Fig. 3.3(b)]. The conduction heat flux $\left(q_{k}^{\prime \prime}\right)$ across the membrane exhibits a similar slight fluctuation in the membrane temperature difference $\left(\Delta T_{m}\right)$ but stays relatively constant. In contrast, the latent heat flux $\left(q_{f g}^{\prime \prime}\right)$ rapidly decreases at the inlet of the feed channel, because the water vapor pressure of the feed stream exponentially decreases with decreasing temperature. This vapor pressure result is discussed more in Fig. 3.3(d). The variations of the water vapor pressure difference $\left(\Delta p_{v}\right)$ between the feed and permeate channels rapidly decreased due to the steep change in the vapor pressure of water, especially in the feed stream at higher temperatures, according to the Antoine relation in Eq. (3.20). This result is because the sensitivity of the saturation pressure is greater at higher temperatures. The changes in water mass flux $(J)$ show a trend 
similar to that of changes in pressure difference since the water mass flux is chiefly influenced by the water vapor pressure difference across the membrane.

(a)

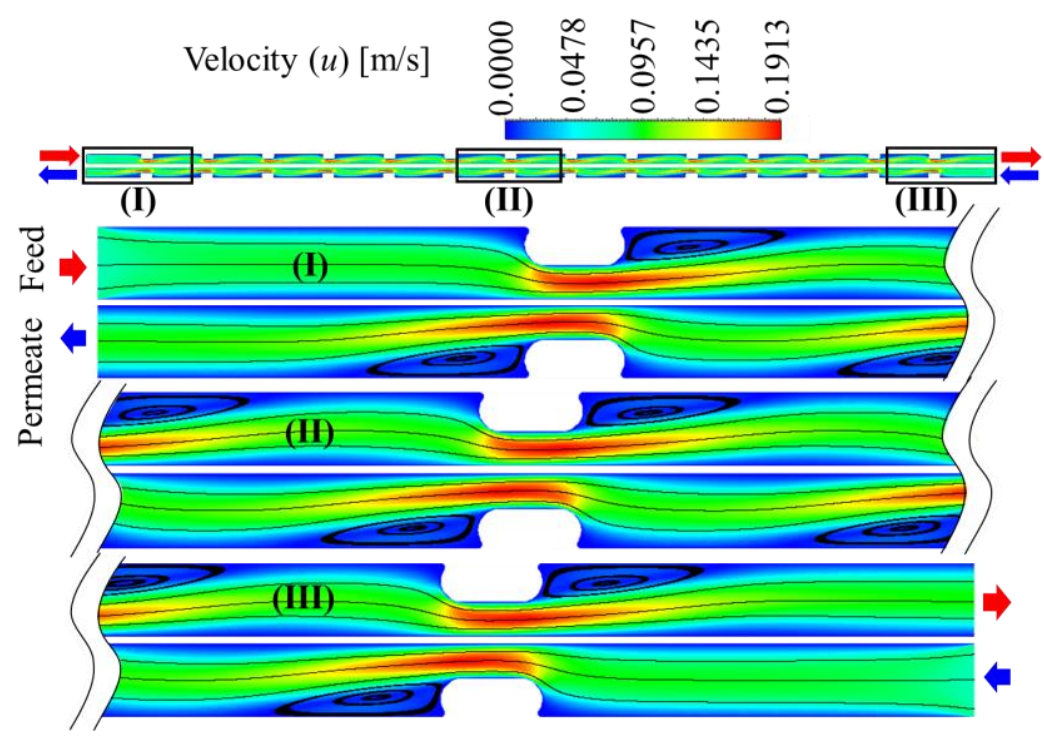

(b)

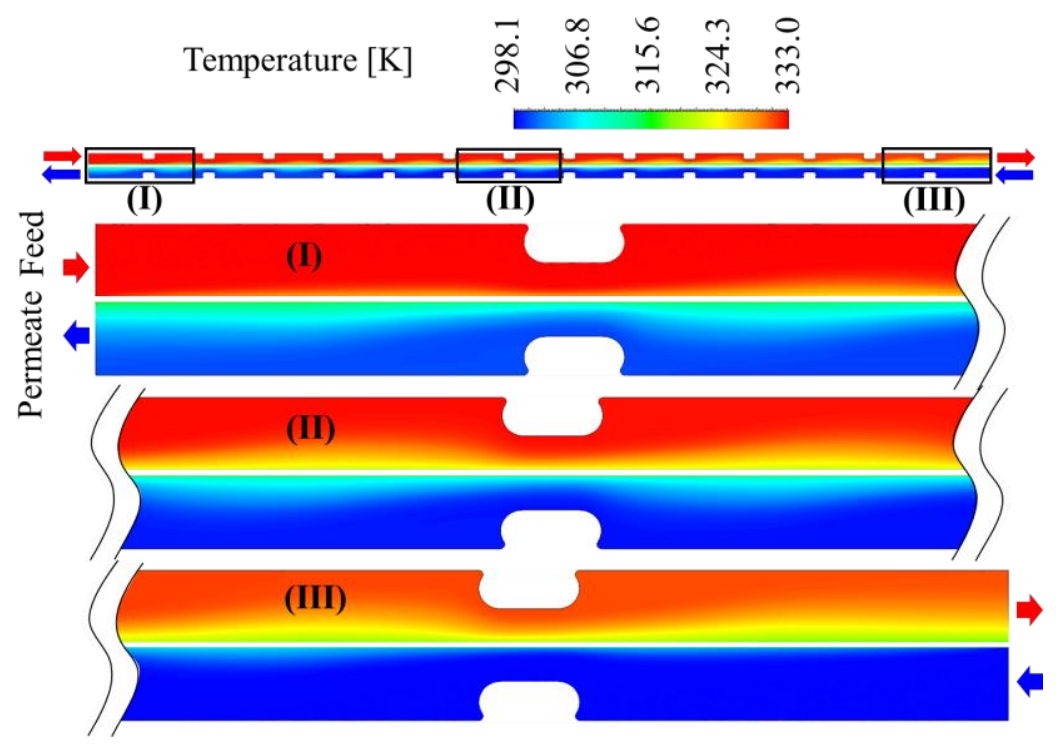


(c)

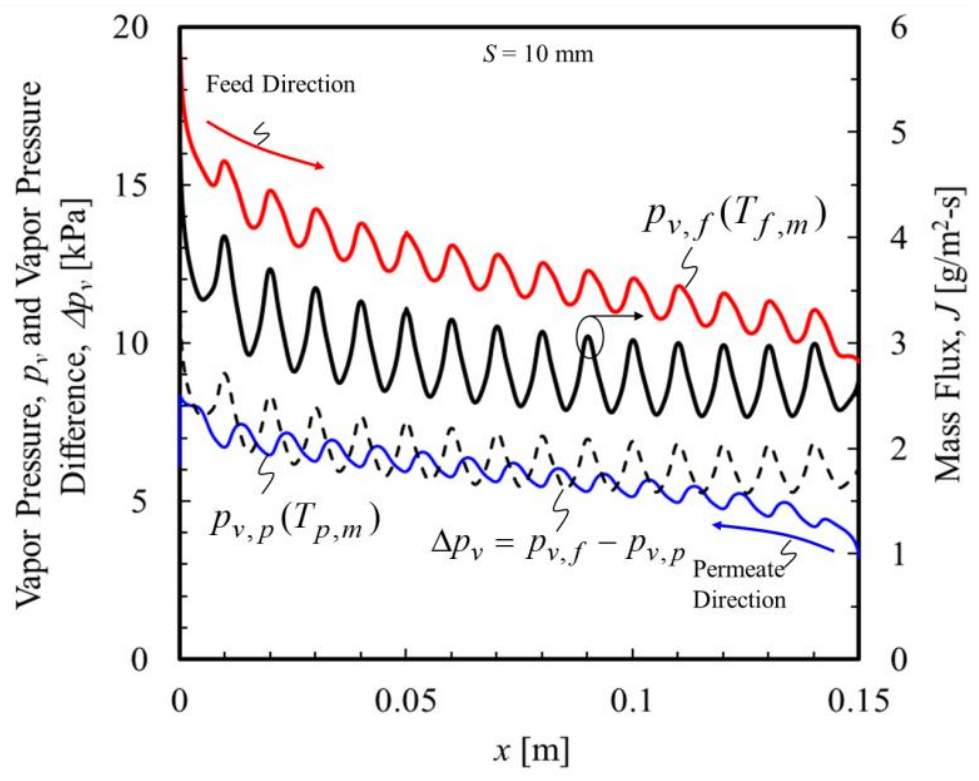

(d)

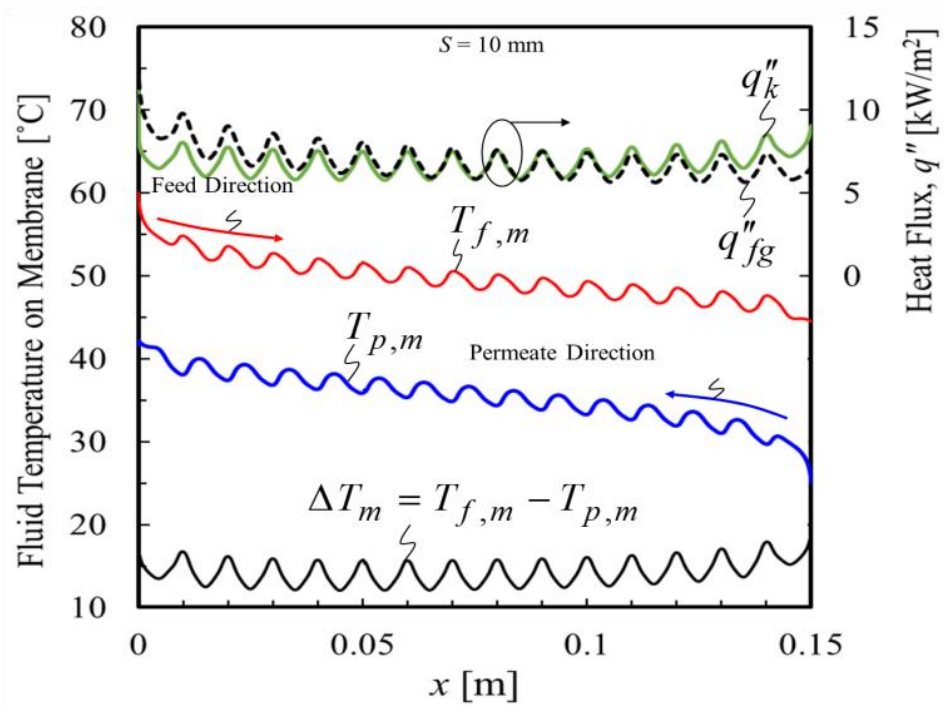

Figure 3.3 (a) velocity contour in the feed and permeate channels, (b) temperature contour in the feed and permeate channels, (c) variations of fluid temperatures of the feed and permeate channels and conduction and phase change heat fluxes, and (d) variations of the vapor pressures of the feed and permeate channels and vapor pressure difference between the feed and permeate channels and membrane mass flux 


\subsubsection{Parametric Study}

The key operational parameters of a DCMD system such as inlet temperatures and flow rates of the feed and permeate streams and salinity of the feed stream were varied to study the DCMD performance characteristics such as water production rates, heat transfer rates, and thermal efficiency.

The experimental and numerical results of feed inlet temperature variation are compared in Figure 3.4. It is interesting to observe in Fig. 3.4(a) that, with an increasing feed temperature, the water production rate exponentially increased. This is because the water vapor pressure of the feed stream and therefore, the water vapor pressure difference between the feed and permeate streams rapidly increase due to the steep increase in the vapor pressure of water in the feed stream, especially at higher temperatures as seen in Fig. 3.4(d). For the same reason, in Fig. 3.4(b), the phase change heat transfer $\left(q_{f g}\right)$ also rapidly increases with the feed water temperature. In contrast, the conduction heat transfer $\left(q_{k}\right)$ linearly increases with the feed temperature because of the linear increase in the temperature difference between the feed and permeate streams [Fig. 3.4(a)]. Since the phase change heat transfer outpaces the conduction heat transfer which is a parasitic heat loss for the MD operation, especially at higher feed temperatures, the thermal efficiency [Eq. (3.30)] increases.

The influences of the permeate inlet temperature on the water production rate and the outlet temperatures of the feed and permeate flows were investigated and the experimental and numerical results are shown in Fig. 3.5. The increasing permeate inlet temperature means a decrease in the temperature difference and therefore, the vapor pressure difference between the feed and permeate streams, which leads to a reduction in 
water production as shown in Fig. 3.5(a). The water vapor pressure tends to linearly change at lower fluid temperatures but exponentially at higher fluid temperatures [Eq. (3.20)]. Since the permeate temperature is always lower than the feed stream in the DCMD operating conditions, the impact of the change in the permeate temperature on the water production rate (phase change heat transfer) is always less profound than the change in the feed temperature. 
(a)

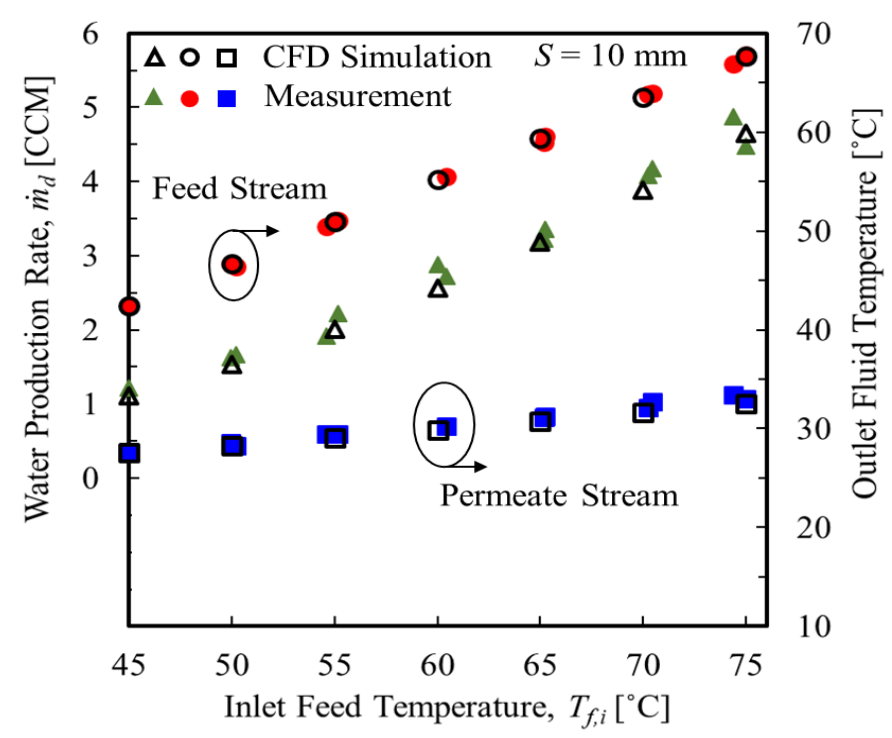

(b)

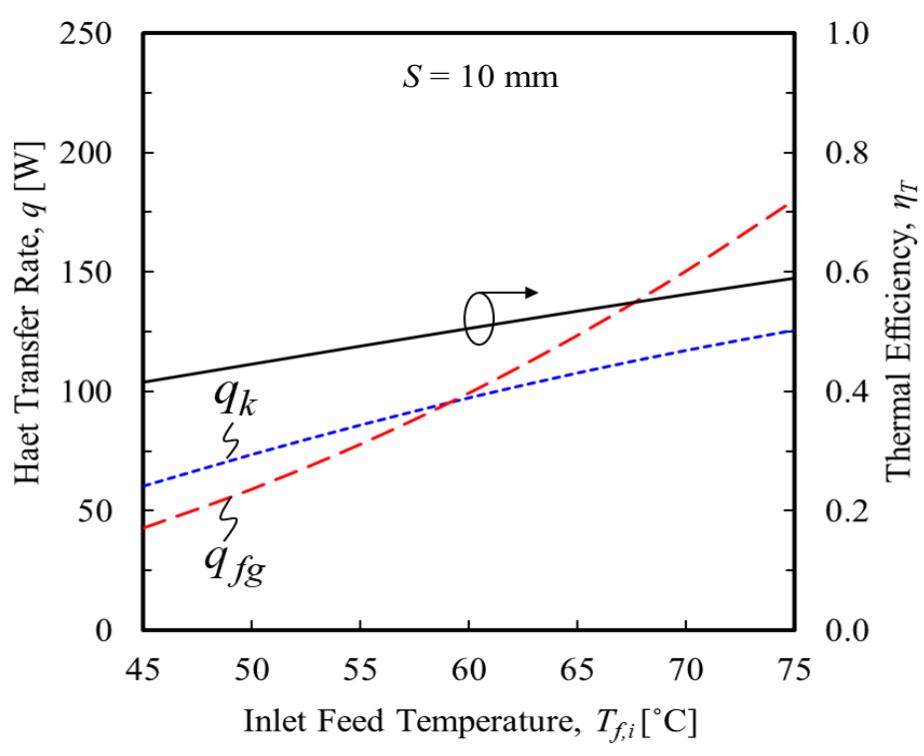

Figure 3.4 Effect of feed temperature on (a) water production rate and outlet fluid temperature and (b) conduction and phase change heat transfer rates and thermal efficiency of the DCMD system

Unlike the phase change heat transfer in a strong coupling with the feed water temperature, the conduction heat transfer is unbiased with the feed and permeate streams because it is affected by only the temperature difference between the feed and permeate 
streams, not by the magnitude of the fluid temperature. These contrasting results are manifested in Fig. 3.5(b), which shows that both the conduction and phase change heat transfer rates decrease with increasing permeate inlet temperatures, but the reduction in the conduction heat transfer decreases faster than the phase change transfer and therefore, the thermal efficiency decreases. A similar conclusion can be drawn with the results shown in Fig. 3.4(b). The differences between the computational and experimental results are about $7 \%$ for the water production rate for both varying feed and permeate inlet temperatures [Figs. 3.4(a) and 3.5(a)]. Possible sources of these errors are instrument error and assumptions in the CFD simulation such as negligible heat loss with the ambient and conduction in the acrylic plates of the MD module. 
(a)

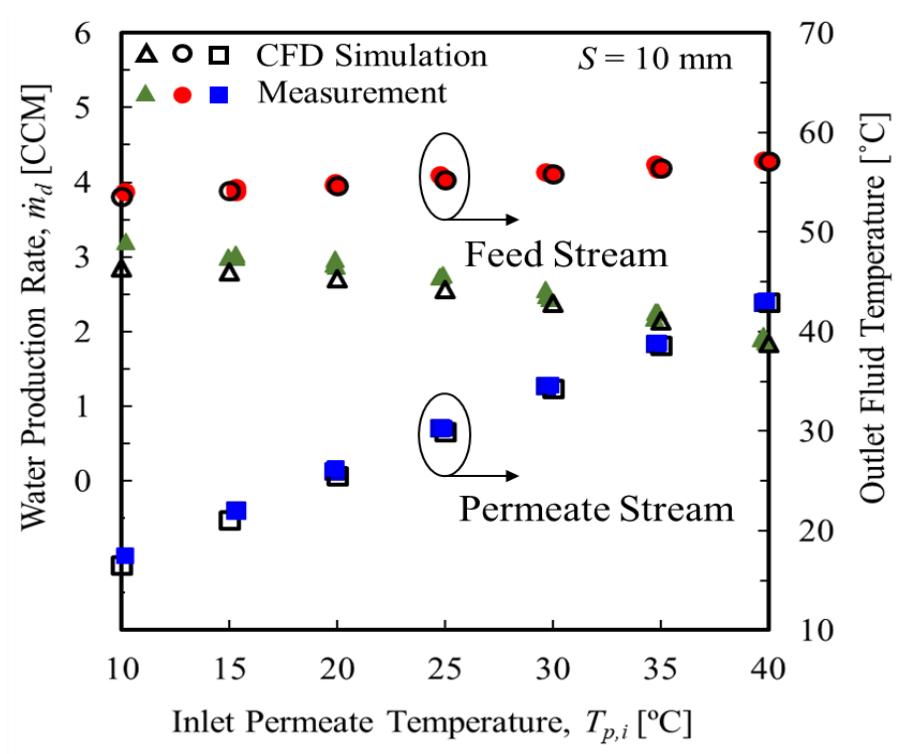

(b)

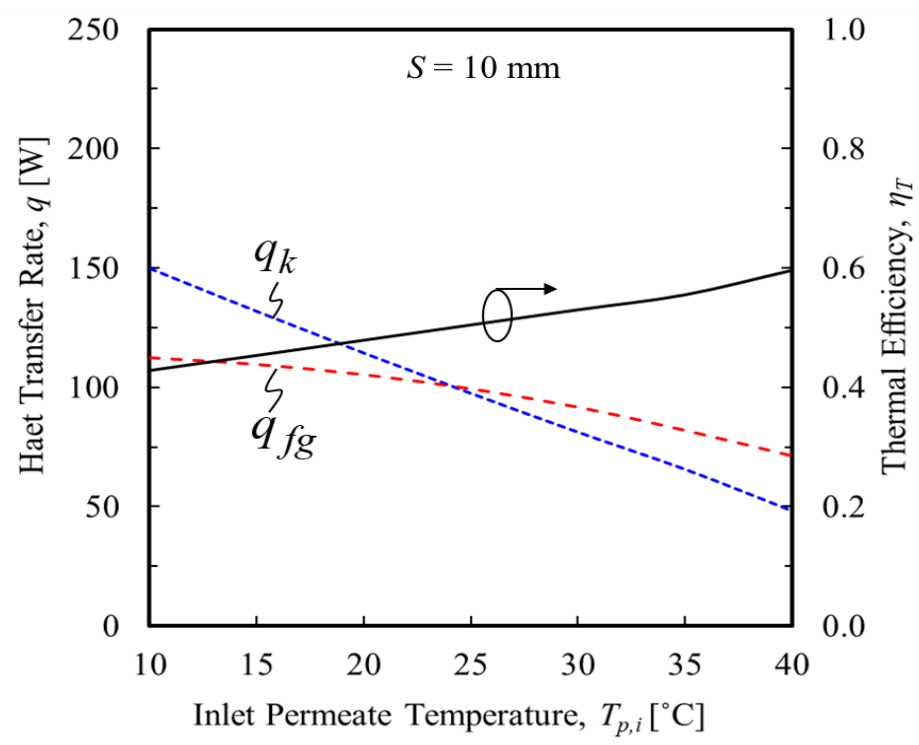

Figure 3.5 Effect of permeate temperature on (a) water production rate and outlet fluid temperature and (b) conduction and phase change heat transfer rates and thermal efficiency of the DCMD system

The effects of the flow rates of the feed and permeate streams on water production were investigated and the results are shown in Fig. 3.6. The flow rates for the feed and permeate loops are equivalent in this study. The baseline flow rate is $9.7 \mathrm{~g} / \mathrm{s}(581 \mathrm{CCM})$ 
which corresponds to an inlet velocity of $6.3 \mathrm{~cm} / \mathrm{s}\left(U_{f, i}=U_{p, i}\right)$. The lower bound of the inlet velocity is $3.1 \mathrm{~cm} / \mathrm{s}(4.8 \mathrm{~g} / \mathrm{s}$ or $290 \mathrm{CCM}$ at room temperature $)$ and the upper bound is $9.4 \mathrm{~cm} / \mathrm{s}(14.5 \mathrm{~g} / \mathrm{s}$ or $872 \mathrm{CCM})$. Figure $3.6(\mathrm{a})$ shows that as the flow rates for the feed and permeate flows are increased, the water production increases because higher flow rates create more uniform temperatures in the feed and permeate channels. Figure 3.6(b) shows that the conduction and phase change heat transfer increase with the flow rate due to the increase in temperature difference between feed and permeate streams. The thermal efficiency also increases as shown in Fig. 3.6(a). The maximum error between the experimental and numerical results for the water production is $15 \%$ at the lower bound flow rate $(290 \mathrm{CCM})$ where the effect of the heat loss to ambient is relatively large compared to the heat transfer in the MD system. 
(a)

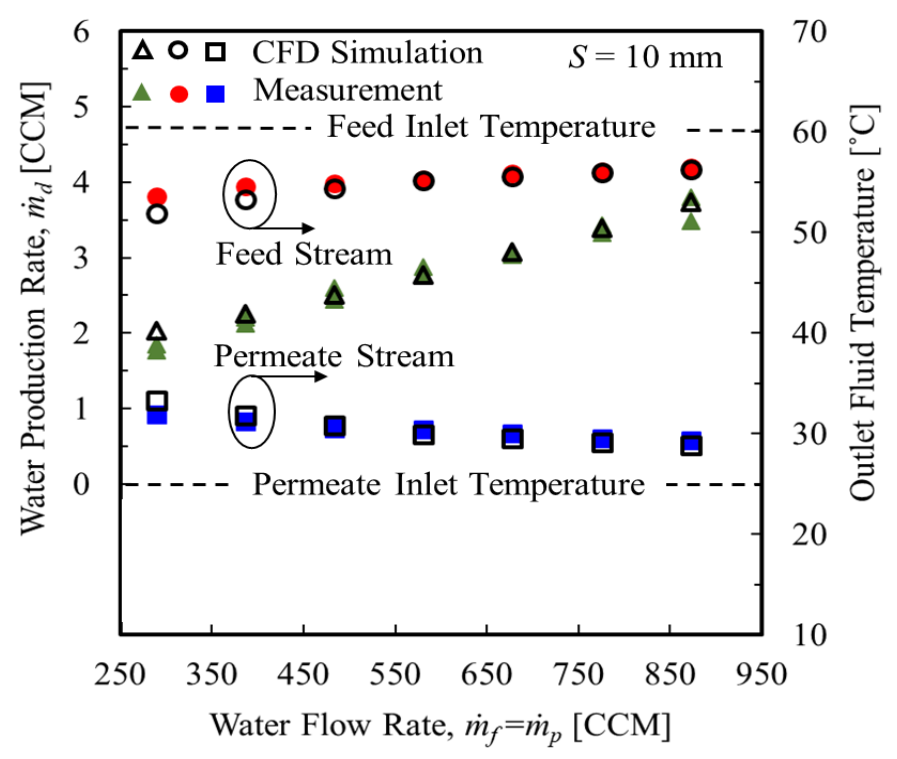

(b)

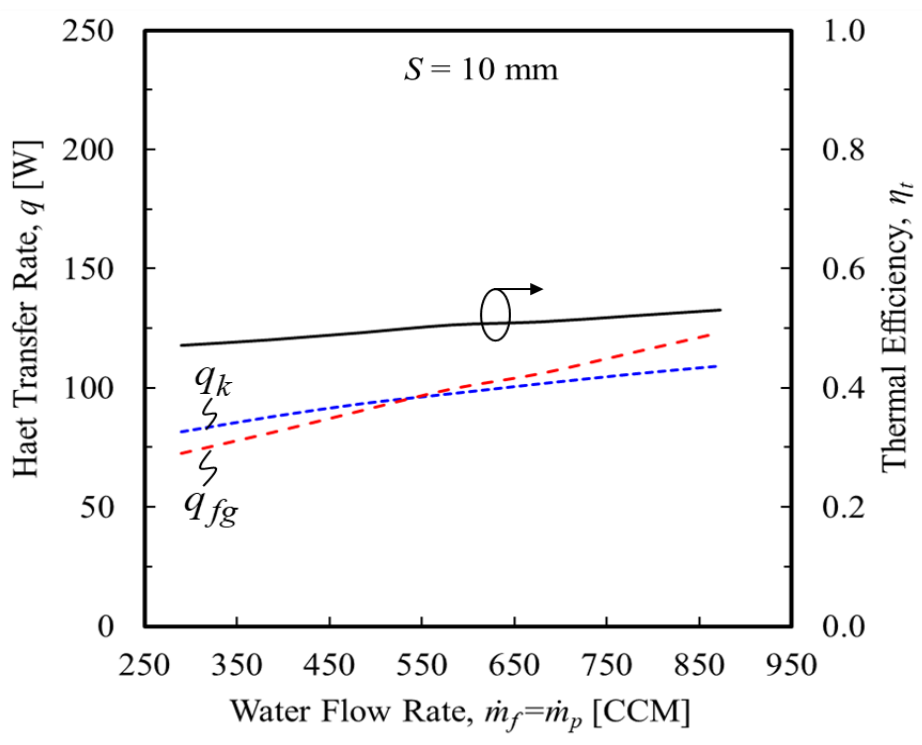

Figure 3.6 Effect of mass flow rates of the feed and permeate channels on (a) water production rate and outlet fluid temperature and (b) heat transfer rates and thermal efficiency of a DCMD system

Figure 3.7 shows the effect of the salinity of $\mathrm{NaCl} /$ water mixtures on the water production performance. The concentrations of the salt water experiments range from 0 (pure water) to $0.0457 \mathrm{~kg}-\mathrm{NaCl} / \mathrm{kg}$ (0.014 mole fraction). This range captures the average 
salinity of seawater, which is about $0.035 \mathrm{~g}-\mathrm{NaCl} / \mathrm{L}$ ( 0.01 mole fraction). Pure water was used as the baseline condition for the parametric study. The presence of $\mathrm{NaCl}$ in saline water impedes the phase change and reduces the water vapor pressure [Eq. (3.18)]. It is observed in Fig. 3.7(a) that when the salt concentration in the feed water increases, the water vapor pressure and thus, the water production rate decreases. The reduced water production consumes less heat from the feed stream and therefore, the outlet feed temperature increases. By the same reasoning, the permeate stream comes out of the MD module at a lower temperature.

Figure 3.7(b) shows the distribution of the salt concentration on the membrane surface in the feed channel and its associated vapor pressure in the case of the highest salt concentration $\left(C_{f, i}=0.0457 \mathrm{~kg}-\mathrm{NaCl} / \mathrm{kg}\right)$. The salt concentration oscillation is synchronized with the vapor pressure which affects the water production and the salt concentration. The oscillations of the salt concentration and water vapor pressure result from the convergingdiverging flow around the spacer filaments (Fig. 3.3). The peaks of the oscillation coincide with the location of the mesh screen filament where the flow is being choked by the mesh filaments where the flow accelerates. The salt concentration at the outlet is higher than that of the inlet because of the water distillation. The vapor pressure decreases with both higher salt concentration and decreasing feed temperature [Fig. 3.3(c)].

Figure 3.7(c) shows the salt concentration contour in the feed channel for the case of the highest salt concentration. It is observed that a thin concentration boundary layer with higher concentration is formed near the membrane where the water distillation (loss of pure water) occurs. The largest error between experimental and CFD simulation results of the water production rate occurs for the upper bound salinity solution, at 15\%. 
(a)

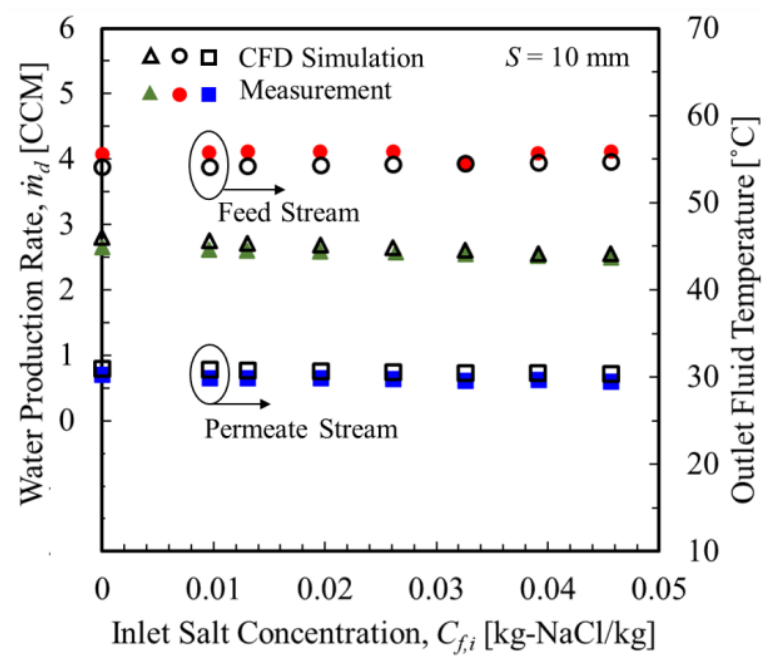

(b)

(c)

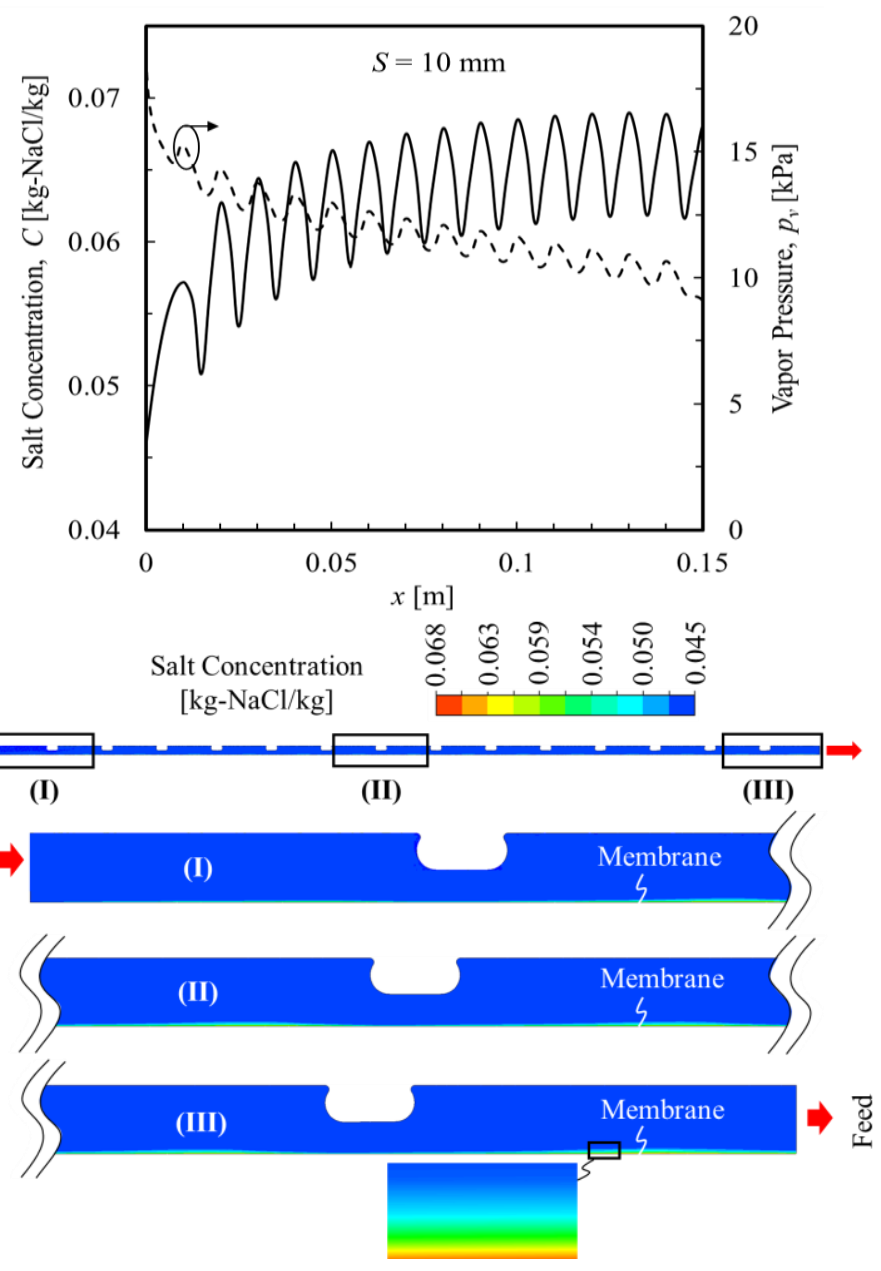

Figure 3.7 (a) Effect of $\mathrm{NaCl}$ concentration in the feed channel on water production rate. (b) Variation of $\mathrm{NaCl}$ concentration on the membrane surface and saturation pressure in the feed channel and (c) contour of the $\mathrm{NaCl}$ concentration in the feed channel for a salt concentration of $C_{f, i}=0.0457 \mathrm{~kg}-\mathrm{NaCl} / \mathrm{kg}$ 


\subsubsection{Effect of Mesh Screen Filament Spacing}

The mesh screen installed in the flow channels [Fig. 3.1(b)] is often used as a spacer to support the flexible membrane in the flow channels. At the same time, it acts as an insert that enhances the convective heat transfer of the channels, which causes a converging (accelerating) and diverging (decelerating) flow through a winding flow path around the mesh screen filaments and also creates a flow mixing and disturbs of the boundary layers formed on the membrane surfaces [Figs. 3.3(a-b)]. Recall that the filament spacing of the mesh spacer used for this study as the baseline condition is $10 \mathrm{~mm}$. To study the effect of the mesh spacer on the water production performance, two additional cases - (i) no spacer and (ii) a mesh spacer with a filament spacing $5 \mathrm{~mm}$ - were analyzed.

Figures 3.8(a) and (b) show various thermal resistances and heat transfers taking place in the membrane module for the $5 \mathrm{~mm}$ and $10 \mathrm{~mm}$ screen filament spacing and the case with no mesh screen spacer. In Fig. 3.8(a), four different thermal resistances, defined per surface area [Eqs. (3.22-3.26)], were compared. The total thermal resistance $\left(R_{T}^{\prime \prime}\right)$ is composed of the convective thermal resistances $\left(R_{f}^{\prime \prime}\right.$ and $\left.R_{p}^{\prime \prime}\right)$ in the feed and permeate channels and a parallel combination $\left(R_{k, f g}^{\prime \prime}\right)$ of the conduction and phase change thermal resistances (Fig. 3.2). As shown in Fig. 3.8(a), the convective thermal resistances decrease with the insertion of mesh screen spacers, especially one with smaller spacing between mesh screen filaments. This is due to the aforementioned converging-and-diverging flow in the feed and permeate channels.

Figure 3.8(b) shows the membrane surface temperatures in the feed and permeate channels. It is observed that as the mesh filament spacing is decreased, the difference in 
the membrane surface temperatures and therefore, the conduction and phase change heat transfers are increased, leading to higher vapor pressure differences between the feed and permeate streams and subsequent transmembrane mass flux enhancement [Fig. 3.8(c)].

The enhancement of the convection heat transfer (a reduction in the convective thermal resistances) in the flow channels is mainly attributed to the increased area affected by the accelerating flow (converging) in the narrowed region below the mesh spacer filaments. The reduced convection thermal resistances are also manifested by increased Temperature Polarization Coefficients [TPC, Eq. (3.28)] as shown in Fig. 3.9, which makes the membrane surface temperature in each channel approach its bulk fluid temperature. Figure 3.9 also shows the Concentration Polarization Coefficient [CPC, Eq. (3.29)] of the highest salt concentration case $\left(C_{f, i}=0.0457 \mathrm{~kg}-\mathrm{NaCl} / \mathrm{kg}\right)$ [see Figs. 3.7(b,c)]. A higher CPC means a smaller difference between the bulk fluid concentration to the concentration on the membrane surface. The changes in the CPC follows the trend found in the TPC. Such a coupling between momentum, heat and mass transfer are expressed by the ChiltonColburn $J$-factor analogy [32]. The smaller the screen spacing gets, the less the polarization effects are. 
(a)

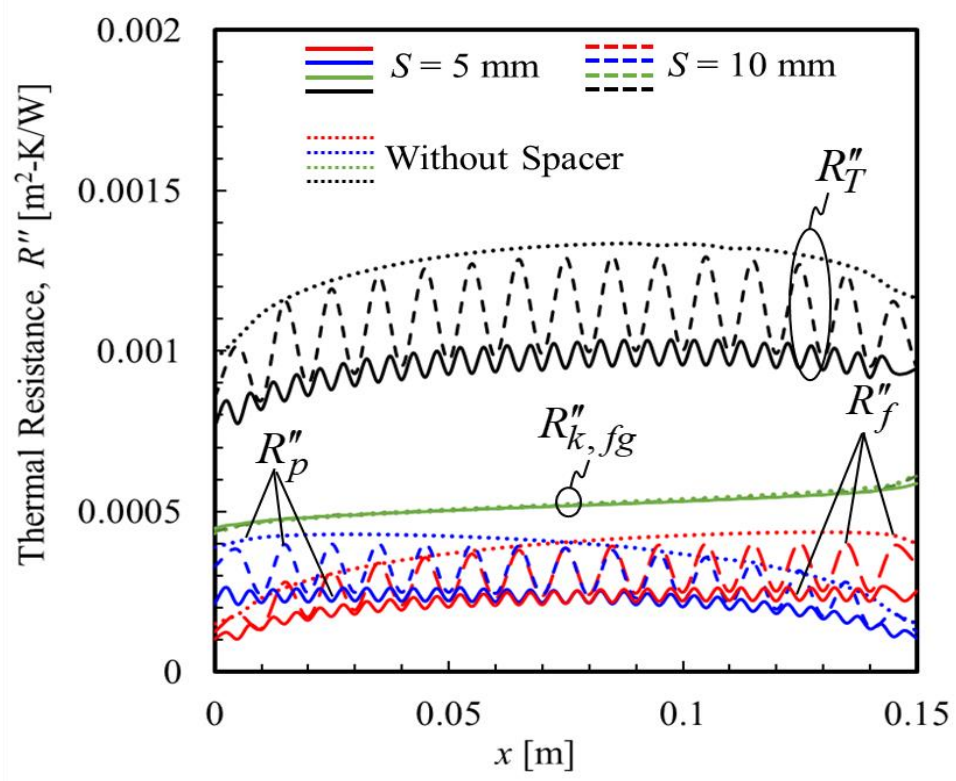

(b)

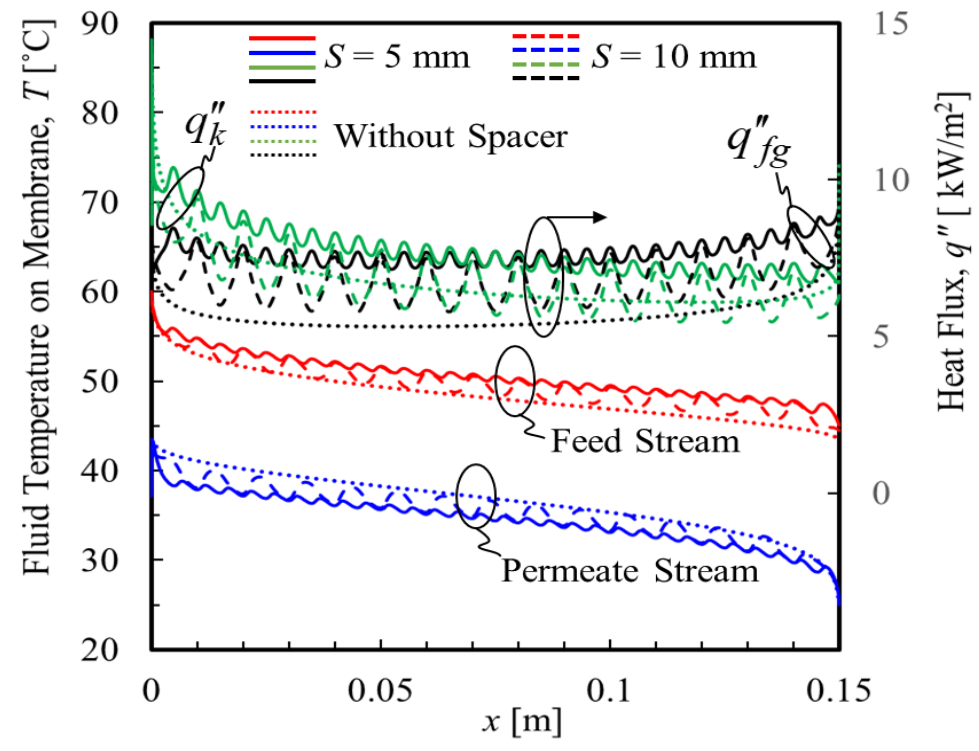


(c)

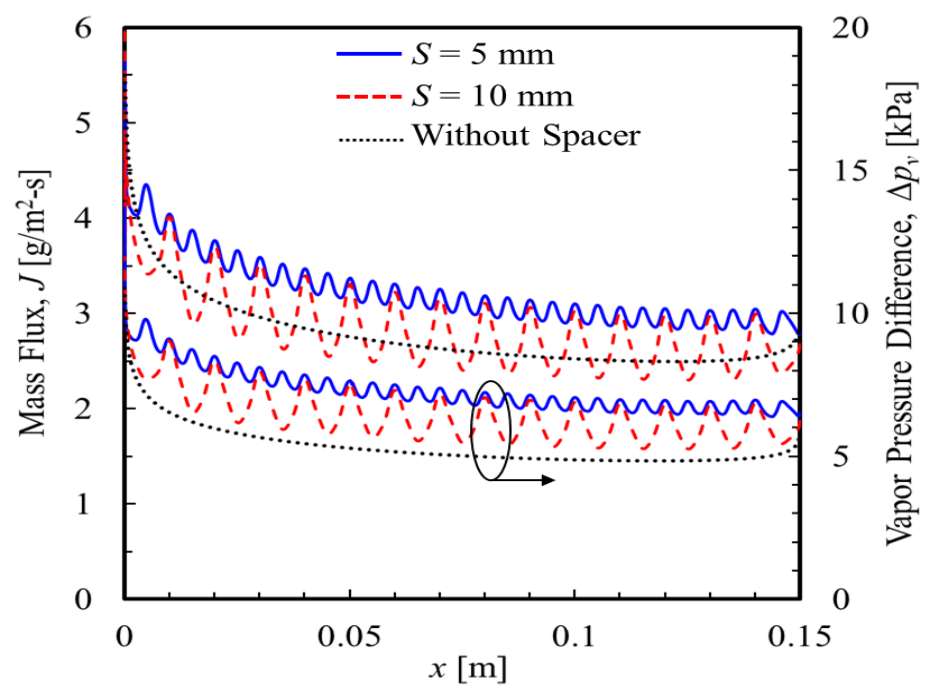

Figure 3.8 Effect of non-spacer and spacer-filled channels with mesh screen filament spacings of $10 \mathrm{~mm}$ and $5 \mathrm{~mm}$ on (a) thermal resistances including convection thermal resistance in the feed (red lines) and permeate (blue lines) streams, combination of conduction and phase change thermal resistances (green lines) and total thermal resistance (black lines) (b) membrane surface temperatures in the feed (red lines) and permeate (blue lines) channels and conduction (green lines) and phase change (black lines) heat fluxes and (c) mass fluxes and differences of saturation pressure between the feed and permeate channels

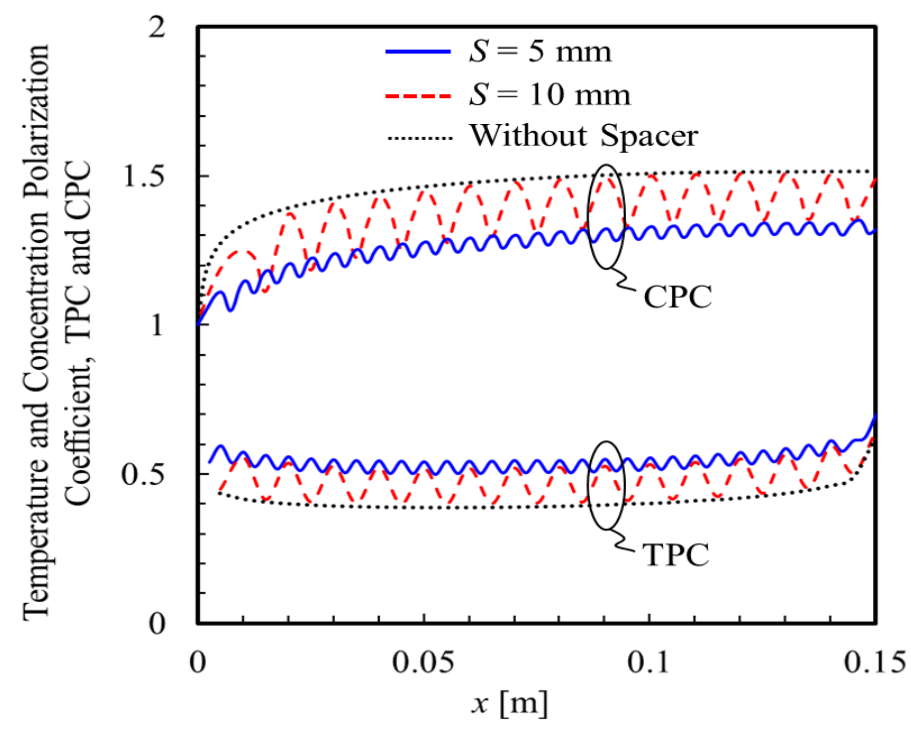

Figure 3.9 Variations of temperature and concentration polarization coefficients for non-spacer and spacer-filled channels with mesh screen filament spacings of $10 \mathrm{~mm}$ and $5 \mathrm{~mm}$. The concentration polarization coefficients are based on a salt concentration of $C_{f, i}=0.0457 \mathrm{~kg}-\mathrm{NaCl} / \mathrm{kg}$ 


\subsubsection{Effect of Flow Configuration}

Two possible flow configurations for DCMD systems are counter and parallel flows. A counterflow configuration is the baseline condition for the results in the previous graphs. A parallel flow configuration was considered for the numerical analysis and the results are shown in Fig. 3.11. In the parallel configuration, the permeate stream flows from left to right (i.e., $x$-direction), identical to the feed stream flow.

Figure 3.11(a) shows the comparison between the thermal resistances for the two flow configurations. The convection thermal resistance is the lowest at the inlets of each flow configuration because of the entrance effect of a developing flow [32]. As the flow continues to pass through the channels, the flow gets more developed, and the averaged convection thermal resistances increase and converge to the values of a fully-developed flow. Note that the left side of Fig. 3.11(a) is the inlet of the feed stream and the right side is the inlet of the permeate stream in a counterflow configuration, while the left side is the inlets of both the feed and permeate streams for a parallel flow configuration. The mixed inlet/outlet configuration of the counterflow results in a more uniform total thermal resistance.

Figure 3.11(b) shows that the temperature profiles of the parallel feed and permeate streams quickly converge into each other along the flow direction and thus, the temperature difference and associated heat transfer between the feed and permeate streams decrease exponentially. Recall that the temperature difference in the counterflow configuration is approximately uniform [Fig. 3.4(c)]. Figure 3.11(c) shows the profiles of the saturation pressure, pressure difference, and transmembrane mass flux of the parallel configuration. These results rapidly decrease because of the exponential decrease in the temperature 
difference shown in Fig. 3.11(b). In the parallel configuration, the inlet temperatures of the feed and permeate flows are highest and lowest, respectively. The increased inlet temperature difference with the lowest inlet temperature of the permeate stream excessively increases the pressure difference at the inlet but this effect is limited to a short distance from the inlet. Overall, the water production in the parallel flow configuration is worse than the counterflow configuration. In the baseline conditions, the water production of the parallel flow is $4 \%$ less than that of the counterflow.

(a)

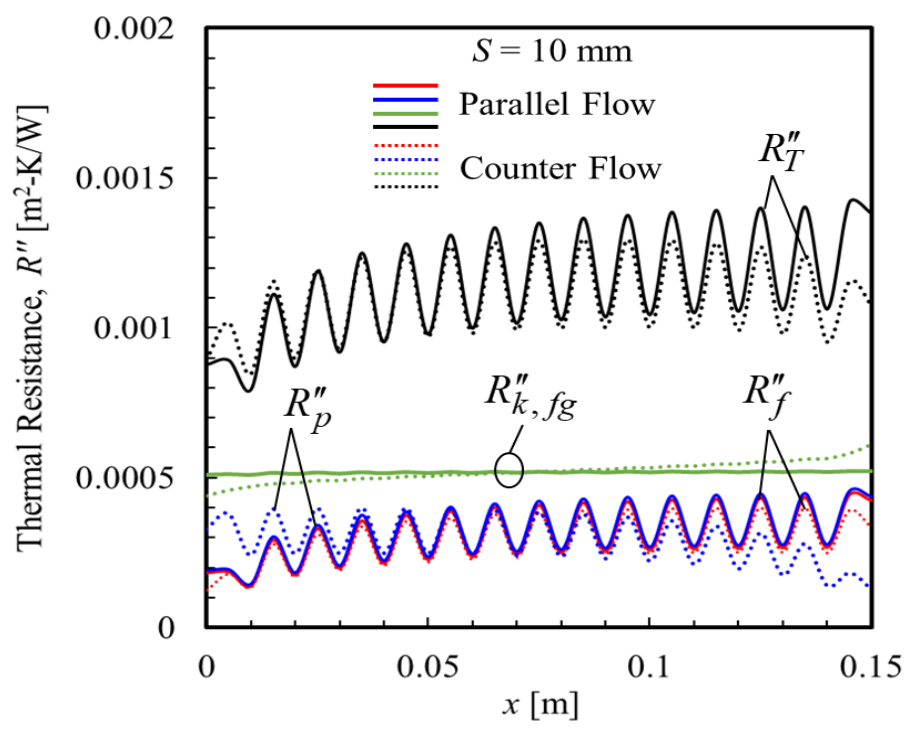




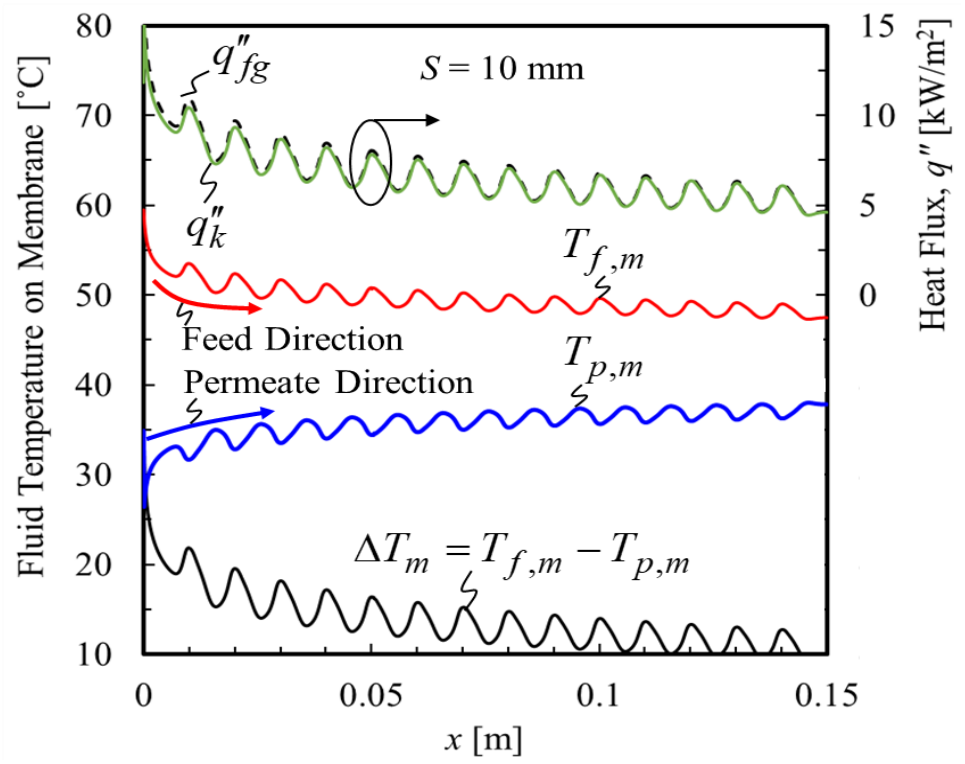

(c)

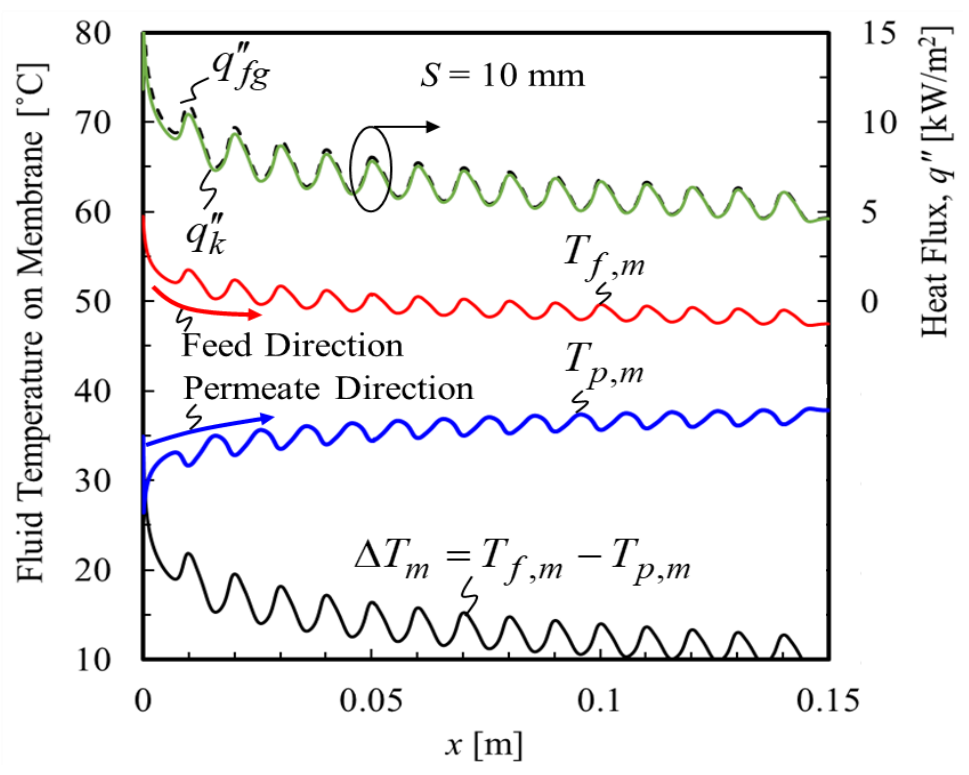

Figure 3.10 Variations of (a) thermal resistances for counter and parallel flows including convection thermal resistance in the feed (red lines) and permeate (blue lines) streams, combination of conduction and phase change thermal resistances (green lines) and total thermal resistance (black lines) (b) membrane surface temperatures in the feed (red solid line) and permeate (blue solid line) channels, temperature difference between feed and permeate streams (black solid line) and conduction (green solid line) and phase change (black dashed line) heat fluxes and (c) vapor pressures (red and blue solid lines), differences of vapor pressure between the feed and permeate channels (black dashed line) and mass flux of the membrane module (black solid line) for parallel flow arrangement and mesh screen filament spacing of $10 \mathrm{~mm}$ 


\subsection{SUMMARY}

Computational Fluid Dynamics (CFD) model of a direct contact membrane distillation (DCMD) considering transmembrane mass flux and heat conduction and associated phase change heat transfer (evaporation and condensation) was developed. The CFD model analyzed the effects of filament spacing of a screen spacer in the flow channels and flow configuration (counterflow and parallel) on the water distillation performance. This study concluded that high water distillation is achieved by using low permeate temperature, high feed temperature, high feed and permeate flow rates, and less salt concentration in a counterflow configuration. The water distillation is more influenced by the feed temperature than the permeate temperature due to the water saturation pressuretemperature relation. The simulation results also showed that the convection thermal resistances in the feed and permeate channels are decreased by using screen spacers, especially one with smaller spacing between mesh screen filaments, and therefore, the temperature and concentration polarization effects are decreased in the feed and permeate channels. 


\section{CHAPTER 4: SCALING EFFECT ON PERFORMANCE OF MEMBRANE DISTILLATION CRYSTALLIZATION FOR HYPERSALINE BRINES}

The global water crisis for drinking, industrial, and irrigation purposes has become a serious threat for populations all around the world. The most promising solution for the water issue is the desalination of seawater or brackish water. The main commercially available desalination technologies are categorized into thermal and membrane processes. Due to the energy-intensive processes in thermal technologies, membrane technologies have progressed remarkably in the past few decades [33].

Among membrane technologies, reverse osmosis (RO) is one of the common membrane technologies to produce drinking water. However, a huge volume of brine with high salinity is generated from $\mathrm{RO}$ technology $[34,35]$. The $\mathrm{RO}$ bine is dumped into rivers and the treatment of the RO brine is critical to reduce the harmful effect on the ecosystem. Membrane distillation (MD) is an appropriate membrane purification technology to treat the RO brine since this technology has illustrated a high water rejection performance for highly concentrated brine [36]. After water recovery by MD technology, the output brine can be processed by a crystallization equipment which recovers inorganic salt from the brine [37]. The combination of MD and crystallization processes is called membrane distillation crystallization technology to convert the RO brine into purified water and salt crystal. Therefore, by membrane distillation crystallization process, the output brine of the RO system is treated in order to reduce negative environmental impact.

Membrane distillation is a hydrophobic membrane utilizing the distillation process at a lower operating temperature than boiling temperature to produce purified water. There 
are four different configurations for MD including direct contact, air gap, sweeping gas, and vacuum membrane distillation [38]. In direct contact membrane distillation (DCMD), a common configuration, there are two streams with different temperatures called feed and permeate streams and a membrane in between. The vapor pressure difference between feed and permeate streams is the driving force to transport water vapor through the membrane. Due to the fouling resistance [39], less sensitivity to feed concentration [40] and a small volume of discharge, the MD has been considered as one of the most efficient technologies for recovery of highly concentrated brine [41]. The MD can be coupled with other systems when waste heat, solar [42] or geothermal [9] sources are available. The high reliability of MD systems at a highly concentrated feed water solution provides the possibility of running the MD system up to saturation condition in the feed water and treating the brine of the MD in a crystallizer to produce salt crystals. The membrane distillation crystallization (MDC) [43-45] can be part of a zero liquid discharge system [46] as a target to eliminate all the liquid from the RO brine which is harmful to the ecosystem if it is dumped into waterways without treatment.

In this study, a membrane distillation crystallization was used to recover purified water from highly concentrated $\mathrm{NaCl}$ solutions and produce $\mathrm{NaCl}$ crystals. The effects of Sodium Chloride concentration from zero to saturation condition, inlet feed and permeate temperatures and flow rate of feed and permeate on the MD performance were studied. For the crystallization part, the effect of the cooling rate was investigated on the crystal size, and the generated crystals were analyzed under a microscope to determine their sizes. 


\subsection{EXPERIMENTAL METHOD}

Figure 4.1 illustrates the schematic of the experimental set-up for DCMD. The DCMD system includes feed water and permeate water loops. Each loop has a fluid reservoir made of CPVC piping, a DC pump, a heater (feed water loop) or a chiller (permeate water loop), and a rotameter flow meter. To measure inlet and outlet feed and permeate temperature of MD modules, four thermocouples were used. The membrane is made of PTFE membrane by CLARCOR's manufacturer with a model number of QM022 and size of $150 \mathrm{~mm}$ in length and $95.3 \mathrm{~mm}$ in width. Two polypropylene mesh screens and acrylic plates support the membrane at the top and bottom. The detailed design for the MD experiment set-up was explained in our previous work. A new crystallization loop was added to the existing MD set-up to produce salt crystal from the saturated feed water generated by the MD operation. This crystallization loop consisted of a crystallization reservoir, a chiller, and a filtration set-up. Salt crystals were filtered with a $2 \mu \mathrm{m}$ cellulose paper from Whatman. 


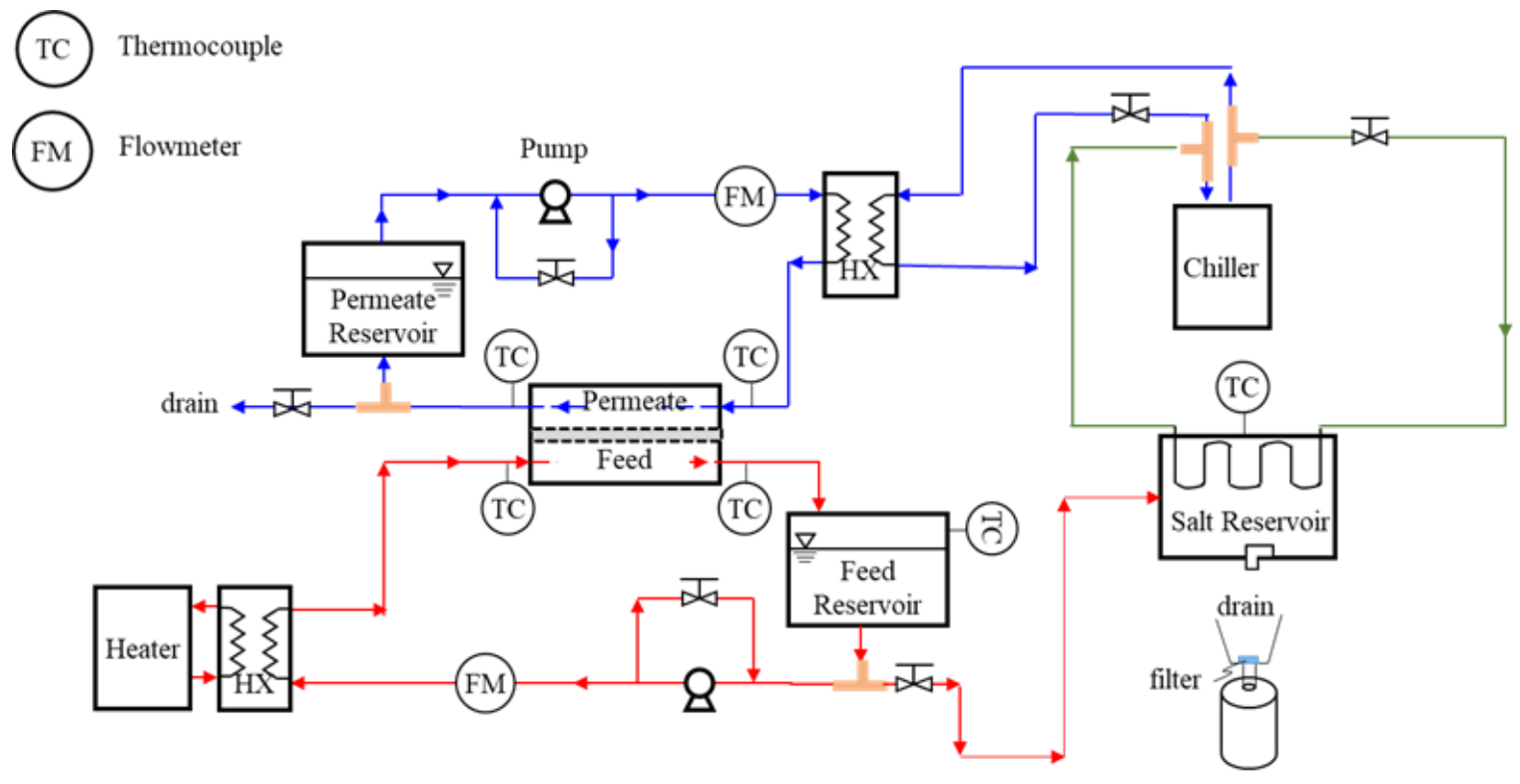

\section{Figure 4.1 Schematic of the MC experimental setup}

The steady-state condition is captured when the readings of temperature and flow rate stay in the range of desired temperature $\pm 0.5^{\circ} \mathrm{C}$ and flow rate $\pm 2 \mathrm{CCM}$. An average water production is determined after 60 minutes running in the steady-state condition. To ensure repeatability of the experiment, each case was tested three times. The scale measuring water production has an error of $\pm 1 \mathrm{~g}$. The temperature measurement using T-type thermocouple has an error of $\pm 1^{\circ} \mathrm{C}$.

In the feed water loop, salty water from small concentrations of $\mathrm{NaCl}$ (Fisher Chemical, S271-1) was circulated to a near saturation condition to find the effect of salt concentration on the distilled water output. The concentration of $\mathrm{NaCl}$ was measured by a digital refractometer (Hanna Instruments, HI96811). In addition, the density measurement was used to assess the salinity of a sample solution. The feed water volume in pluming was calculated by calibration curve, feed water reservoir volume and $\mathrm{NaCl}$ mass. Therefore, an 
accurate amount of $\mathrm{NaCl}$ was used to add the total volume to prepare the desired concentration for the experiment.

After running the MD operation up to the saturation condition measured by the amount of water production, the feed water was transferred to the crystallization reservoir for cooling. The temperature inside the crystallization reservoir was measured by a T-type thermocouple to reach the ambient temperature by forced or natural cooling methods. Then, the generated $\mathrm{NaCl}$ crystals were filtered to collect for analyzing under the microscope (Eclipse LV100, Nikon) to determine the size distribution.

\subsection{NUMERICAL AND ANALYTICAL ANALYSIS}

The distilled water flux of the membrane distillation depends on the salinity of feed water. Regarding the feed water salinity, the water flux can be split into two regimes. In the first regime, there is a gradual decrease in the distilled water flux due to the effect of salinity on the depression of vapor pressure. In the second regime, there is a sharp drop in the distilled water flux due to the blockage of open surface by the deposition of $\mathrm{NaCl}$ crystals on the membrane surface. The first regime was simulated by a computational fluid dynamics (CFD) model and second regime was captured by an analytical approach with adjusting parameters from the MD experiment. A CFD model was performed by ANSYS FLUENT to capture the heat and mass transfer in the DCMD. The CFD model was explained in chapter 3 section 3.1. The water vapor flux is expressed by

$$
J=\frac{\left(\varepsilon_{m} / \tau_{m}\right) p_{T} D_{w v-a} M_{w}}{(1-\alpha) \delta_{m} R T_{m}} \ln \left\{\frac{D_{K n}\left[p_{T}-(1-\alpha) p_{v, p}\right]+\left(\varepsilon_{m} / \tau_{m}\right) p_{T} D_{w v-a}}{D_{K n}\left[p_{T}-(1-\alpha) p_{v, f}\right]+\left(\varepsilon_{m} / \tau_{m}\right) p_{T} D_{w v-a}}\right\},
$$




$$
\begin{aligned}
& D_{K n}=\frac{4 \varepsilon_{m}}{3 \tau_{m}} d_{p} \sqrt{\frac{R T_{m}}{2 \pi M_{w}}}, \\
& p_{T} D_{w v-a}=1.895 \times 10^{-5} T_{m}^{2.072}, \\
& \alpha=\frac{M_{w}}{M_{a}}
\end{aligned}
$$

where $\tau_{m}, \varepsilon_{m}, \delta_{m}$, and $d_{p}$ are the properties of the membrane including tortuosity, porosity, thickness, and pore diameter of the membrane, respectively, and their values are tabulated in Table 4.1. $T_{m}$ is the average temperature of the membrane at the interface of membrane and channels $\left(T_{f, m}, T_{p, m}\right) . M_{w}$ and $M_{a}$ represent the molecular weight of water and air, respectively. In the second regime, when there was a sharp decrease in the water flux due to the blockage of open surface in the membrane, an analytical approach was used. The reduction in the open surface is proportional to the feed flowrate intensifying the tangential force in the force balance. In addition, the rate of pore blockage relates to the current pore condition. Therefore, the rate of blockage is formulated as

$$
\begin{aligned}
& \frac{d a}{d t} \propto a Q_{f}, \\
& a=\varepsilon_{m} A_{m},
\end{aligned}
$$

where $Q_{f}$ is the volumetric flow rate $\left[\mathrm{m}^{3} / \mathrm{s}\right]$ in the feed channel and $a$ is the open surface of the membrane which is defined by the product of porosity and membrane surface.

A variable representing the ratio of the blocked area to the permeate volume was defined by Hermia [47] to correlate the rate of the decrease in the open surface to the feed water flow rate. This correlation was modified by Ramezanianpour et. al. [48] and they 
introduce a new variable as the ratio of roughness to the hydraulic diameter of the channel $\left(C_{m} e / d_{h}\right)$ to capture the effect of roughness of the deposited salt crystals on the membrane. The blocked area to permeate volume $(\xi)$ is defined below and it was used to correlate the rate of the blockage area to the feed water flow rate

$$
\begin{aligned}
& \xi=\frac{3 \rho_{f} m_{p}}{2 \rho_{p} d_{s} \psi} \frac{C_{m}}{d_{h}} e, \\
& \frac{\mathrm{d} a}{\mathrm{~d} t}=-\xi \frac{a}{a_{0}} Q_{f},
\end{aligned}
$$

where $\rho_{f}$ and $\rho_{p}$ are the density of feed water and $\mathrm{NaCl}$ salt, respectively. $d_{s}$ is the mean particle size of salt crystals, $\psi$ is the shape factor, $m_{p}$ is the mass fraction of $\mathrm{NaCl}$ in the water, $C_{m}$ is the coefficient of deposited mass relating the effect of roughness on residual mass, $e$ is the effective roughness height, and $d_{h}$ is the hydrodynamic diameter of the feed channel.

To adhere a particle to the membrane surface, the force balance in Fig. 4.2 results in

$$
\begin{aligned}
& F_{T}=\eta\left(F_{A}-F_{L}\right), \\
& F_{T}=C_{T} d_{s} e, \\
& C_{T}=\frac{24 \pi \mu Q_{f}}{A_{m} d_{h}}, \\
& F_{A}=C_{A} d_{s}, \\
& F_{L}=-C_{L} d_{s} \frac{\mathrm{d} e}{\mathrm{~d} t},
\end{aligned}
$$




$$
\begin{aligned}
& C_{L}=3 \pi \mu \varphi, \\
& \varphi=\sqrt{\frac{1}{3} \frac{d_{s}}{\delta_{m}}+1.149}
\end{aligned}
$$

where $F_{T}$ is the tangential force exerting on the crystals from the fluid. $F_{A}$ is the adhesion force between sodium chloride crystal and membrane surface and $F_{L}$ is the lubrication force resisting motion of crystals due to the roughness of the membrane. $C_{T}$ is the stress coefficient of tangential force and it depends on the volumetric flow rate $\left(Q_{f}\right)$, hydraulic diameter $\left(d_{h}\right)$ and membrane surface $\left(A_{m}\right) . C_{A}$ is the adjusting parameter which depends on the membrane material, type of salinity, operating condition in the experiment and is determined from the experimental data. $C_{L}$ is a lubrication constant depending on viscosity $(\mu)$ and permeable surface factor $(\varphi)$ and is defined by Goren [49].

By substituting Eqs. (4.10-4.15) into Eq. (4.9) and integrating with respect to time, it is seen that the equivalent roughness $(e)$ depends on time $(t)$ as follows

$$
\frac{\eta \ln \left(C_{T} e-\eta C_{A}\right)}{C_{T}}=\frac{t}{C_{L}}+C_{1},
$$

where the integration constant of $C_{1}$ is determined by the original roughness of the polypropylene membrane surface which is $15 \mathrm{~nm}$ at $t=0$. The equivalent roughness is calculated as given

$$
e=\frac{\exp \left[\frac{C_{T}}{\eta}\left(\frac{t}{C_{L}}+C_{1}\right)\right]+\eta C_{A}}{C_{T}} .
$$

Substituting Eq. (4.17) into Eq. (4.16) and integrating with respect to time, the result is given as 


$$
a=\frac{J_{0}}{a_{0}} \exp \left\{-\frac{3 \rho_{f} m_{p} C_{m} Q_{f}}{2 \rho_{p} d_{s} \psi d_{h} C_{T}^{2} a_{0}}\left[C_{L} \exp \frac{C_{T}}{\eta}\left(C_{1}+\frac{t}{C_{L}}\right)+\eta C_{T} C_{A} t\right]\right\}-C_{2},
$$

where the constant of integration $C_{2}$ is determined regarding the initial open area $\left(a_{0}\right)$. The values of the constant variables in Eq. (4.18) are listed in Table 4.2.

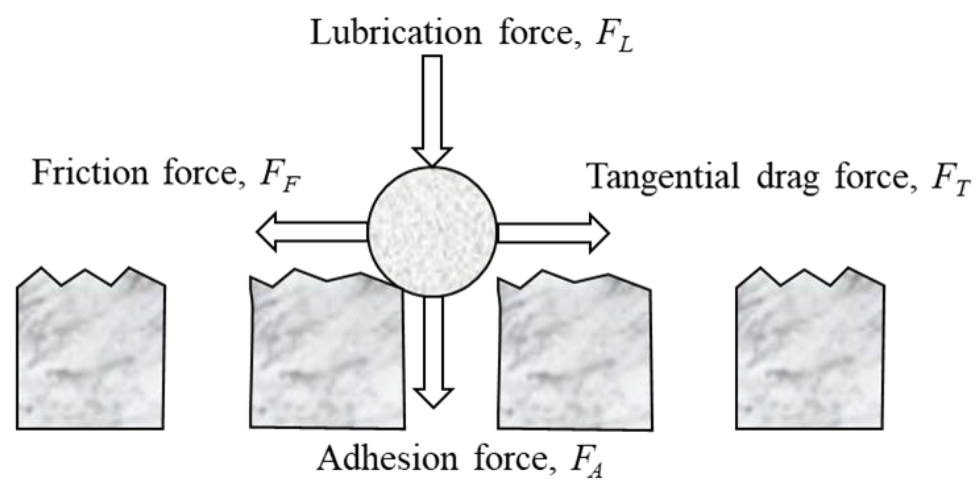

Figure 4.2 Force balance on a $\mathrm{NaCl}$ crystal

Table 4.1 Dimensional and thermophysical properties of the membrane used in the DCMD experiment

\begin{tabular}{ccccccc}
\hline $\begin{array}{c}\text { Membrane } \\
\text { type }\end{array}$ & $\delta_{m}$ & $d_{p}$ & $\varepsilon_{m}$ & $\tau_{m}$ & $k_{m}$ & $\theta_{c}$ \\
& {$[\mathrm{~m}]$} & {$[\mathrm{m}]$} & {$[-]$} & {$[-]$} & {$[\mathrm{W} / \mathrm{m}-\mathrm{K}]$} & $\left.{ }^{\circ}\right]$ \\
\hline QM022 & $84 \times 10^{-6}$ & $0.36 \times 10^{-6}$ & 0.62 & 2.34 & 0.23 & 127 \\
\hline
\end{tabular}

Table 4.2 Constant parameters used in Eq. (4.18)

\begin{tabular}{ccc}
\hline$C_{m}$ & $\psi$ & $d_{s}$ \\
{$[-]$} & {$[-]$} & {$[\mathrm{m}]$} \\
\hline 0.08 & 0.6 & $5 \times 10^{-5}$
\end{tabular}




\subsection{RESULTS AND DISCUSSION}

The membrane distillation crystallization experiments were performed to investigate the effects of the $\mathrm{NaCl}$ concentration, inlet feed and permeate temperatures and flow rates on the MD performance. The operating conditions in the experiment are listed in Table 4.2. After running the MD operation, the brines of the MD for three different inlet feed temperatures $\left(45^{\circ} \mathrm{C}, 60^{\circ} \mathrm{C}\right.$, and $\left.75^{\circ} \mathrm{C}\right)$ were treated by the crystallization unit by two natural and forced cooling processes to produce the $\mathrm{NaCl}$ crystals. The results of $\mathrm{CFD}$ and analytical models were compared with the experimental data to illustrate the validity range of each model.

\subsubsection{Effect of Salt Concentration}

The results of water flux, outlet feed and permeate water temperatures are shown in Fig. 4.3. The $\mathrm{NaCl}$ concentration was varied from zero to a highly concentrated solution to $0.328[\mathrm{~kg}-\mathrm{NaCl} / \mathrm{kg}]$. The water flux decreases with the concentration of the feed water solutions and CFD results of water flux are in good agreement with experimental results. The decrease in the water flux at small and mildly concentration of feed water is due to the suppression of the vapor pressure in the feed water. But, at a highly concentrated feed water solution, around $0.3[\mathrm{~kg}-\mathrm{NaCl} / \mathrm{kg}]$, the water flux of the CFD simulation slightly deviates from the water flux in the experiment and this deviation shows the trace of the scaling effect where the $\mathrm{NaCl}$ crystals are deposited on the membrane surface which results in the blockage of the membrane pores. As the water flux decreases, feed and permeate water leave the channels with a smaller change in the temperature. Therefore, the outlet feed water temperatures are higher, and the outlet permeate water temperatures are lower. 


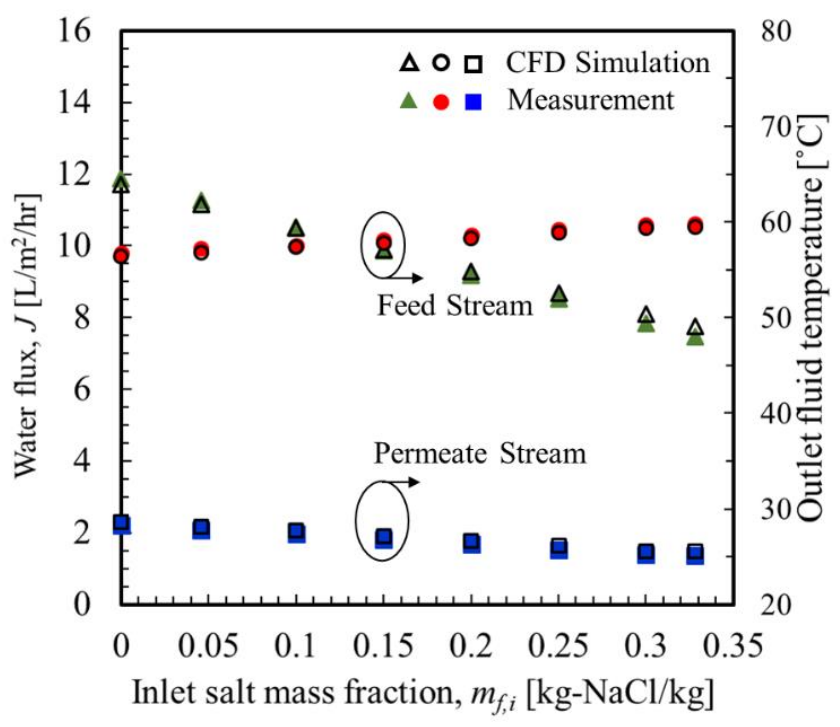

Figure 4.3 Effect of feed water concentration on water flux, outlet feed, and permeate water temperatures

\subsubsection{Effect of Inlet Feed and Permeate Temperatures}

The effects of feed inlet temperature are illustrated in Fig. 4.4 when the feed water concentrate is $0.2[\mathrm{~kg}-\mathrm{NaCl} / \mathrm{kg}]$. The results show that the water flux increases by increasing inlet feed temperature due to the increase in the pressure difference in Eq. (4.1). Although the saturation pressure is increased exponentially with temperature for pure water, the vapor pressure of salt water is suppressed by the smaller water activity. By increasing the inlet feed temperature, the permeate water exits the channel with higher temperatures due to the higher water flux. The effects of inlet permeate temperature on water flux, feed and permeate outlet temperatures are shown in Fig. 4.5. By increasing the inlet permeate temperature, the water flux decreases due to the smaller temperature difference across the membrane and therefore outlet feed temperatures are higher. 


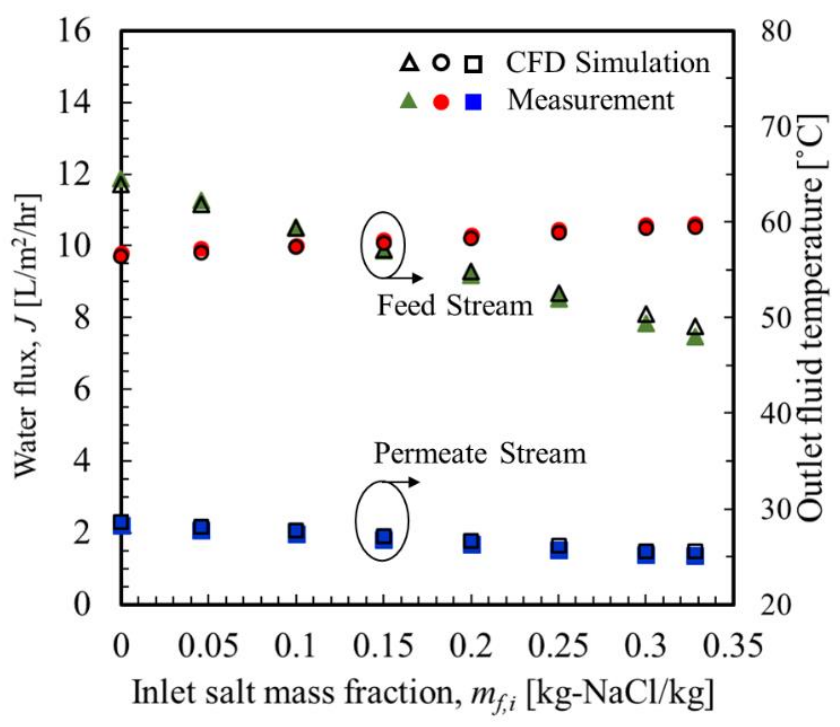

Figure 4.4 Effect of inlet feed temperature on water flux, outlet feed, and permeate temperatures

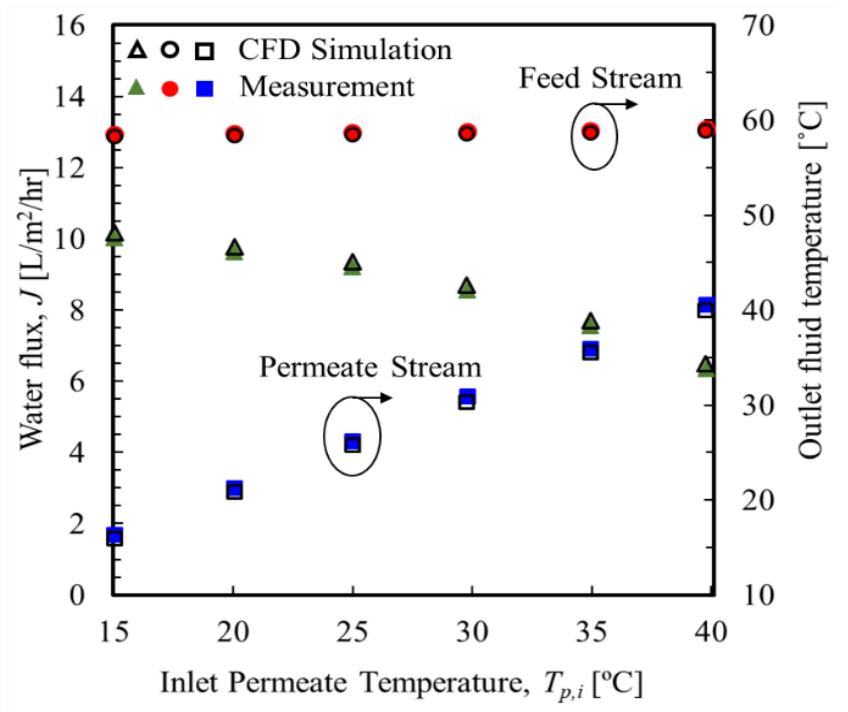

Figure 4.5 Effect of inlet permeate temperature on water flux, outlet feed and permeate temperatures

\subsubsection{Effect of Flow Rate}

The experimental and numerical results of water flux, feed and permeate outlet temperatures for different water flow rates in the feed and permeate channels are shown in 
Fig. 4.6. It is noteworthy that the flow rates in the feed and permeate channels are the same and the flow rates were varied from $290 \mathrm{CCM}$ to $872 \mathrm{CCM}$. The water flux increases by increasing the flow rate in the feed and permeate channels. This water flux increase is attributed to the thinner velocity and temperature boundary layers at higher flow rates and therefore there is a more uniform temperature at channels to reduce thermal resistance and increase the water flux production. The feed water leaves the channel at higher temperatures and permeate outlet temperatures have lower values due to the less temperature change at a higher flow rate.

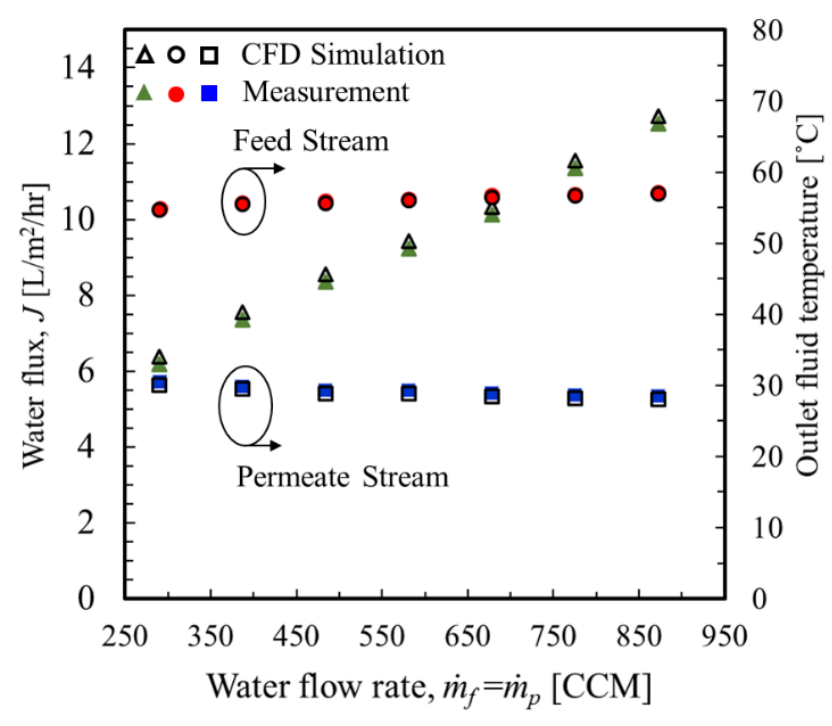

Figure 4.6 Effects of feed and permeate flow rates on water flux, outlet feed and permeate temperatures

\subsubsection{Membrane Distillation Scaling}

The MD experiments were started with $80 \%$ of saturation condition at temperatures of $75^{\circ} \mathrm{C}, 60^{\circ} \mathrm{C}$ and $45^{\circ} \mathrm{C}$ which are the feed inlet temperatures for three individual experiments. In these experiments, there is no makeup water to keep the concentration constant in the feed water and therefore, the concentration of the feed water increases by 
losing water at the feed water loop. It was observed two different regimes in the water flux behavior regarding the salinity. The first regime is a gradual decrease in the water flux due to an increase in the $\mathrm{NaCl}$ concentration resulting in the suppression of the vapor pressure at feed water. This regime was simulated by the CFD model and there is a good agreement between the experimental and numerical results.

In the second regime, it was observed that the water flux starts to drop sharply when the concentration of the feed water is about $90 \%$ of the saturation condition as shown in Fig. 4.7. It is attributed to the fact that in the saturation condition, the $\mathrm{NaCl}$ crystals were formed in the feed water and the deposition of crystals on the membrane surface leads to the blockage of the membrane pores. The continuous running MD operation eventually results in the zero water flux. The results show that the higher feed water temperature results in a higher decrease in the water flux. The sharp drop in the water flux was captured by the analytical correlation of Eq. (4.18). the relation between $C_{A}$ in Eq. (4.18) and feed inlet temperature was determined from the experimental data as $C_{A}=-1.5 \times 10^{-8}\left(T_{f, i^{-}}\right.$ $25)+1.64 \times 10^{-6}$. Figure 4.7 shows that the CFD simulation predicts the behavior of the water flux reduction when the saturation ratio is smaller than 0.9 while the analytical captures the sharp drop in the water flux when the saturation ratio is bigger than 0.9. 


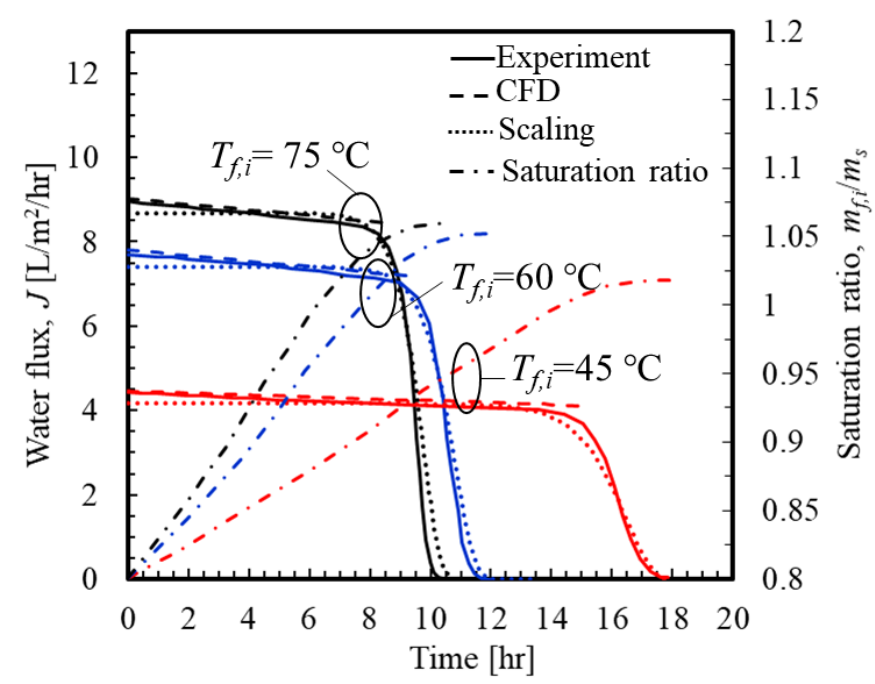

Figure 4.7 Water flux and saturation ratio versus time in MD operation

\subsubsection{Effects of Cooling Rates in Crystallization Process}

The brines of MD operations for three different feed inlet temperatures $\left(75^{\circ} \mathrm{C}, 60^{\circ} \mathrm{C}\right.$ and $45^{\circ} \mathrm{C}$ ) were cooled down to the ambient temperature by forced and natural cooling methods. For the forced cooling method, the cold water of the chiller was continuously circulated in the crystallizer reservoir. The variations of the temperature versus time are shown in Fig. 4.8. The results show that the forced cooling method is more efficient than natural cooling method and the rate of cooling is higher when the inlet feed temperature is higher. The filtered crystals were examined under the microscope (Fig. 4.9) to derive the distribution of the crystals as shown in Fig. 4.10. The crystallization process started with nucleation which is controlled by degree of super-saturation and continued with growth of nucleus which is manipulated by diffusion mechanism. The results demonstrate that the forced cooling method results in a smaller crystal size. This is attributed to the fact that the crystal nuclei have less time to grow to a larger size crystal, while in natural cooling, crystals are allowed to grow due to having more time. 
The criteria required for the ions $\left(\mathrm{Na}^{+}\right.$and $\left.\mathrm{Cl}^{-}\right)$to join the nucleate depends on the difference between the nucleus free energy and the solute free energy. If the energy of the nucleus is less than the solute, then nucleate starts to grow which occurs in super-saturation solution. In addition, homogeneous crystallization nucleation is governed by surface free energy and volume free energy. While a nucleus grows, the surface free energy increases but volume free energy decreases. The opposing effects of these two energy types provides a critical nucleus size, above which the crystal is stable. This critical nucleus size is smaller for higher super-saturation degree of the brine. Therefore, the smaller crystals are generated in the higher feed water temperatures with higher super-saturation degrees as illustrated in Fig. 4.10.

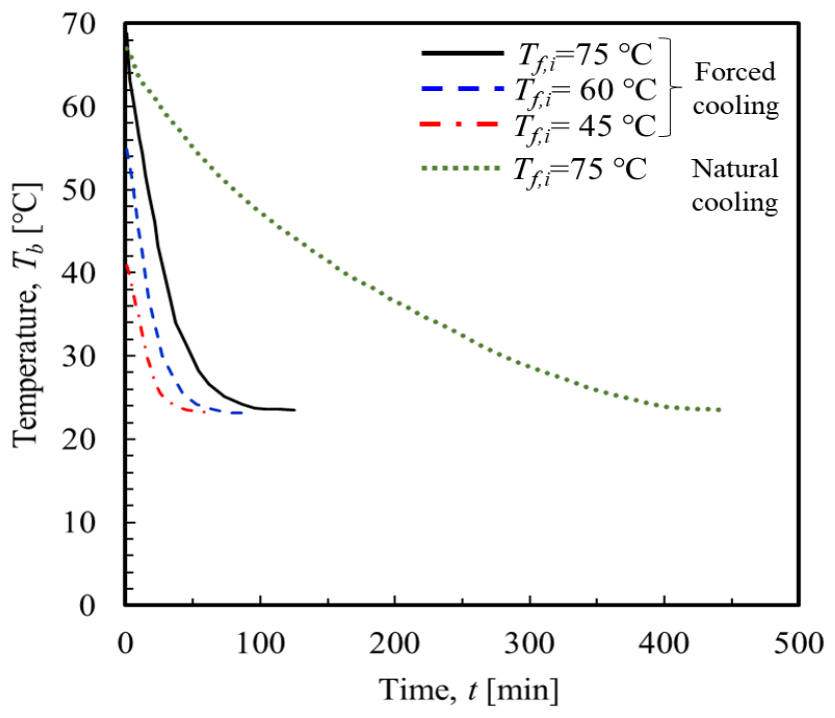

Figure 4.8 Brine temperature profiles in the crystallization process 

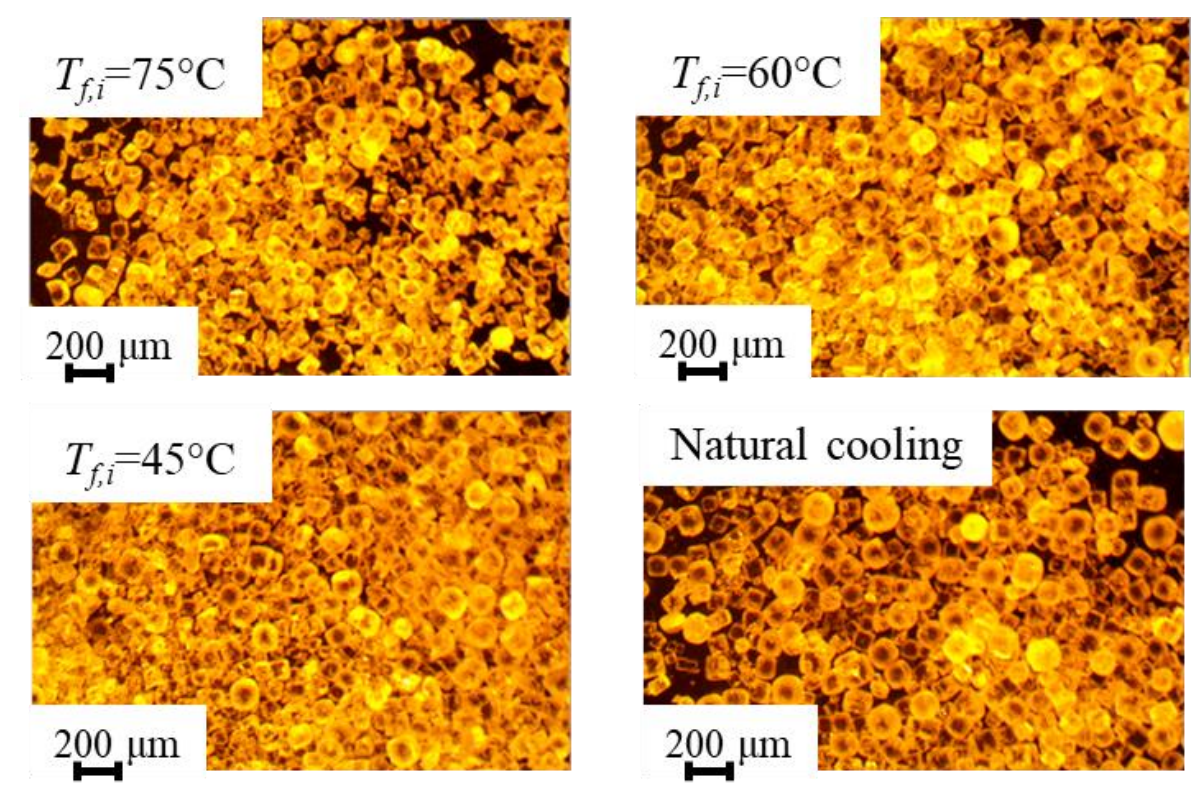

Figure 4.9 Microscopic images of $\mathrm{NaCl}$ crystals for natural and forced cooling methods

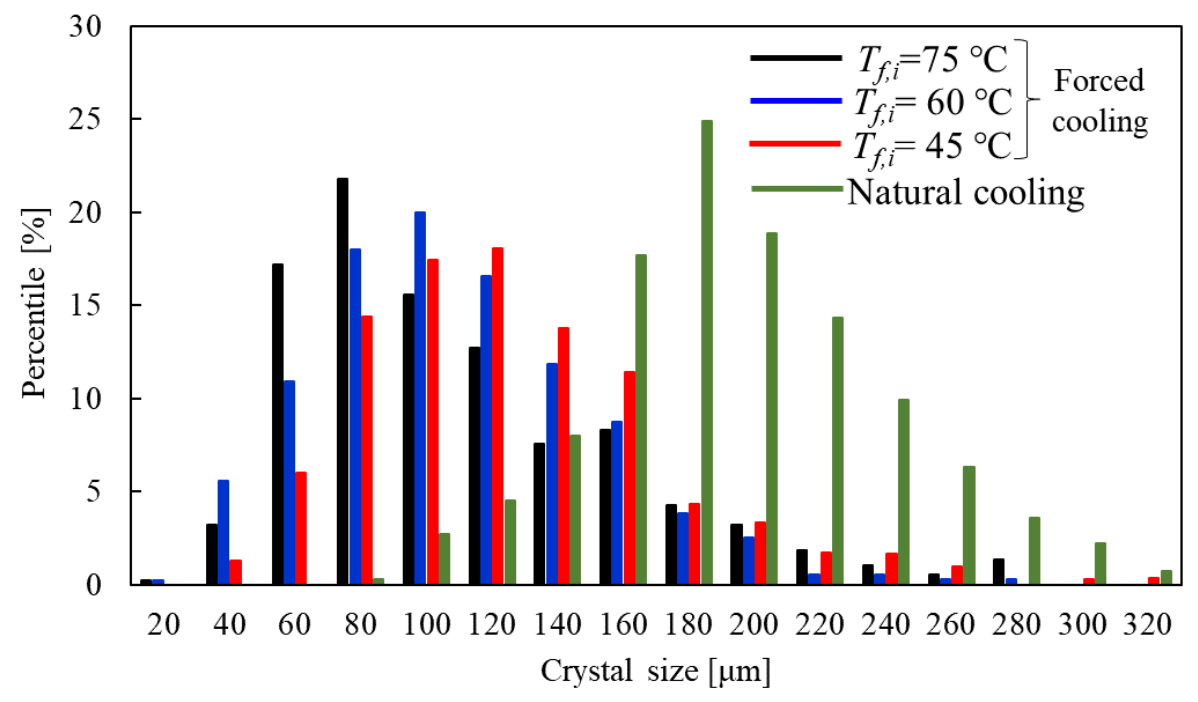

Figure 4.10 Distribution of salt crystal sizes for forced and natural cooling

\subsection{SUMMARY}

An experimental setup of membrane distillation crystallization was designed, and several experiments were performed. The experimental results showed that by increasing the $\mathrm{NaCl}$ concentration up to $90 \%$ of saturation condition, the water flux decreased due to 
the suppression of vapor pressure in the feed water and the CFD model results showed good agreement with experimental results. At the $\mathrm{NaCl}$ concentration higher than $90 \%$ of saturation condition, the $\mathrm{NaCl}$ crystals deposited on the membrane surface and the water flux dropped sharply and the analytical model predicted the crystal deposition effect on water flux performance for different inlet feed temperatures. In the crystallization process, the size of $\mathrm{NaCl}$ crystals was affected by the cooling rates and the higher cooling rates delivered the smaller crystal sizes. 


\section{CHAPTER 5: CARBON NANOTUBE DIRECT CONTACT MEMBRANE DISTILLATION}

Carbon Nanotubes (CNTs) with the exceptional electrical, mechanical and thermal characteristics have inspired many researchers to use them for many applications such as nanosensors, energy storage, and drug delivery. The experimental $[50,51]$ and theoretical $[14,52]$ studies report that the water flow rate through the CNT is higher than the estimation of Hagen-Poiseuille correlation by several orders of magnitude. This extraordinary result of water transport through CNT inspired researchers to use CNTs as desalination membranes. Recently, Gethard et al. [12] showed that adding CNT to polyvinylidene fluoride (PVDF) membrane is effective to enhance the water flux by $85 \%$. They postulated that the increase in the water flux might be attributed to fast diffusion transport of water vapor through the smooth [13] and hydrophobic CNT [14]. Most recently, An et al. illustrated that adding CNT to polyvinylidene fluoridehexafluoropropylene (PVDF-HFP) membrane results in more than $100 \%$ improvement in water flux of membrane distillation [15]. This improvement was explained by CNT surface diffusion. Furthermore, the buckypaper membrane, a CNT-based membrane, was used for a direct contact membrane distillation to achieve $99 \%$ salt rejection $[16,17]$.

In this paper, the water vapor transport through a single CNT in direct contact membrane distillation (DCMD) was investigated using non-equilibrium molecular dynamics (NEMD) simulation to study the effectiveness of the CNT membrane for the water distillation. The DCMD system used for the simulation consists of two reservoirs for feed and permeate water connected via a single CNT. The temperature of the feed reservoir was maintained at a higher temperature than that of the permeate reservoir to establish a 
difference in the saturation pressure in the hot and cold reservoirs and create a water vapor flow through the CNT tube. The distillation performance of the CNT DCMD system was calculated by NEMD simulation for different CNT diameters and lengths, atomic attraction strength between water molecules and CNT, and operating conditions such as system temperature, temperature difference between the feed (hot) and permeate (cold) reservoirs, and sodium chloride $(\mathrm{NaCl})$ concentration in the feed reservoir. In this study, liquid flooding in a CNT using a piston-driven flow was also investigated to determine the onset pressure of flooding and contact angle for different CNT diameter.

\subsection{NUMERICAL METHOD}

The extended Simple Point Charge (SPC/E) water model is used in this study to simulate water molecules. The SPC/E model, besides its simplicity, predicts well the properties of water compared with other water models such as TIP3P, TIP5P, and TIP4P [53]. It is specifically shown that water surface tension predicted by the SPC/E model shows the best agreement with the experimental results among other water models [54]. In the CNT membrane distillation, the surface tension of the water plays a key role on the creation of the water meniscus at the opening of the CNT, and therefore, the SPC/E model is the best choice to simulate the interactions between water molecules for the CNT membrane distillation system. The SPC/E model developed by Berendsen et al. [55] can handle the interaction between Oxygen atoms using Lennard-Jones potential and the electrostatic forces due to the charge of Oxygen and Hydrogen atoms. The SHAKE [56] program is used to maintain the specified bonds and angles of the water molecules in the equilibrium which are $0.1 \mathrm{~nm}$ and $109.47^{\circ}$ for $\mathrm{H}-\mathrm{O}$ bond length and $\mathrm{H}-\mathrm{O}-\mathrm{H}$ angle, 
respectively. The ions of sodium and chloride are also modeled as charged Lennard-Jones particles [57] which are expressed as

$$
\varphi_{w, i j}=4 \varepsilon_{i j}\left[\frac{\sigma_{i j}}{r_{i j}^{12}}-\frac{\sigma_{i j}}{r_{i j}^{6}}\right]+\sum_{i} \sum_{j} \frac{q_{i} q_{j}}{r_{i j}},
$$

where $\varepsilon$ and $\sigma$ are the Lennard-Jones parameters between atoms $i$ and $j$ are listed in Table $5.1[58,59] . q_{i}$ is the charge of the $i$ th atom. The cross Lennard-Jones interactions are modeled by the Lorentz-Berthelot rules, i.e., $\varepsilon_{i j}=\left(\varepsilon_{i}+\varepsilon_{j}\right)^{1 / 2}$ and $\sigma_{i j}=\left(\sigma_{i}+\sigma_{j}\right) / 2$, which are used to determine the potential parameters between different atom types and the cutoff distance is $15 \AA$. The long-range coulombic interactions of the charged atoms are determined by the particle-particle particle-mesh (PPPM) method [60].

Although stable single-walled CNTs with diameter higher than $1.5 \mathrm{~nm}$ cannot easily be fabricated, the interaction between water and carbon is the same for single-wall and multi-wall CNTs due to the short-range interactions between carbon and water. Also, carbon atoms in single-walled CNT are located in a fixed position for computational efficiency for this study $[52,61]$. 
Table 5.1 The Lennard-Jones parameters and charges of the simulated atoms

\begin{tabular}{lccc}
\hline \hline \multicolumn{1}{c}{ Parameters } & $\varepsilon[\mathrm{kcal} / \mathrm{mole}]$ & $\sigma[\AA]$ & $q[\mathrm{e}]$ \\
\hline \hline Oxygen-Oxygen (O-O) & 0.1553 & 3.1660 & -0.8476 \\
\hline Hydrogen-Hydrogen (H-H) & 0.0000 & 0.0000 & +0.4238 \\
\hline Carbon-Carbon (C-C) & 0.0700 & 3.5500 & - \\
\hline Sodium ion- Sodium ion $\left(\mathrm{Na}^{+}-\mathrm{Na}^{+}\right)$ & 0.1247 & 2.8760 & +1.0000 \\
\hline Chloride ion- Chloride ion $\left(\mathrm{Cl}^{-}-\mathrm{Cl}^{-}\right)$ & 0.1247 & 3.7850 & -1.0000 \\
\hline \hline
\end{tabular}

Figure 5.1 shows the domain of the CNT DCMD system used for the NEMD simulation. The CNT DCMD system is composed of a CNT connecting two water reservoirs: each confined by two graphene sheets. For flooding simulation, water molecules are only placed in the top reservoir with a size of $L_{x} \times L_{y} \times L_{z}=12 \mathrm{~nm} \times 12 \mathrm{~nm} \times 4 \mathrm{~nm}$ [Fig. 5.1(a)]. The top graphene sheet of the top reservoir works as a piston and is moved down by a certain incremental distance to squeeze the water molecules out of the top reservoir [Fig. 5.1(b)]. After each incremental movement, the water molecules are allowed to reach equilibrium for $1 \mathrm{~ns}$ with the NVT ensemble at $300 \mathrm{~K}$. In the equilibrium, the reservoir pressure is calculated based on the total force exerted by water molecules on the top graphene sheet (piston) as follows

$$
\begin{aligned}
& \mathbf{F}_{p}=-\sum_{i=1}^{n_{g}} \sum_{j=1}^{n_{w}}-\frac{d \varphi_{i j}}{d r_{i j}}, \\
& p_{p}=\frac{\mathbf{F}_{p, z}}{A_{p}},
\end{aligned}
$$


where $\varphi_{i j}$, and $r_{i j}$ are the Lennard-Jones (LJ) potential and distance between atoms $i$ and $j$. $\mathbf{F}_{p}$ and $\mathbf{F}_{p, z}$ are the total and $z$-directional force applied on the piston by water molecules. $p_{p}$ and $A_{p}$ are the pressure and area of the piston. On a large scale, the Young-Laplace equation defines the discontinuity of the pressure across the meniscus (vapor/liquid interface) inside the capillary tube.

For a sufficiently small tube diameter, the Young-Laplace equation can be simplified as

$$
p_{c}=\frac{4 \gamma \cos \theta}{d_{C N T}} \text {, }
$$

where $p_{c}$ is the pressure imbalance across the liquid interface which is called capillary pressure. $\gamma$ denotes water surface tension. $\theta$ is the contact angle and $d_{C N T}$ is the CNT diameter.

For the water distillation operation, the bottom and top reservoirs with the same volume of $L_{x} \times L_{y} \times L_{z}=12 \mathrm{~nm} \times 12 \mathrm{~nm} \times 4 \mathrm{~nm}$ are filled with water molecules as shown in Fig. 5.1(c). An NVT ensemble is applied to the water molecules in the reservoirs to maintain them at preset hot and cold temperatures in the respective bottom and top reservoirs. Due to the temperature (saturation pressure) difference between the reservoirs, the water liquid molecules evaporate at the opening of the CNT in the hot reservoir, flow through the CNT and condense in the cold reservoir. At the beginning of the MD simulation, the water liquid molecules inside the reservoirs were equilibrated for $1 \mathrm{~ns}$ to 
reach the preset temperatures. The simulation continued for $20 \mathrm{~ns}$ with a time step of $1 \mathrm{fs}$ to calculate water vapor flux through the CNT.

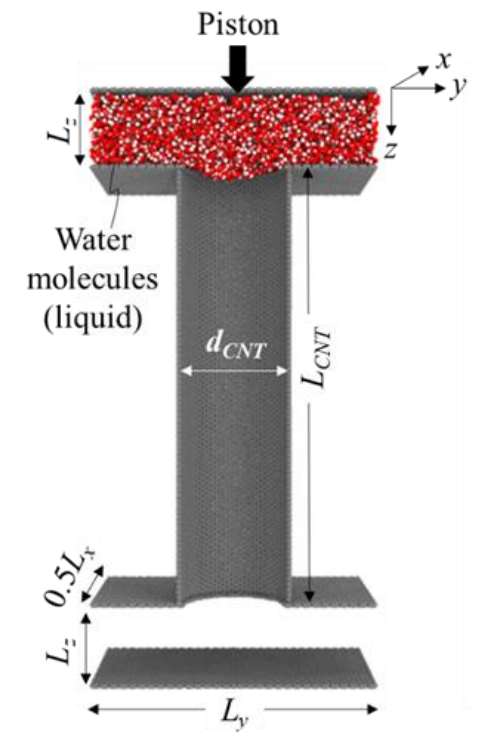

(a)

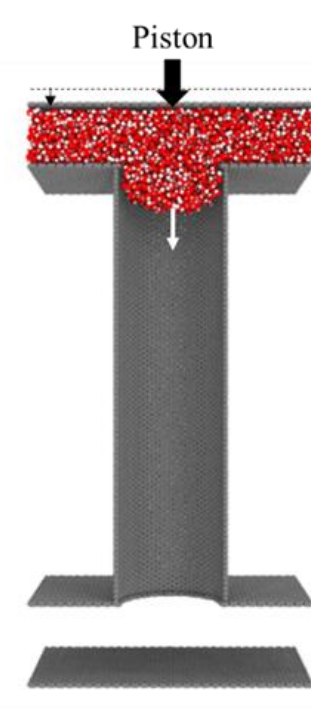

(b)

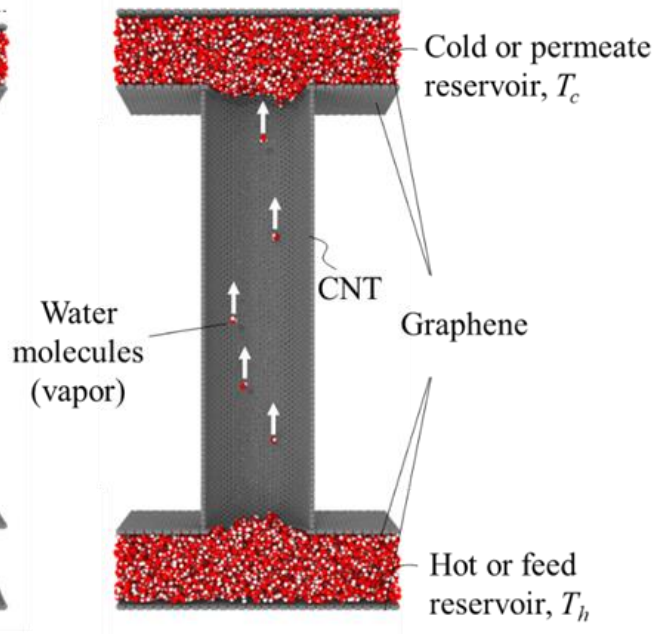

(c)

Figure 5.1 CNT membrane (a) before and (b) after flooding condition, and (c) CNT DCMD system showing water vapor flow between the hot and cold reservoirs

In the CNT DCMD, the partial pressure difference between two water reservoirs is the driving force for water vapor flow through the CNT. Since the CNT diameter is smaller than the mean free path of the water vapor molecules, the water vapor transport through the CNT is at Knudsen diffusion regime, where the water vapor molecules collisions with the CNT wall are more dominant than collisions between the water vapor molecules. The mass flux of water molecules in a cylindrical tube in the Knudsen regime is given by [62]

$$
J_{M D}=\frac{D_{M D}}{k_{\mathrm{B}} T_{\text {ave }} L_{C N T}} \Delta p_{s a t},
$$

where $J_{M D}$ is the water vapor flux. $D_{M D}, k_{\mathrm{B}}, T_{\text {ave }}$, and $L_{C N T}$ are the mass transport diffusivity, Boltzmann constant, average temperature of the system and length of the CNT, 
respectively. $\Delta p_{\text {sat }}$ is the saturation pressure difference between the hot (feed) and cold (permeate) reservoirs. $D_{M D}$ is directly calculated by Eq. (5.5) using $J_{M D}$ and $\Delta p_{\text {sat }}$ from the MD simulation.

A theoretical equation for the mass transport diffusivity for the Knudsen regime is formulated using the Kinetic Theory [63] and the Knudsen diffusivity is given by

$$
D_{\mathrm{Kn}}=\frac{4 d_{C N T}}{3} \sqrt{\frac{N_{\mathrm{A}} k_{\mathrm{B}} T_{\text {ave }}}{2 \pi M_{w}}},
$$

where $d_{C N T}, N_{\mathrm{A}}$, and $M_{w}$ are the diameter of CNT, Avogadro number, and water molecular weight, respectively. It is noteworthy that for the Knudsen diffusivity $\left(D_{\mathrm{Kn}}\right)$, all molecular collisions are assumed to be purely diffuse, and therefore the tangential momentum of the molecules is completely lost after a collision.

Smoluchowski modified the Knudsen correlation and postulated that a collision can be either diffusive or specular. The Tangential Momentum Accommodation Coefficient (TMAC) is defined as a ratio of diffusive collisions to total collisions in the system and is given by

$$
f_{M D}=\frac{v_{t, i}-v_{t, r}}{v_{t, i}}
$$

where $v_{t, i}$ and $v_{t, r}$ are the average tangential velocities of impinging and reflected water molecules respectively, which are obtained from MD simulation. $f_{M D}=1$ means that the collision is diffuse, i.e., total loss of the tangential velocity after a collision. $f_{M D}=0$ 
indicates that the collision is specular, i.e., no loss of the tangential velocity after a collision. For the combined collisions of the diffuse and specular types, water molecules existing within the cut-off distance from the carbon atoms of the CNT are attracted to the potential well and lose the kinetic momentum to the carbon atoms for a certain residence time before leaving the potential well beyond the cut-off distance. The Smoluchowski diffusivity is the modified Knudsen diffusivity using the TMAC [63] and is given by

$$
D_{\mathrm{Sm}}=D_{\mathrm{Kn}}\left(\frac{2-f_{\mathrm{MD}}}{f_{\mathrm{MD}}}\right) \text {, }
$$

Besides the transport diffusivity occurring inside the CNT, there is a translational self-diffusion of water molecules inside the water reservoir and the self-diffusion coefficient can be determined from the mean square displacement of water molecules as follows

$$
D_{\text {H2O }}=\lim _{t \rightarrow \infty} \frac{\left\langle(\Delta r)^{2}\right\rangle}{6 \Delta t},
$$

where $\Delta r$ is the displacement in the water molecule's coordinates after a time interval of $\Delta t$. The angle bracket denotes the average displacement over all the water molecules.

\subsection{MODEL VALIDATION}

To validate the MD simulation results from this study, the MD-simulated mass diffusivity of the water vapor molecules flowing through a single CNT was compared with available experimental results in literature. Bui et al. [64] used a nano-scale membrane made of vertically-aligned CNTs (average diameter of $3.3 \mathrm{~nm}$ and average length of 25 $\mu \mathrm{m})$ for water vapor diffusion through the CNTs between two nitrogen streams with 
different water vapor concentrations. Figure 5.2(a) illustrates the pressure profile (not scaled) across the CNT membrane showing the vapor and saturation (liquid) pressure differences $\left(\Delta p_{v}, \Delta p_{s a t}\right)$ and capillary pressure changes $\left(\Delta p_{c, h}, \Delta p_{c, c}\right)$ across the liquid/vapor interfaces. For a fair comparison with the experimental results for the pure water vapor transport, the saturation pressure difference $\left(\Delta p_{s a t}=\Delta p_{v}-\Delta p_{c, h}+\Delta p_{c, c}\right)$ in Eq. (5.5) which was commonly used for the mass flux calculation for the membrane distillation including the capillary pressure effect across the vapor/liquid interfaces, was replaced with the vapor pressure difference $\left(\Delta p_{v}\right)$ of the water vapor flow through the CNT which is the condition used in the experiment in literature [64]. The water vapor pressure was calculated using the saturation pressure by the Kelvin equation [65] as below,

$$
p_{v}=p_{s a t}(T) \exp \left(-\frac{4 \gamma V_{m} \cos \theta_{c}}{d_{C N T} R_{g} T}\right)
$$

where $p_{v}$ is the vapor pressure and $p_{s a t}$ is the saturation (liquid) pressure which are separated by the vapor/liquid interface. $\gamma, V_{m}, d_{C N T}$, and $R_{g}$ are the surface tension and molar volume of water, CNT diameter, and gas constant. $T$ denotes the temperature of water vapor. The capillary pressure jump and drop across the vapor/liquid interfaces are calculated by

$$
\Delta p_{c}=p_{s a t}(T)-p_{v}=p_{s a t}(T)\left[1-\exp \left(-\frac{4 \gamma V_{m} \cos \theta}{d_{C N T} R_{g} T}\right)\right],
$$


(a)

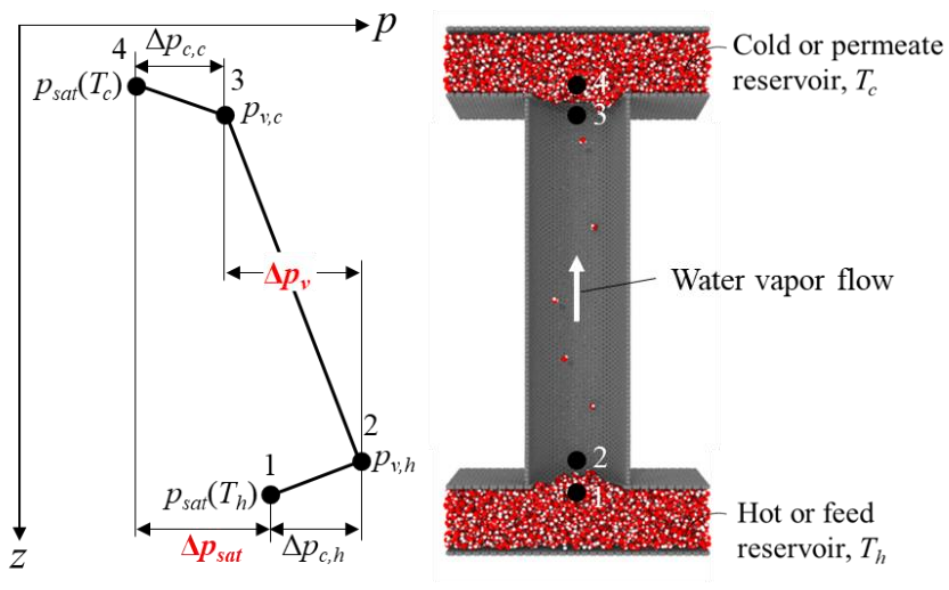

(b)

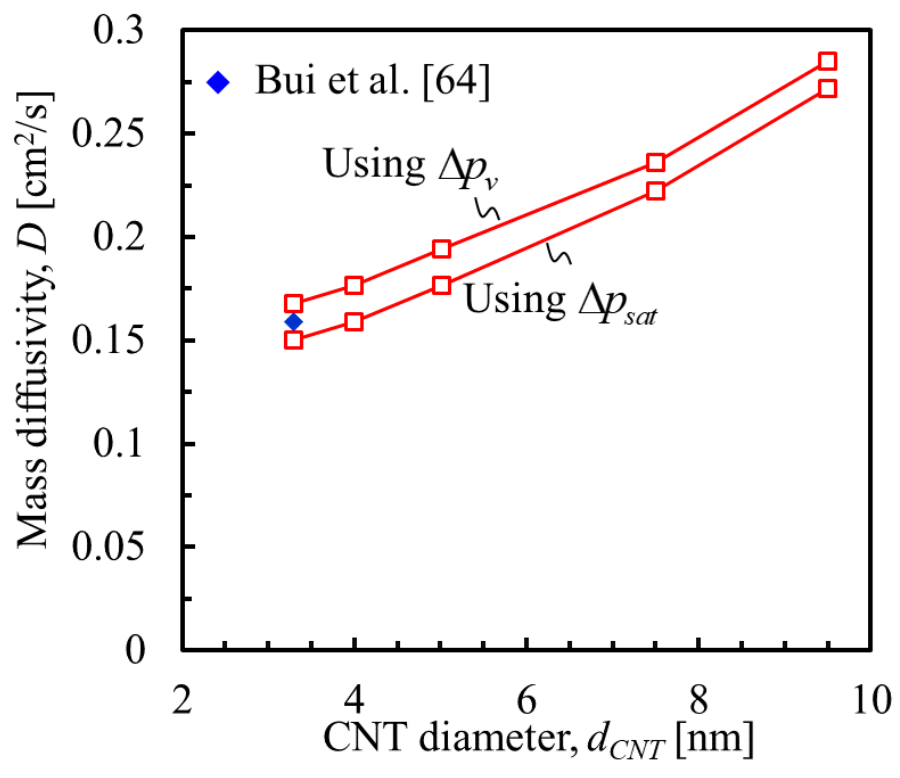

Figure 5.2 (a) Pressure profile across the CNT membrane (not scaled). (b) Comparison of the mass diffusivities from the MD simulation and experimental measurement [64]

Figure 5.2(b) shows the comparison of the mass diffusivities from the MD simulation and experimental measurement. The MD-simulated mass diffusivities were calculated using two kinds of the pressure differences between: (i) vapor pressures ( $\Delta p_{v}=$ 
$\left.p_{v, h}-p_{v, c}\right)$ and (ii) saturation pressures $\left[\Delta p_{s a t}=p_{s a t}\left(T_{h}\right)-p_{s a t}\left(T_{c}\right)\right]$. The MD-simulated mass

diffusivity based on the vapor pressure difference shows better agreement with the experimental measurement as shown in Fig. 5.2(b).

\subsection{RESULTS AND DISCUSSION}

\subsubsection{Onset of Liquid Flooding and Contact Angle}

Hydrophobicity of the CNT is critical to prevent the water liquid from entering (flooding) the CNT. If the capillary pressure of the convex meniscus formed at the entrance of the CNT is less than the water liquid pressure in the reservoir, the water liquid is pushed into the CNT causing an unwanted flooding. The pressure of the water liquid causing the flooding was calculated by molecular dynamics simulation. Also, the contact angle of the meniscus at the entrance of the CNT wall, which quantifies the hydrophobicity of the CNT with water and helps us to find the desirable operating conditions for the CNT DCMD system, was obtained on the verge of the flooding.

The liquid pressure of the top reservoir causing liquid flooding into the CNT was determined by moving the piston (upper wall of the top reservoir) down to pressurize the liquid reservoir and push the liquid into the CNT [Fig. 5.1(a,b)]. Figure 5.3 shows the water pressure variation with increasing piston displacement at three incremental movements $(\Delta \mathrm{z}$ $=0.01,0.02$, and $0.05 \mathrm{~nm})$. For the flooding simulation, the temperature of the water molecules was kept at $300 \mathrm{~K}$ using an NVT ensemble.

For the incremental movements of $0.02 \mathrm{~nm}$ and $0.01 \mathrm{~nm}$, the water pressure suddenly drops from its peak when the flooding occurs, while the incremental movement 
of $0.05 \mathrm{~nm}$ doesn't capture such a pressure drop. The rapid pressure drop is the indication of the flooding and right after the liquid pressure reaches the maximum pressure value as shown in Fig. 5.3. The flooding pressures of 0.02 and 0.01 incremental movements differ only $0.2 \%$. Therefore, the results of $0.01 \mathrm{~nm}$ incremental movement were used to find the flooding pressure $\left(p_{f}\right)$ for different CNT diameters as shown in Fig. 5.4(b).

Contact angle, which is an indicator of surface wettability, affects the water flooding in the CNT. The contact angle between water and CNT is at its maximum value at the onset of flooding, which is determined by the liquid-side angle [ $\theta_{c}$ in Fig. 5.4(a)] between the vertical line in the cutoff distance $\left(\sigma_{\mathrm{C}-\mathrm{o}}\right)$ from the inner wall of $\mathrm{CNT}$ and the tangent line of the vapor/liquid interface at the triple contact point. The vapor/liquid interface is defined by an isochoric profile with a value of $50 \%$ of water density (at 300 K). Figure 5.4(a) shows the isochoric profile and its curve fitting in the CNT with the diameter of $5.0 \mathrm{~nm}$. Figure 5.4(b) shows that as the CNT diameter increases, the contact angle increases because the coordination number of the water molecules at the vapor/liquid interface decreases due to higher distortion of the hydrogen bond of water molecules at the interface. The MD simulation results of the contact angle are in good agreement with the results from a reference [66]. The flooding pressure or liquid entry pressure (LEP) predictions from the MD simulations agree well with the results of the capillary pressure $\left(\Delta p_{c}\right)$ from the Young-Laplace equation [Eq. (5.4)] as shown in Fig. 5.5. 


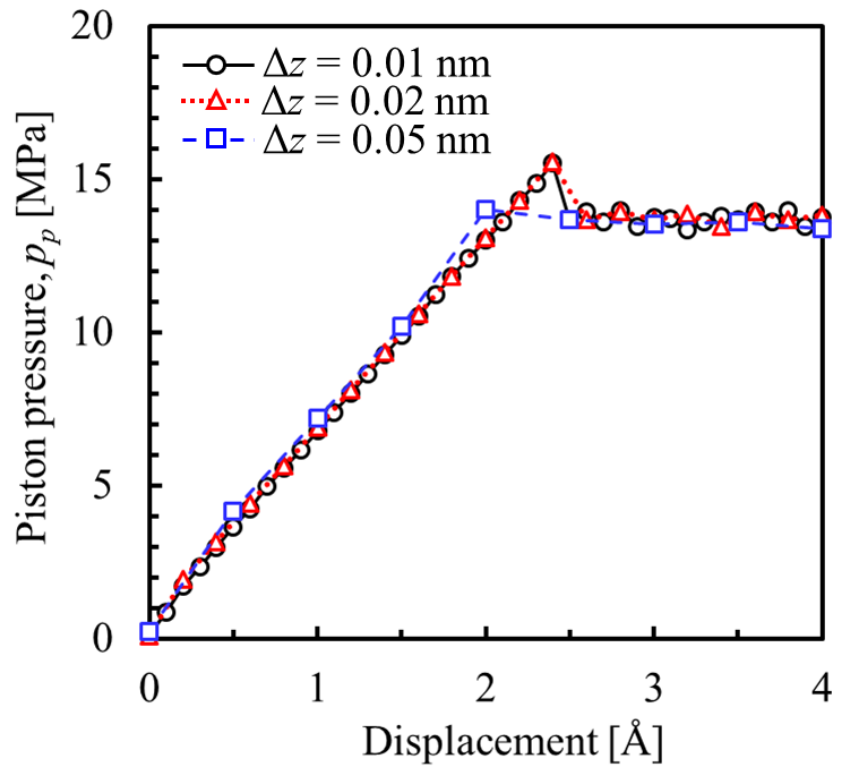

Figure 5.3 Effect of different incremental movement on showing water meniscus rupture

(a)

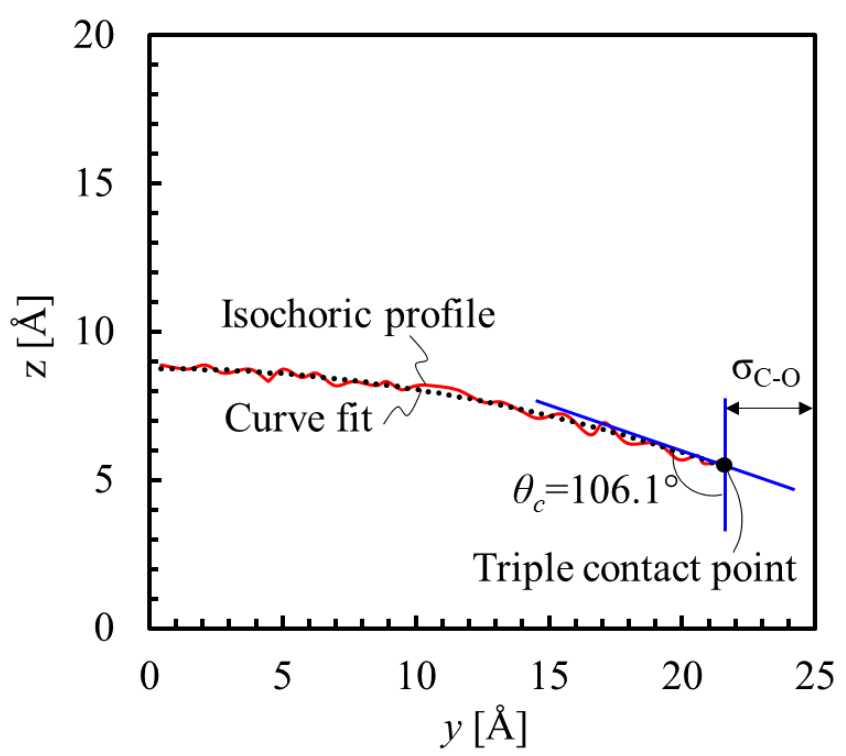


(b)

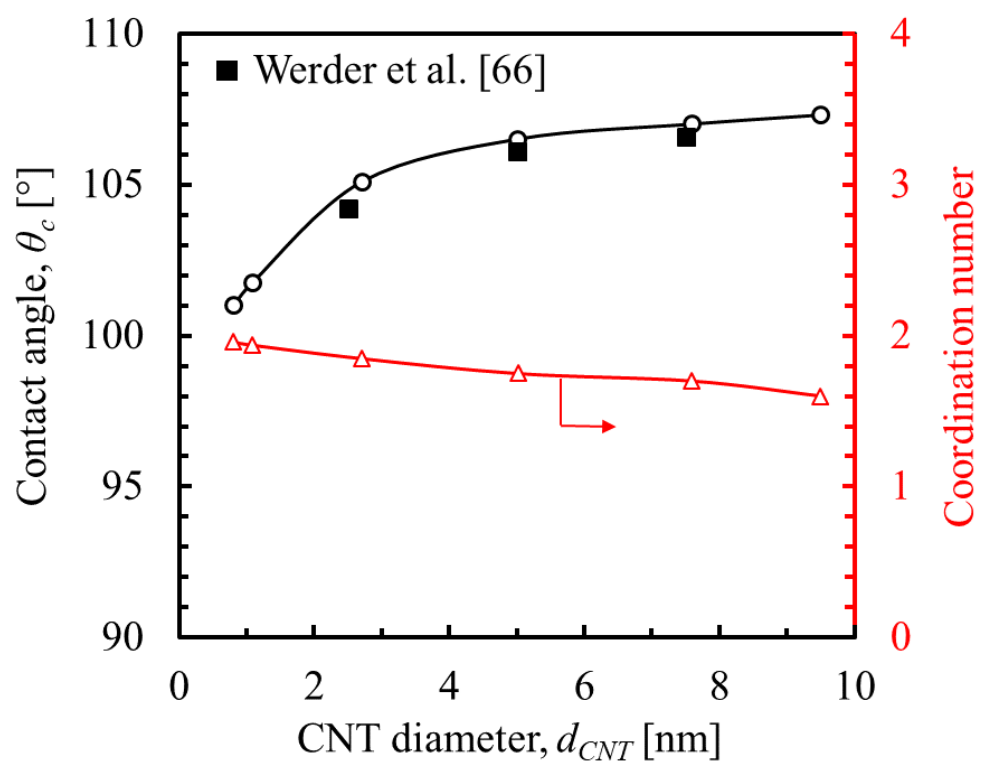

Figure 5.4 (a) Isochoric profile and curve fitting of $5.0 \mathrm{~nm}$ CNT diameter. (b) Variation of contact angle with respect to CNT diameter

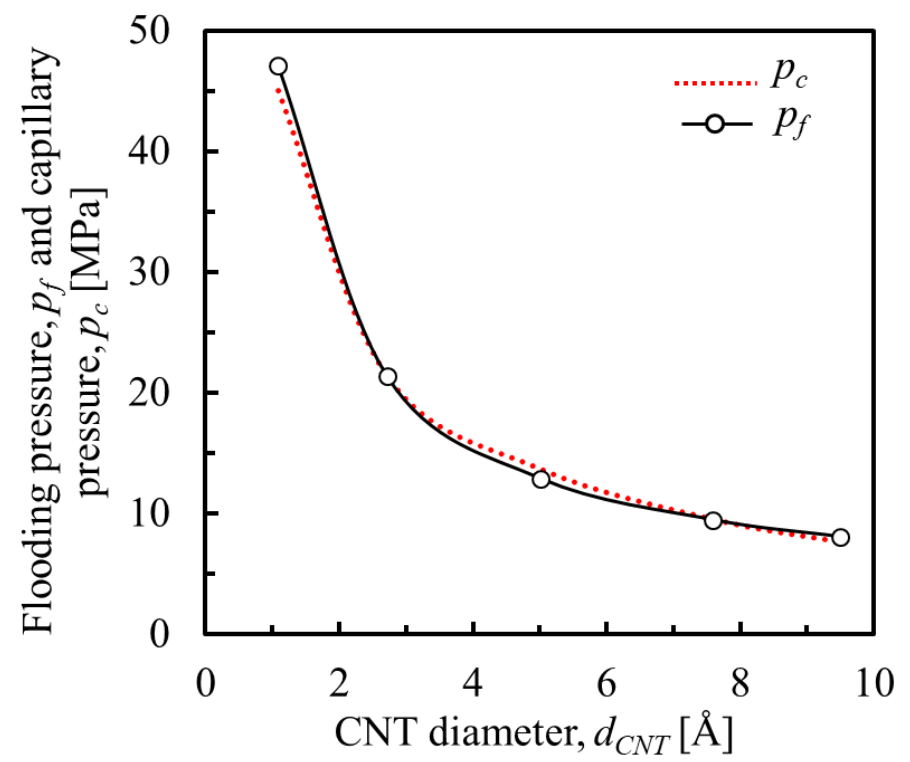

Figure 5.5 Variation of contact angle with respect to CNT diameter

In the following sections, the water distillation performance of the CNT membrane system was investigated by varying the operating conditions and CNT dimensions. The operating variables including system temperature (average of water reservoir temperatures) 
and temperature difference between the reservoirs control the pressure difference as the driving force for the water vapor flow through the CNT. The diameter and length of the CNT affect the frequency of the collision and interatomic force between the water molecules and CNT wall. Furthermore, the impact of attraction strength between water molecules and CNT on the water vapor transport was explored. Finally, the effect of sodium and chloride ions in the hot reservoir on water vapor distillation was investigated. The conditions and dimensions of the MD simulations are listed in Table 5.2.

Table 5.2 NEMD simulation conditions and dimensions

\begin{tabular}{cccccc}
\hline \hline Variables & \multicolumn{5}{c}{ Values } \\
\hline \hline$T_{\text {ave }}[\mathrm{K}]$ & 323 & $333^{*}$ & 343 & 353 & \\
\hline$T_{h}[\mathrm{~K}] / T_{c}[\mathrm{~K}]$ & $313 / 333$ & $323 / 343^{*}$ & $353 / 333$ & $343 / 363$ & \\
\hline$\Delta T^{\dagger}[\mathrm{K}]$ & 20 & 20 & 20 & 20 & \\
\hline \hline$\Delta T^{\dagger}[\mathrm{K}]$ & 10 & $20^{*}$ & 30 & 40 & \\
\hline$T_{h}[\mathrm{~K}] / T_{c}[\mathrm{~K}]$ & $328 / 338$ & $323 / 343^{*}$ & $318 / 348$ & $313 / 353$ & \\
\hline$T_{a v e}^{\dagger}[\mathrm{K}]$ & 333 & 333 & 333 & 333 & \\
\hline$d_{C N T}[\mathrm{~nm}]$ & 2.7 & $5.0^{*}$ & 7.5 & 9.5 & \\
\hline$L_{C N T}[\mathrm{~nm}]$ & 10 & $20^{*}$ & 50 & 100 & \\
$\left(L_{C N T} / d_{C N T}\right)$ & $(2)$ & $(4)$ & $(10)$ & $(20)$ & \\
\hline$\varepsilon_{\mathrm{C}-\mathrm{O}}[\mathrm{kcal} / \mathrm{mole}]$ & 0.1383 & $0.1127^{*}$ & 0.0576 & 0.0115 & 0.0023 \\
\hline$C[\mathrm{M}]$ & $0.0^{*}$ & 0.5 & 1.0 & 2.1 & \\
\hline \hline
\end{tabular}

* Baseline values used for the NEMD simulation.

${ }^{\dagger}$ Definitions: $T_{\text {ave }}=\left(T_{h}+T_{c}\right) / 2, \Delta T=T_{h}-T_{c}$.

\subsubsection{Effect of System Temperature}

The system temperature of the CNT DCMD greatly affects the water transport diffusivity and mass flux through the CNT. To investigate the effect of the system temperature on the distillation performance, the average reservoir temperature $\left(T_{\text {ave }}\right)$ was changed from $323 \mathrm{~K}$ to $353 \mathrm{~K}$, while maintaining a fixed temperature difference between 
the reservoirs, $\Delta T=20 \mathrm{~K}$. The saturation pressure in the hot reservoir is varied from 0.2 to 0.7 bar and in the cold reservoir ranges from 0.07 to 0.3 bar. The MD transport diffusivity $\left(D_{M D}\right)$ calculated by Eq. (5.5) using the results from the MD simulations for different system temperatures are shown in Fig. 5.6. As the system temperature increases, the water vapor mass flux increases due to the increased kinetic energy of water molecules. The trends of the MD transport, Smoluchowski and Knudsen diffusivities with the temperature are similar, but the MD transport diffusivity is roughly an order of magnitude higher than the Knudsen diffusivity and approximately $10 \%$ higher than the Smoluchowski diffusivity. The one order-of-magnitude difference between the MD transport and Knudsen diffusivities attributes to the inherent surface smoothness of the CNT which greatly reduces the tangential momentum loss of the collided water vapor molecules. The difference between the MD transport and Smoluchowski diffusivities is due to the small length-todiameter ratio of the CNT ( $L_{C N T} / d_{C N T}=4$ in Table 5.2), because the length-to-diameter ratio is assumed to be relatively large $\left(L_{C N T} / d_{C N T}>10\right)$ in the Smoluchowski model where the particles experience an infinitely large number of collisions in their path through the CNT. The TMAC $\left(f_{M D}\right)$ is much smaller than one, which indicates that specular collisions are more dominant than diffusive ones.

The TMAC decreases with increasing system temperature because the residence time of water molecules in the cut-off distance from the CNT atoms decreases due to the higher kinetic energy of water molecules, and therefore, the momentum exchange between the water molecules and CNT wall decreases. The permeability of the CNT membrane (1.8 $\times 10^{-5} \mathrm{liter} / \mathrm{m}^{2}$-s-Pa) for the baseline condition (Table 5.2) is two orders-of-magnitudes 
higher than a Polytetrafluoroethylene (PTFE) membrane $\left(1.7 \times 10^{-7} \mathrm{liter} / \mathrm{m}^{2}\right.$-s-Pa) used in our experimental work in Chapter 3. This huge enhancement attributes to the very high Knudsen diffusion of the CNT membrane (more than one order-of-magnitude) and the elimination of the molecular diffusion resistance between water vapor and air molecules occurring in the experiment using the PTFE membrane.

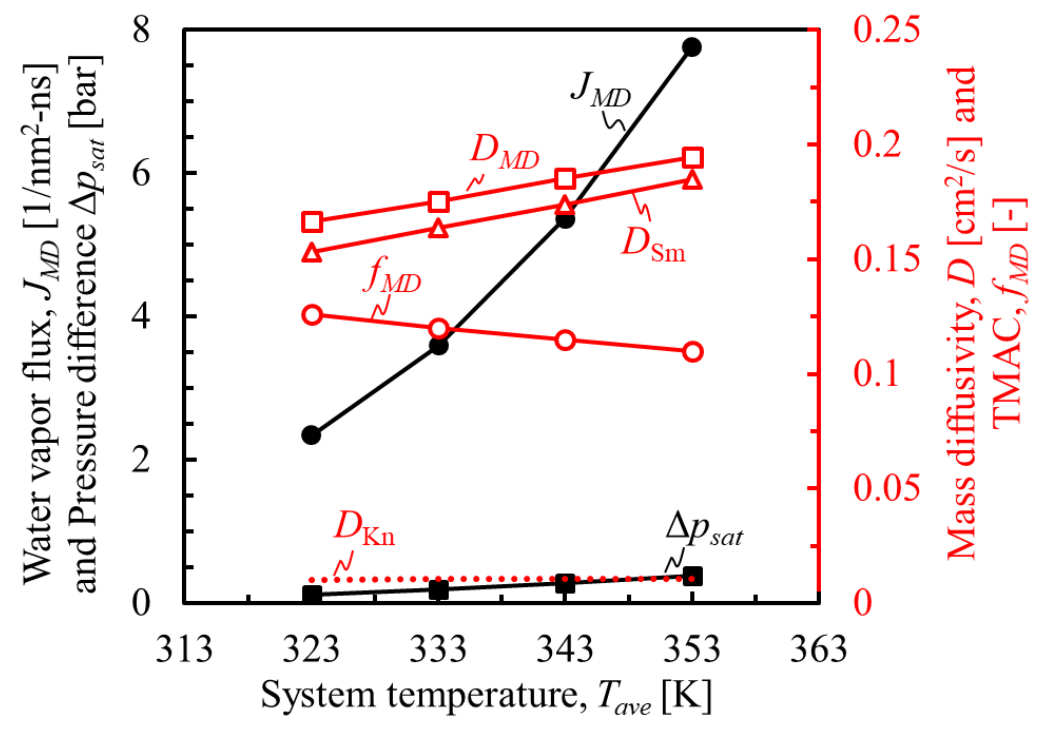

Figure 5.6 Variations of water vapor flux, diffusivity, and TMAC with respect to system temperature

\subsubsection{Effect of Reservoir Temperature Difference}

The temperature difference between the reservoirs determines the pressure difference between two reservoirs, which drives the water vapor through the CNT. The temperature difference ( $\Delta T=T_{h}-T_{c}$ ) between the reservoirs was varied with four different temperatures of 10,20,30, and $40 \mathrm{~K}$, while keeping the system temperature constant at the baseline value, i.e., $T_{a v e}\left[=\left(T_{h}+T_{c}\right) / 2\right]=333 \mathrm{~K}$ (Table 5.2). In Fig. 5.7, when the reservoir temperature difference increases, the water vapor flux increases approximately linearly due 
to the increase in the saturation pressure difference between the hot and cold reservoirs. However, the transport diffusivities and TMAC remain relatively constant because the average system temperature was kept constant.

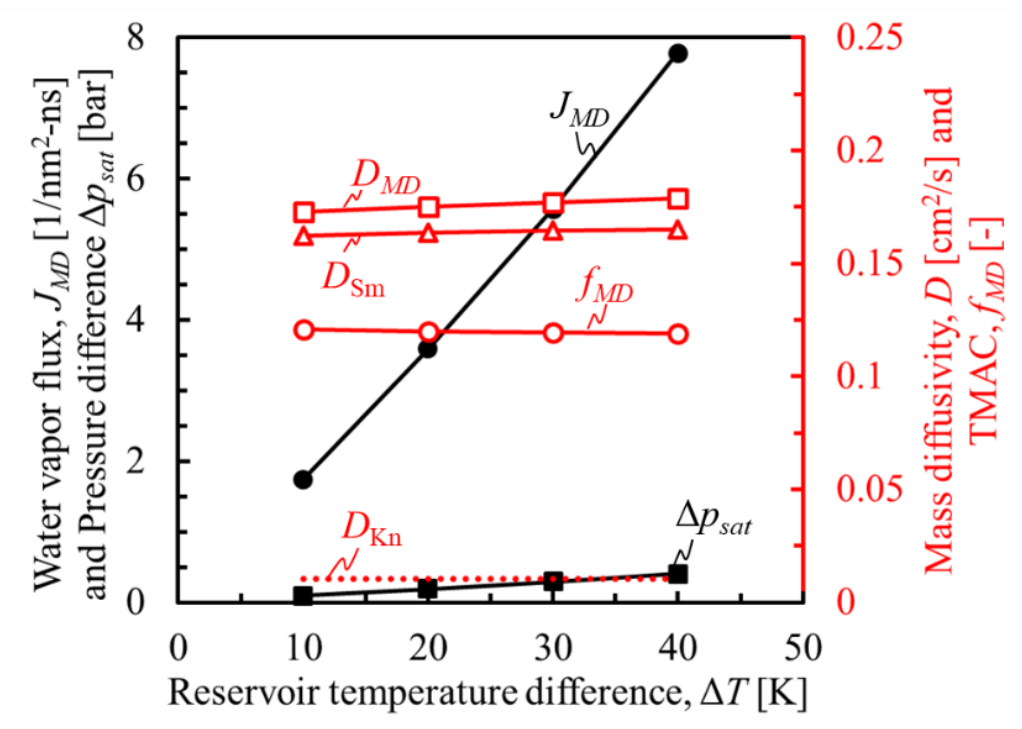

Figure 5.7 Effect of reservoir temperature difference on water vapor flux, diffusivity, and TMAC

\subsubsection{Effect of CNT Diameter}

There is some evidence that the phase transition of the confined water in the CNT deviates dramatically from the behavior of bulk water. Agrawal [67] experimentally showed that water at room temperature exists in a form of ice in a CNT with $1 \mathrm{~nm}$ diameter. Also, Chaban [68] reported that the boiling temperature of water is dramatically increased when the water molecules are trapped inside a CNT with a diameter of less than $2.85 \mathrm{~nm}$. In this study, we also found that, at the entrance of a CNT with a diameter of less than 1.5 $\mathrm{nm}$, the water evaporation hardly occurs. Due to the hydrophobicity of CNT, water molecules at the vapor/liquid phase are repelled by CNT, and therefore the water molecules 
need high thermal energy or temperature to overcome the repulsion and enter into the CNT. Therefore, the CNT diameters bigger than twice the cut-off distance ( $30 \AA$ ) should be chosen to initiate the evaporation of water molecules.

In Fig. 5.8, all the diffusivities (MD, Knudsen, and Smoluchowski) increase with the CNT diameter. This is due to the fact that by increasing the CNT diameter with a constant length, the probability of the collisions between the water vapor molecules and CNT wall decreases. The big deviation of the Knudsen diffusivity from the MD transport diffusivity is due to the assumption of purely diffuse collision for the Knudsen diffusivity resulting in too low diffusivity. The difference between the MD transport and Smoluchowski diffusivities is due to the fact that the Smoluchowski diffusivity is accurate for a large number of collisions of the water molecules with the CNT wall likely happening in a very long tube. It is worth noting that by decreasing diameter $\left(d_{C N T}\right)$ with a constant length $\left(L_{C N T}\right)$, the differences between the MD transport and Smoluchowski diffusivities values become smaller due to higher $L_{C N T} / d_{C N T}$. As shown in Fig. 5.8, the TMAC decreases with decreasing CNT diameter because more carbon atoms are involved to repel the water vapor molecules at their impacts on the CNT wall due to the higher curvature of the smaller CNT diameter. Thus, the residence time of the water vapor molecules in the cut-off distance decreases as shown in Fig. 5.8, and therefore, the momentum exchange between the water vapor molecules and CNT wall decreases. 


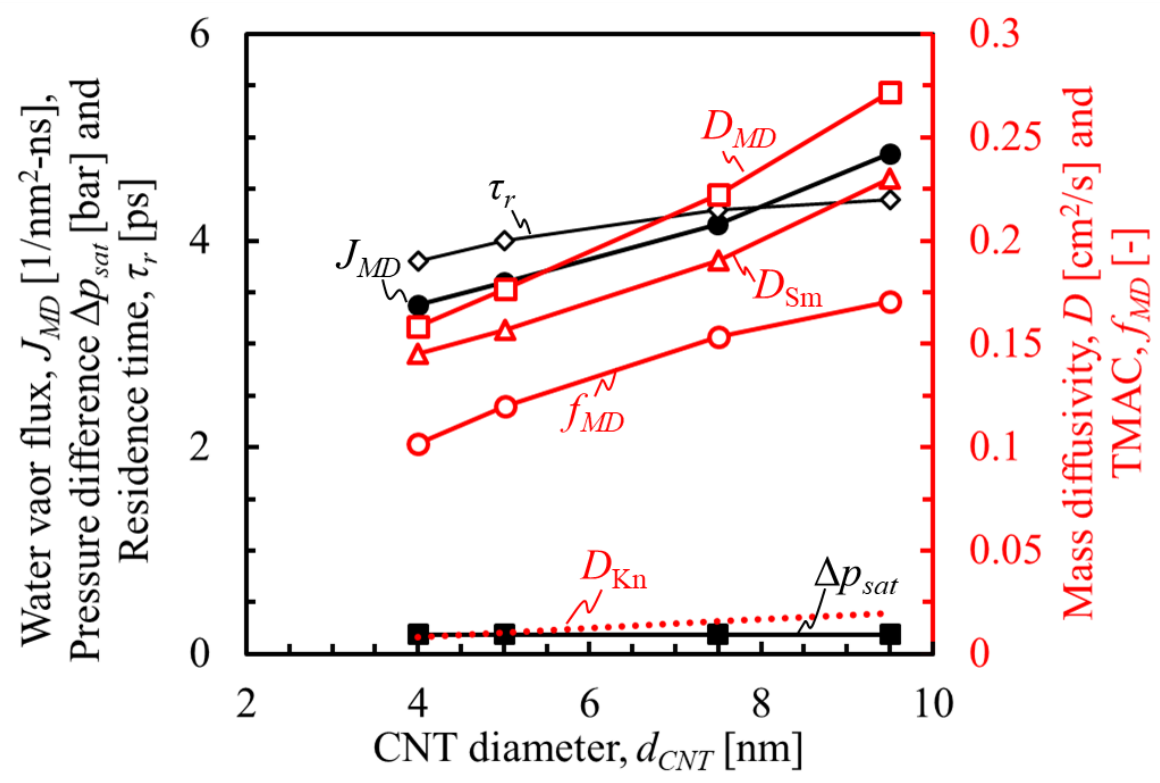

Figure 5.8 Variations of water vapor flux, diffusivity, TMAC, and collision residence time of water molecules with respect to CNT diameter

\subsubsection{Effect of CNT Length}

The effect of the CNT length $\left(L_{\mathrm{CNT}}\right)$ on the water vapor flux and mass transport diffusivities was investigated. In this section, the CNT length was increased, while the CNT diameter was kept constant at $d_{\mathrm{CNT}}=5.0 \mathrm{~nm}$ (Table 5.2). As shown in Fig. 5.9, as the CNT length increases, the water vapor flux exponentially decreases because of more collisions in longer CNT. Because of the same reason, the MD transport diffusivity decreases with increasing CNT length, while the Knudsen and Smoluchowski diffusivities remain constant which don't capture the effect of the length of the flow path.

For a generalized conclusion, an aspect ratio $\left(L_{\mathrm{CNT}} / d_{\mathrm{CNT}}\right), \mathrm{CNT}$ diameter to its length, was used in the second $x$-axis (top) of Fig. 5.9 to clearly show the critical aspect ratio for scale effect. The deviation of the MD transport diffusivity from the Smoluchowski diffusivity for smaller aspect ratios $\left(L_{\mathrm{CNT}} / d_{\mathrm{CNT}}<10\right)$ refers to "size effect" in the mass 
diffusivity calculation, while for higher aspect ratios $\left(L_{\mathrm{CNT}} / d_{\mathrm{CNT}}>10\right)$, the MD transport and Smoluchowski diffusivities are in good agreement. For smaller aspect ratios less than 10, a considerable number of water molecules pass through without collisions on the CNT wall which results in a higher mass diffusivity. However, for higher aspect ratios larger than 10 , the mass diffusivity is smaller due to less possibility of direct transport where collisions of water molecules with CNT wall are more frequent and the Smoluchowski correlation [Eq. (5.8)] is applicable. Note that in the Smoluchowski correlation, the length of the tube is not a variable because it is assumed to be large enough compared to the tube diameter which results in an insignificant direct transport (without collision). The results show that the TMAC ( $\left.f_{\mathrm{MD}}\right)$ is relatively constant for different CNT lengths because $f_{\mathrm{MD}}$ considers only the collision type (e.g. diffusive, specular) and mostly depends on local interaction forces between the water molecules and CNT atoms.

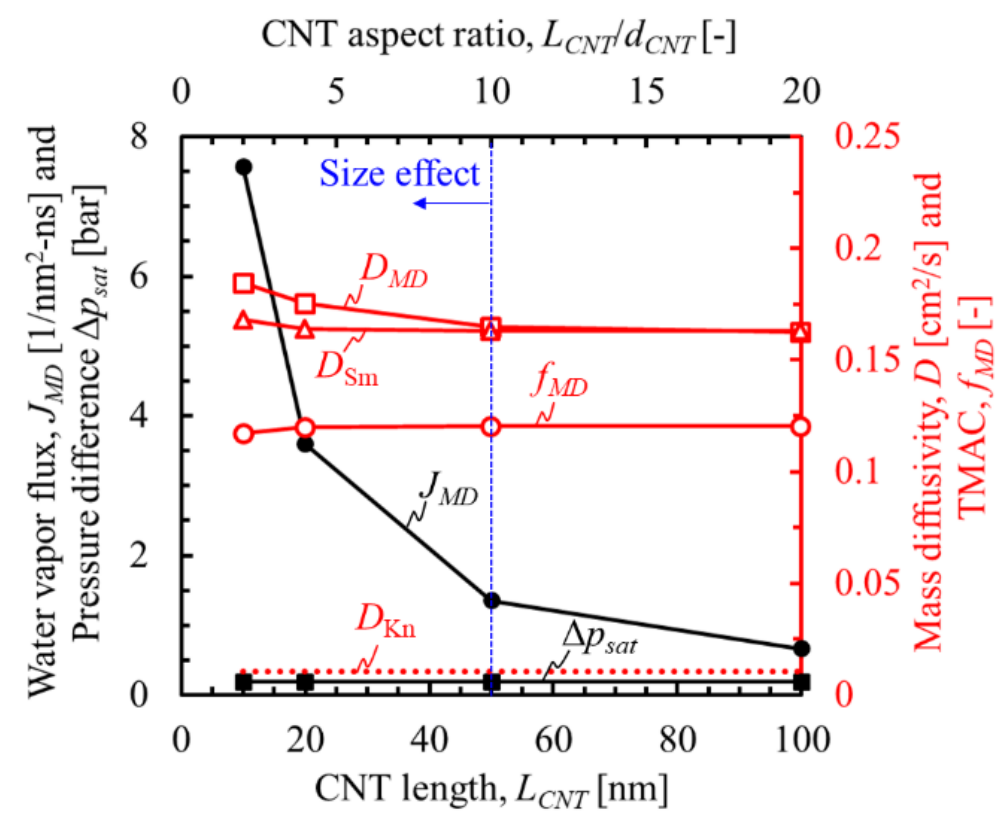

Figure 5.9 Effect of CNT length on water vapor flux, mass diffusivities, and TMAC 


\subsubsection{Effect of Interatomic Force Between CNT and Water}

To understand the Knudsen diffusion and water vapor transport, the energy constant $(\varepsilon \mathrm{c}-\mathrm{O})$ in the $\mathrm{LJ}$ potential between carbon atoms and water molecules varied from 0.0144 to $0.1500 \mathrm{kcal} / \mathrm{mol}$. Figure 5.10 shows that the water vapor flux and transport diffusivities decrease as the energy constant increases. This is attributed to the fact that the residence time $\left(\tau_{r}\right)$ of water molecules increases with increasing as shown in Fig. 5.10. For lower $\varepsilon_{\mathrm{C}-\mathrm{O}}$, water molecules striking the CNT wall instantaneously bounce back, while for higher $\varepsilon_{\mathrm{C}-\mathrm{O}}$, water molecules stay longer on the wall. As a consequence, more momentum of the water molecules is lost, resulting in a high TMAC as shown in Fig. 5.10. The Knudsen diffusivity cannot capture the collision mode between water molecules and CNT wall, while the Smoluchowski diffusivity implicitly considers it by using TMAC in the model. Figure 5.10 also shows that the difference between the MD transport and Knudsen diffusivities decreases as (attraction forces) between the water molecules and CNT increases. It means that the strong attraction between water molecules and CNT causes more momentum exchange between them and the collisions become more diffusive. In this study, the value of $\varepsilon_{\mathrm{C}-\mathrm{O}}$ cannot exceed the value of $\varepsilon_{\mathrm{O}-\mathrm{o}}$. The reason is that for higher than, the interactions between water molecules and CNT become stronger than that of between water molecules, and therefore there is no hydrophobic CNT behavior to avoid flooding. The deviation of the MD transport and Smoluchowski diffusivities is due to the size effect discussed in Section 5.3.4. 


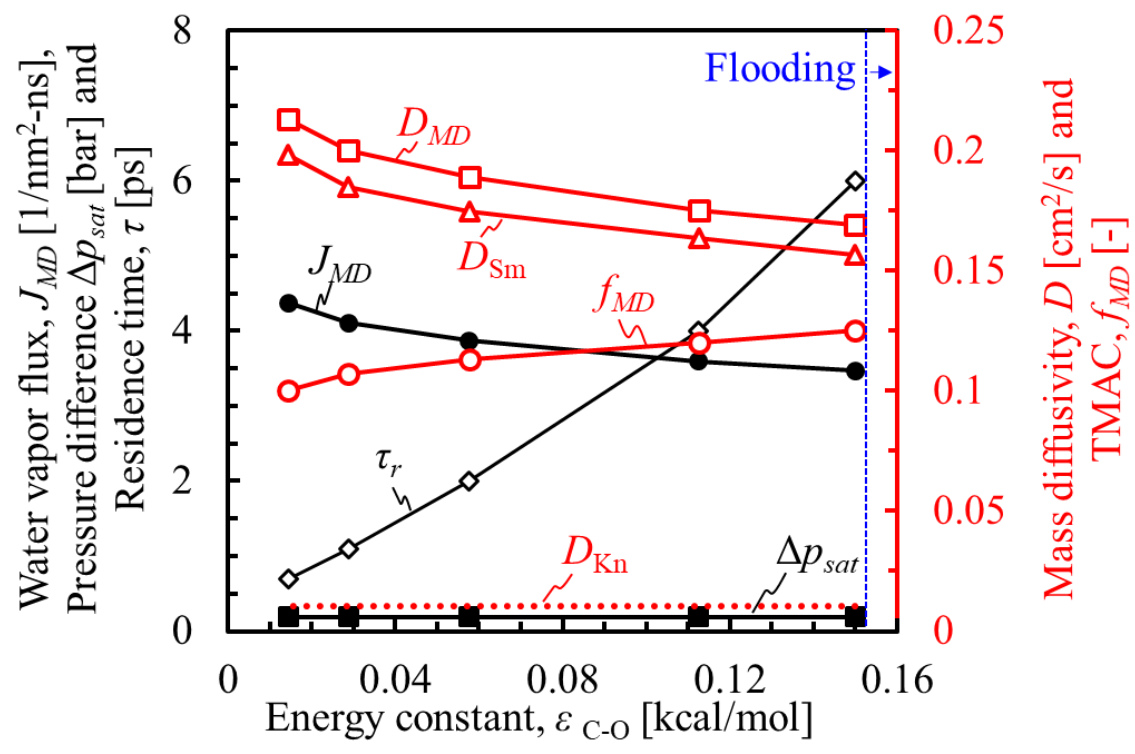

Figure 5.10 Variations of water vapor flux, diffusivity, TMAC and residence time with respect to energy constant of $\mathrm{LJ}$ potential between water molecules and carbon atoms

\subsubsection{Effect of Impurity}

It is interesting to investigate the effect of impurity (e.g., $\mathrm{NaCl}$ ) in the feed water for the membrane distillation. Figure 5.11 shows that the self-diffusion coefficient [ $D_{\mathrm{H} 2 \mathrm{O}}$ in Eq. (5.9)] of water molecules decreases with increasing $\mathrm{NaCl}$ concentration which attributes to the fact of increasing interactions between the water molecules and impurity ions. The presence of sodium chloride $(\mathrm{NaCl})$ in the feed water creates an ionic-dipole attraction between the water molecules and $\mathrm{NaCl}$ ions, which is stronger than the normal hydrogen bonds between water molecules, and therefore more energy is required to break the ion-dipole attraction, resulting in less evaporation of the water molecules at the meniscus. In addition, according to the Lorentz-Berthelot rules using the epsilons of the same atoms in Table 5.1, the epsilon in LJ potential between water molecules and carbon 
atoms is $0.1043 \mathrm{kcal} / \mathrm{mole}$ and the epsilon between the ions of impurity and carbon atoms is $0.0934 \mathrm{kcal} / \mathrm{mole}$. The smaller epsilon (i.e., interaction) between the ions and carbon atoms means the ions $\left(\mathrm{Na}^{+}\right.$and $\left.\mathrm{Cl}^{-}\right)$replacing the water molecules in the feed reservoir creates less pressure than that of pure water according to Eqs. (5.2-5.3). The decrease in the pressure of the feed reservoir causes a lower pressure difference between the reservoirs, and thus the water vapor flux decreases as illustrated in Fig. 5.11. However, the mass diffusivities and TMAC of water molecules inside the CNT does not change with concentration because the impurity stays only in the feed reservoir and does not enter into the CNT during the simulation time.

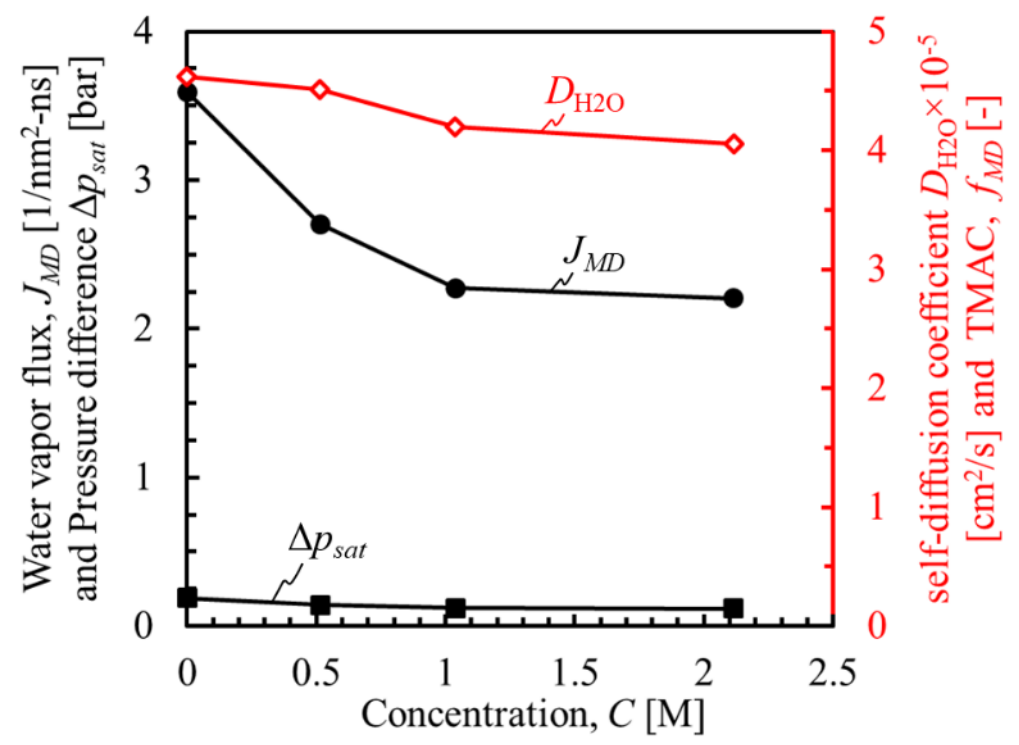

Figure 5.11 Effect of Sodium Chloride concentration on water vapor flux, diffusivity and TMAC

\subsection{SUMMARY}

Non-equilibrium Molecular Dynamics (NEMD) simulations were performed to investigate the water distillation performance of a CNT DCMD system. The MD 
simulation results showed that the water vapor transport through the CNT is enhanced by increasing the system temperature, reservoir temperature difference, and CNT diameter, while adverse results were observed by increasing the CNT length, attraction strength between water molecules and CNT, and sodium chloride concentration. The transport diffusivity increases with high system temperature, large CNT diameter with smaller length-to-diameter ratio, and small energy constant between water molecules and CNT. The permeability of the CNT membrane is two orders-of-magnitudes higher than a Polytetrafluoroethylene (PTFE) membrane due to much higher Knudsen diffusion of the CNT membrane (more than one order-of-magnitude) than that of the PTFE membrane. It is also found that the tangential momentum accommodation coefficient (TMAC) increases with CNT diameter and energy constant between water molecules and CNT, but it decreases with the system temperature. Since the TMAC is small for the CNT, the MD transport diffusivity is higher than the Knudsen diffusivity by an order of magnitude. The results illustrated that the size effect is critical in determining mass diffusivity for the length-to-diameter ratio smaller than ten. 


\section{CHAPTER 6: CONCLUSIONS}

The performance of the direct contact membrane distillation (DCMD) was analyzed in the multiple-module DCMD system using waste heat of the diesel engine by developing a thermal-hydraulic network model, in the single module DCMD by doing experiment and conducting computational fluid mechanics (CFD) simulation and in the carbon nanotube (CNT) DCMD by using molecular dynamics (MD) simulation.

In the multiple-module DCMD system, the numerical analysis using the onedimensional model of DCMDs and thermal-hydraulic connection with the diesel engine was developed. The analysis suggested that a flat plate heat exchanger was a viable choice for effective heat exchange from the diesel engine to the parallel DCMDs. It was found that the water distillate production was increased by increasing the flow rates and inlet temperature differences (ITD) of the feed and permeate streams. A high $\mathrm{NaCl}$ concentration in the feed stream lowered its vapor pressure and therefore decreased the water production. Shorter membranes produced larger water mass flux at higher thermal efficiency. Therefore, for a multiple-module DCMD system, a greater number of shorter membrane modules in a parallel arrangement was more effective in the water production, as long as the heat exchanger for diesel engine waste heat recovery was large enough.

In the single module DCMD, a CFD simulation of the DCMD system was validated by the experiment for different temperatures, flow rates of the feed and permeate streams, and salinity of the feed stream. It was found that a lower permeate water temperature, higher feed water temperature, lower salinity, and higher flow rates produced more distilled water. It was also found that distilled water production was influenced more by the feed temperature than the permeate temperature and a counterflow configuration produced more 
distilled water than that of the parallel flow configuration. The insertion of a mesh screen spacer in the flow channels, especially one with smaller spacing between mesh screen filaments, enhanced the convective heat transfer due to a converging-diverging flow around the screen spacer filaments and thus, reduced the temperature and concentration polarizations in the DCMD channels resulting in a higher distilled water production.

In the experiment, two different regimes in the water flux behavior were observed regarding the salinity. In the first regime, at the small salinities, there is a gradual decrease in the water flux due to the suppression of the vapor pressure at the feed water. This regime was simulated by the CFD model and there was a good agreement between experimental and numerical results. In the second regime, at high salinities near to saturation condition, there is a sharp drop in the water flux due to the deposition of the salt crystals on the membrane surface and block the area of the opening pores. This regime was captured by an analytical approach since CFD model results deviated from the experimental results.

A nanoscale CNT DCMD was simulated by non-equilibrium Molecular Dynamics (NEMD) to investigate the water vapor flux of the CNT DCMD. The NEMD simulation results showed that the permeability of the CNT membrane was two orders-of-magnitude higher than the Polytetrafluoroethylene (PTFE) membrane used in the experiment. Therefore, CNT is a promising substitution for PTFE polymer in DCMD. The water vapor transport through the CNT was enhanced by increasing the system temperature, reservoir temperature difference and CNT diameter, while adverse results were observed from the CNT length, attraction strength between water molecules and CNT, and sodium chloride concentration. The MD transport diffusivity increased with high system temperature, large CNT diameter and aspect ratio, and small energy constant between water molecules and 121 
CNT. It was also found that Knudson and Smoluchowski models were not capable to accurately predict the MD transport diffusivity inside CNT DCMD because the NEMD simulation result of the MD transport diffusivity was higher than Knudsen diffusivity by an order of magnitude and was higher than Smoluchowski diffusivity by $10 \%$ for small aspect ratio of CNT. 


\section{REFERENCES}

[1] M. Naim, Recent advances in desalination technologies: a review, acronym: TDWT.

[2] K.W. Lawson, D.R. Lloyd, Membrane distillation, Journal of membrane Science, 124 (1997) 1-25.

[3] D. Winter, J. Koschikowski, D. Düver, P. Hertel, U. Beuscher, Evaluation of MD process performance: effect of backing structures and membrane properties under different operating conditions, Desalination, 323 (2013) 120-133.

[4] J. Koo, J. Han, J. Sohn, S. Lee, T.-M. Hwang, Experimental comparison of direct contact membrane distillation (DCMD) with vacuum membrane distillation (VMD), Desalination and Water Treatment, 51 (2013) 6299-6309.

[5] Y. Shin, J. Choi, T. Lee, J. Sohn, S. Lee, Optimization of dewetting conditions for hollow fiber membranes in vacuum membrane distillation, Desalination and Water Treatment, 57 (2016) 7582-7592.

[6] F. Banat, N. Jwaied, Economic evaluation of desalination by small-scale autonomous solar-powered membrane distillation units, Desalination, 220 (2008) 566-573.

[7] E. Norouzi, C. Park, Numerical analysis of energy and mass flow of waste heat-driven direct contact membrane distillation system, Desalination and Water Treatment, 106 (2018) $40-50$.

[8] R.B. Saffarini, E.K. Summers, H.A. Arafat, Economic evaluation of stand-alone solar powered membrane distillation systems, Desalination, 299 (2012) 55-62.

[9] R. Sarbatly, C.-K. Chiam, Evaluation of geothermal energy in desalination by vacuum membrane distillation, Applied Energy, 112 (2013) 737-746. 
[10] F. Suárez, S.W. Tyler, A.E. Childress, A theoretical study of a direct contact membrane distillation system coupled to a salt-gradient solar pond for terminal lakes reclamation, water research, 44 (2010) 4601-4615.

[11] D. Park, E. Norouzi, C. Park, Experimental and Numerical Study of Water Distillation Performance of Small-Scale Direct Contact Membrane Distillation System, in: ASME 2017 International Mechanical Engineering Congress and Exposition, American Society of Mechanical Engineers, 2017, pp. V008T010A044-V008T010A044.

[12] K. Gethard, O. Sae-Khow, S. Mitra, Water desalination using carbon-nanotubeenhanced membrane distillation, ACS applied materials \& interfaces, 3 (2010) 110-114.

[13] A. Striolo, The mechanism of water diffusion in narrow carbon nanotubes, Nano letters, 6 (2006) 633-639.

[14] J.A. Thomas, A.J. McGaughey, Reassessing fast water transport through carbon nanotubes, Nano letters, 8 (2008) 2788-2793.

[15] A.K. An, E.-J. Lee, J. Guo, S. Jeong, J.-G. Lee, N. Ghaffour, Enhanced vapor transport in membrane distillation via functionalized carbon nanotubes anchored into electrospun nanofibres, Scientific reports, 7 (2017) 41562.

[16] L.F. Dumée, K. Sears, J. Schütz, N. Finn, C. Huynh, S. Hawkins, M. Duke, S. Gray, Characterization and evaluation of carbon nanotube Bucky-Paper membranes for direct contact membrane distillation, Journal of Membrane Science, 351 (2010) 36-43.

[17] S.M. Cooper, H.F. Chuang, M. Cinke, B.A. Cruden, M. Meyyappan, Gas permeability of a buckypaper membrane, Nano Letters, 3 (2003) 189-192.

[18] A. Munjiza, The combined finite-discrete element method, Wiley Online Library, 2004. 
[19] S. Plimpton, Fast parallel algorithms for short-range molecular dynamics, Journal of computational physics, 117 (1995) 1-19.

[20] M. Khayet, Membranes and theoretical modeling of membrane distillation: a review, Advances in colloid and interface science, 164 (2011) 56-88.

[21] J. Phattaranawik, R. Jiraratananon, A. Fane, C. Halim, Mass flux enhancement using spacer filled channels in direct contact membrane distillation, Journal of membrane Science, 187 (2001) 193-201.

[22] L. Martínez-Díez, M.I. Vazquez-Gonzalez, Temperature and concentration polarization in membrane distillation of aqueous salt solutions, Journal of membrane Science, 156 (1999) 265-273.

[23] R. Schofield, A. Fane, C. Fell, R. Macoun, Factors affecting flux in membrane distillation, Desalination, 77 (1990) 279-294.

[24] T.L. Bergman, F.P. Incropera, D.P. DeWitt, A.S. Lavine, Fundamentals of heat and mass transfer, John Wiley \& Sons, 2011.

[25] J. Phattaranawik, R. Jiraratananon, A. Fane, Effect of pore size distribution and air flux on mass transport in direct contact membrane distillation, Journal of membrane Science, 215 (2003) 75-85.

[26] T.-C. Chen, C.-D. Ho, H.-M. Yeh, Theoretical modeling and experimental analysis of direct contact membrane distillation, Journal of membrane Science, 330 (2009) 279-287.

[27] M.A.E.-R. Abu-Zeid, Y. Zhang, H. Dong, L. Zhang, H.-L. Chen, L. Hou, A comprehensive review of vacuum membrane distillation technique, Desalination, 356 (2015) 1-14. 
[28] E. Norouzi, M. Amidpour, Optimal thermodynamic and economic volume of a heat recovery steam generator by constructal design, International Communications in Heat and Mass Transfer, 39 (2012) 1286-1292.

[29] B. Sundén, R.M. Manglik, Plate heat exchangers: design, applications and performance, Wit Press, 2007.

[30] ANSYS ${ }^{\circledR}$, Academic research, release 18.0.

[31] D.C. Wilcox, Turbulence modeling for CFD, DCW industries La Canada, CA, 1998.

[32] T.L. Bergman, F.P. Incropera, Fundamentals of heat and mass transfer, John Wiley \& Sons, 2011.

[33] T. Uemura, K. Kotera, M. Henmi, H. Tomioka, Membrane technology in seawater desalination: History, recent developments and future prospects, Desalination and Water Treatment, 33 (2011) 283-288.

[34] M. Latorre, Environmental impact of brine disposal on Posidonia seagrasses, Desalination, 182 (2005) 517-524.

[35] M.A. Shannon, P.W. Bohn, M. Elimelech, J.G. Georgiadis, B.J. Marinas, A.M. Mayes, Science and technology for water purification in the coming decades, in: Nanoscience and technology: a collection of reviews from nature Journals, World Scientific, 2010, pp. 337346.

[36] Y. Guan, J. Li, F. Cheng, J. Zhao, X. Wang, Influence of salt concentration on DCMD performance for treatment of highly concentrated $\mathrm{NaCl}, \mathrm{KCl}, \mathrm{MgCl} 2$ and $\mathrm{MgSO} 4$ solutions, Desalination, 355 (2015) 110-117. 
[37] F. Edwie, T.-S. Chung, Development of hollow fiber membranes for water and salt recovery from highly concentrated brine via direct contact membrane distillation and crystallization, Journal of membrane science, 421 (2012) 111-123.

[38] A. Alkhudhiri, N. Darwish, N. Hilal, Membrane distillation: a comprehensive review, Desalination, 287 (2012) 2-18.

[39] M. Gryta, Desalination of thermally softened water by membrane distillation process, Desalination, 257 (2010) 30-35.

[40] J.A. Bush, J. Vanneste, T.Y. Cath, Membrane distillation for concentration of hypersaline brines from the Great Salt Lake: Effects of scaling and fouling on performance, efficiency, and salt rejection, Separation and Purification Technology, 170 (2016) 78-91. [41] X. Ji, E. Curcio, S. Al Obaidani, G. Di Profio, E. Fontananova, E. Drioli, Membrane distillation-crystallization of seawater reverse osmosis brines, Separation and Purification Technology, 71 (2010) 76-82.

[42] W. Wang, Y. Shi, C. Zhang, S. Hong, L. Shi, J. Chang, R. Li, Y. Jin, C. Ong, S. Zhuo, Simultaneous production of fresh water and electricity via multistage solar photovoltaic membrane distillation, Nature communications, 10 (2019) 1-9.

[43] C.M. Tun, A.G. Fane, J.T. Matheickal, R. Sheikholeslami, Membrane distillation crystallization of concentrated salts-flux and crystal formation, Journal of Membrane Science, 257 (2005) 144-155.

[44] N.R. Mene, Z. Murthy, Recovery of pure water and crystalline products from concentrated brine by using membrane distillation crystallization, Separation Science and Technology, 54 (2019) 396-408. 
[45] D. Lu, P. Li, W. Xiao, G. He, X. Jiang, Simultaneous recovery and crystallization control of saline organic wastewater by membrane distillation crystallization, AIChE Journal, 63 (2017) 2187-2197.

[46] R.A. Tufa, E. Curcio, E. Brauns, W. van Baak, E. Fontananova, G. Di Profio, Membrane distillation and reverse electrodialysis for near-zero liquid discharge and low energy seawater desalination, Journal of Membrane Science, 496 (2015) 325-333.

[47] J. Hermia, Constant pressure blocking filtration laws-application to power-law nonNewtonian fluids, Chem. Eng. Res. Des., 60 (1982) 183-187.

[48] M. Ramezanianpour, M. Sivakumar, An analytical flux decline model for membrane distillation, Desalination, 345 (2014) 1-12.

[49] S.L. Goren, The hydrodynamic force resisting the approach of a sphere to a plane permeable wall, Journal of Colloid and Interface Science, 69 (1979) 78-85.

[50] J.K. Holt, H.G. Park, Y. Wang, M. Stadermann, A.B. Artyukhin, C.P. Grigoropoulos, A. Noy, O. Bakajin, Fast mass transport through sub-2-nanometer carbon nanotubes, Science, 312 (2006) 1034-1037.

[51] M. Majumder, N. Chopra, R. Andrews, B.J. Hinds, Nanoscale hydrodynamics: enhanced flow in carbon nanotubes, Nature, 438 (2005) 44.

[52] S. Joseph, N. Aluru, Why are carbon nanotubes fast transporters of water?, Nano letters, 8 (2008) 452-458.

[53] C. Vega, J.L. Abascal, Simulating water with rigid non-polarizable models: a general perspective, Physical Chemistry Chemical Physics, 13 (2011) 19663-19688.

[54] F. Chen, P.E. Smith, Simulated surface tensions of common water models, in, AIP, 2007. 
[55] H. Berendsen, J. Grigera, T. Straatsma, The missing term in effective pair potentials, Journal of Physical Chemistry, 91 (1987) 6269-6271.

[56] J.-P. Ryckaert, G. Ciccotti, H.J. Berendsen, Numerical integration of the cartesian equations of motion of a system with constraints: molecular dynamics of n-alkanes, Journal of Computational Physics, 23 (1977) 327-341.

[57] B.-B. Wang, X.-D. Wang, Y.-Y. Duan, M. Chen, Molecular dynamics simulation on evaporation of water and aqueous droplets in the presence of electric field, International Journal of Heat and Mass Transfer, 73 (2014) 533-541.

[58] S. Koneshan, J.C. Rasaiah, R. Lynden-Bell, S. Lee, Solvent structure, dynamics, and ion mobility in aqueous solutions at 25 C, The Journal of Physical Chemistry B, 102 (1998) 4193-4204.

[59] O.N. Samoylova, E.I. Calixte, K.L. Shuford, Molecular dynamics simulations of ion transport in carbon nanotube channels, The Journal of Physical Chemistry C, 119 (2015) 1659-1666.

[60] R.W. Hockney, J.W. Eastwood, Computer simulation using particles, crc Press, 1988.

[61] V.P. Sokhan, D. Nicholson, N. Quirke, Fluid flow in nanopores: Accurate boundary conditions for carbon nanotubes, The Journal of chemical physics, 117 (2002) 8531-8539. [62] S. Gruener, P. Huber, Knudsen diffusion in silicon nanochannels, Physical review letters, 100 (2008) 064502.

[63] S. Jakobtorweihen, C. Lowe, F. Keil, B. Smit, Diffusion of chain molecules and mixtures in carbon nanotubes: The effect of host lattice flexibility and theory of diffusion in the Knudsen regime, The Journal of chemical physics, 127 (2007) 024904. 
[64] N. Bui, E.R. Meshot, S. Kim, J. Peña, P.W. Gibson, K.J. Wu, F. Fornasiero, Ultrabreathable and Protective Membranes with Sub-5 nm Carbon Nanotube Pores, Advanced Materials, 28 (2016) 5871-5877.

[65] B.K. Peterson, K.E. Gubbins, Phase transitions in a cylindrical pore: grand canonical Monte Carlo, mean field theory and the Kelvin equation, Mol. Phys., 62 (1987) 215-226.

[66] T. Werder, J.H. Walther, P. Koumoutsakos, Hydrodynamics of Carbon NanotubesContact Angle and Hydrophobic Hydration, in: Technical Proceedings of the 2002 International Conference on Computational Nanoscience and Nanotechnology, 2002.

[67] K.V. Agrawal, S. Shimizu, L.W. Drahushuk, D. Kilcoyne, M.S. Strano, Observation of extreme phase transition temperatures of water confined inside isolated carbon nanotubes, Nature nanotechnology, 12 (2017) 267.

[68] V.V. Chaban, O.V. Prezhdo, Water boiling inside carbon nanotubes: toward efficient drug release, ACS nano, 5 (2011) 5647-5655. 


\section{VITA}

Elnaz Norouzi was born in Tehran, Iran. After completing her schoolwork in 2004, Elnaz entered University of Tehran in Tehran, Iran. She received a Bachelor of Science with a major in Mechanical Engineering from University of Tehran in September 2008. She then attended Khajeh Nasir Toosi University of Technology and earned her master's degree in Mechanical Engineering (Energy Systems) in 2011. After four years of industrial and teaching experience, she started her $\mathrm{PhD}$ at University of Missouri in August 2015 with a major in Mechanical and Aerospace Engineering (MAE) and during her Ph.D. years, she has conducted research on multiscale phase change, heat and mass transfer in desalination and thermal management technologies. 